\title{
SARS-CoV-2 RBD antibodies that maximize breadth and resistance to escape
}

https://doi.org/10.1038/s41586-021-03807-6

Received: 29 March 2021

Accepted: 6 July 2021

Published online: 14 July 2021

Check for updates

\author{
Tyler N. Starr ${ }^{1,15}$, Nadine Czudnochowski ${ }^{2,15}$, Zhuoming Liu ${ }^{3,15}$, Fabrizia Zatta ${ }^{4}$, Young-Jun Park ${ }^{5}$, \\ Amin Addetia', Dora Pinto ${ }^{4}$, Martina Beltramello ${ }^{4}$, Patrick Hernandez ${ }^{2}$, Allison J. Greaney ${ }^{1,6}$, \\ Roberta Marzi ${ }^{4}$, William G. Glass ${ }^{7}$, Ivy Zhang ${ }^{7,8}$, Adam S. Dingens', John E. Bowen ${ }^{5}$, \\ M. Alejandra Tortorici ${ }^{5}$, Alexandra C. Walls ${ }^{5}$, Jason A. Wojcechowskyj ${ }^{2}$, Anna De Marco ${ }^{4}$, \\ Laura E. Rosen², Jiayi Zhou², Martin Montiel-Ruiz², Hannah Kaiser², Josh R. Dillen², \\ Heather Tucker ${ }^{2}$, Jessica Bassi ${ }^{4}$, Chiara Silacci-Fregni ${ }^{4}$, Michael P. Housley ${ }^{2}$, Julia di lulio ${ }^{2}$, \\ Gloria Lombardo ${ }^{4}$, Maria Agostini ${ }^{2}$, Nicole Sprugasci ${ }^{4}$, Katja Culap ${ }^{4}$, Stefano Jaconi ${ }^{4}$, \\ Marcel Meury ${ }^{2}$, Exequiel Dellota $\mathrm{Jr}^{2}$, Rana Abdelnabi ${ }^{9}$, Shi-Yan Caroline Foo ${ }^{9}$, \\ Elisabetta Cameroni ${ }^{4}$, Spencer Stumpf ${ }^{3}$, Tristan I. Croll ${ }^{10}$, Jay C. Nix ${ }^{11}$, Colin Havenar-Daughton ${ }^{2}$, \\ Luca Piccoli ${ }^{4}$, Fabio Benigni ${ }^{4}$, Johan Neyts ${ }^{9}$, Amalio Telenti ${ }^{2}$, Florian A. Lempp ${ }^{2}$, \\ Matteo S. Pizzuto ${ }^{4}$, John D. Chodera ${ }^{7}$, Christy M. Hebner ${ }^{2}$, Herbert W. Virgin ${ }^{2,12,13}$, \\ Sean P. J. Whelan ${ }^{3}$, David Veesler ${ }^{5}$, Davide Corti ${ }^{4 \bowtie}$, Jesse D. Bloom ${ }^{1,6,14 \bowtie}$ \& Gyorgy Snell $^{2 \bowtie}$
}

\begin{abstract}
An ideal therapeutic anti-SARS-CoV-2 antibody would resist viral escape ${ }^{1-3}$, have activity against diverse sarbecoviruses ${ }^{4-7}$, and be highly protective through viral neutralization $^{8-11}$ and effector functions ${ }^{12,13}$. Understanding how these properties relate to each other and vary across epitopes would aid the development of therapeutic antibodies and guide vaccine design. Here we comprehensively characterize escape, breadth and potency across a panel of SARS-CoV-2 antibodies targeting the receptor-binding domain (RBD). Despite a trade-off between in vitro neutralization potency and breadth of sarbecovirus binding, we identify neutralizing antibodies with exceptional sarbecovirus breadth and a corresponding resistance to SARS-CoV-2 escape. One of these antibodies, S2H97, binds with high affinity across all sarbecovirus clades to a cryptic epitope and prophylactically protects hamsters from viral challenge. Antibodies that target the angiotensin-converting enzyme 2 (ACE2) receptor-binding motif (RBM) typically have poor breadth and are readily escaped by mutations despite high neutralization potency. Nevertheless, we also characterize a potent $\mathrm{RBM}$ antibody $\left(\mathrm{S} 2 \mathrm{E} 12^{8}\right)$ with breadth across sarbecoviruses related to SARS-CoV-2 and a high barrier to viral escape. These data highlight principles underlying variation in escape, breadth and potency among antibodies that target the RBD, and identify epitopes and features to prioritize for therapeutic development against the current and potential future pandemics.
\end{abstract}

The most potently neutralizing antibodies to SARS-CoV-2-including those in clinical use ${ }^{14}$ and dominant in polyclonal sera ${ }^{15,16}$-target the spike RBD. Mutations in the RBD that reduce binding by antibodies have emerged among SARS-CoV-2 variants ${ }^{17-21}$, highlighting the need for antibodies and vaccines that are robust to viral escape. We have previously described $\mathrm{S} 309^{4}$, an antibody that exhibits potent effector functions and neutralizes all current SARS-CoV-2 variants ${ }^{22,23}$ and the divergent sarbecovirus SARS-CoV-1.S309 forms the basis for an antibody therapy (VIR-7831, recently renamed sotrovimab) that has received emergency use authorization from the US Food and Drug Administration for treatment of COVID-19 ${ }^{24}$. Longer term, antibodies with broad activity across sarbecoviruses would be useful to combat potential future spillovers ${ }^{6}$. These efforts would be aided by a systematic understanding of the relationships between antibody epitope, resistance to viral escape, and breadth of sarbecovirus cross-reactivity. Here we address this question by comprehensively characterizing a diverse panel of antibodies, including S309, using deep mutational scanning, pan-sarbecovirus binding assays, in vitro selection of viral escape, and biochemical and structural analyses.

${ }^{1}$ Basic Sciences Division, Fred Hutchinson Cancer Research Center, Seattle, WA, USA. ${ }^{2}$ Vir Biotechnology, San Francisco, CA, USA. ${ }^{3}$ Department of Molecular Microbiology, Washington University School of Medicine, St Louis, MO, USA. ${ }^{4}$ Humabs BioMed SA, a subsidiary of Vir Biotechnology, Bellinzona, Switzerland. ${ }^{5}$ Department of Biochemistry, University of Washington, Seattle, WA, USA. ${ }^{6}$ Department of Genome Sciences, University of Washington, Seattle, WA, USA. ${ }^{7}$ Computational and Systems Biology Program, Sloan Kettering Institute, Memorial Sloan Kettering Cancer Center, New York, NY, USA. ${ }^{8}$ Tri-Institutional PhD Program in Computational Biology and Medicine, Weill Cornell Graduate School of Medical Sciences, New York, NY, USA. ${ }^{9}$ Rega Institute for Medical Research, Laboratory of Virology and Chemotherapy, KU Leuven, Leuven, Belgium. ${ }^{10}$ Cambridge Institute for Medical Research, Department of Haematology,

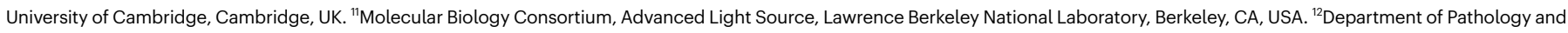
Immunology, Washington University School of Medicine, St Louis, MO, USA. ${ }^{13}$ Department of Internal Medicine, UT Southwestern Medical Center, Dallas, TX, USA. ${ }^{14}$ Howard Hughes Medical Institute, Seattle, WA, USA. ${ }^{15}$ These authors contributed equally: Tyler N. Starr, Nadine Czudnochowski, Zhuoming Liu. ${ }^{\bowtie e-m a i l: ~ d c o r t i @ v i r . b i o ; ~ j b l o o m @ f r e d h u t c h . o r g ; ~ g s n e l l @ v i r . b i o ~}$ 


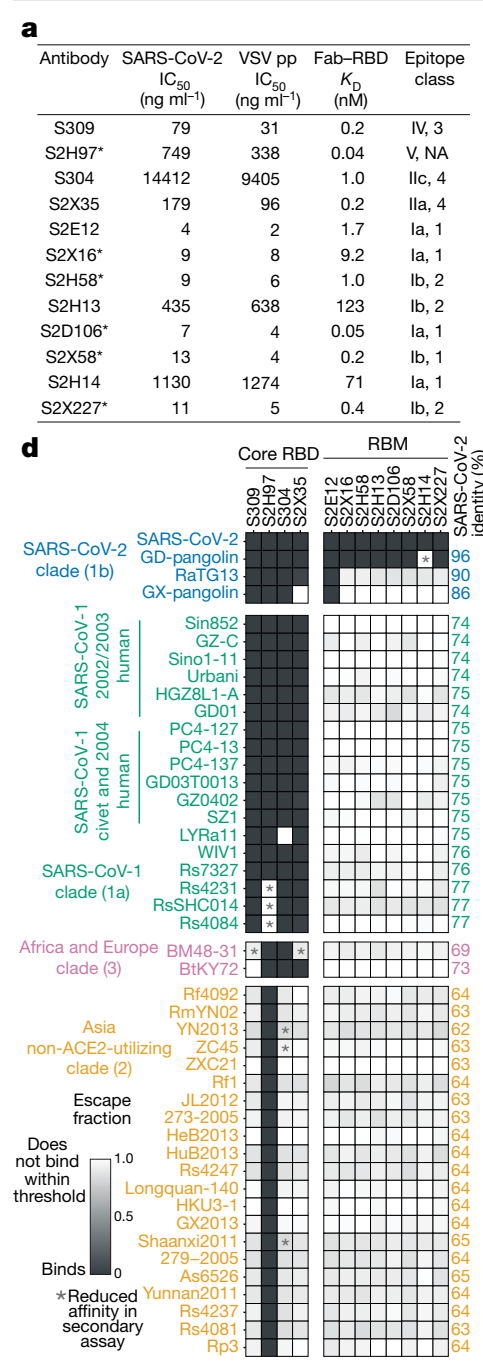

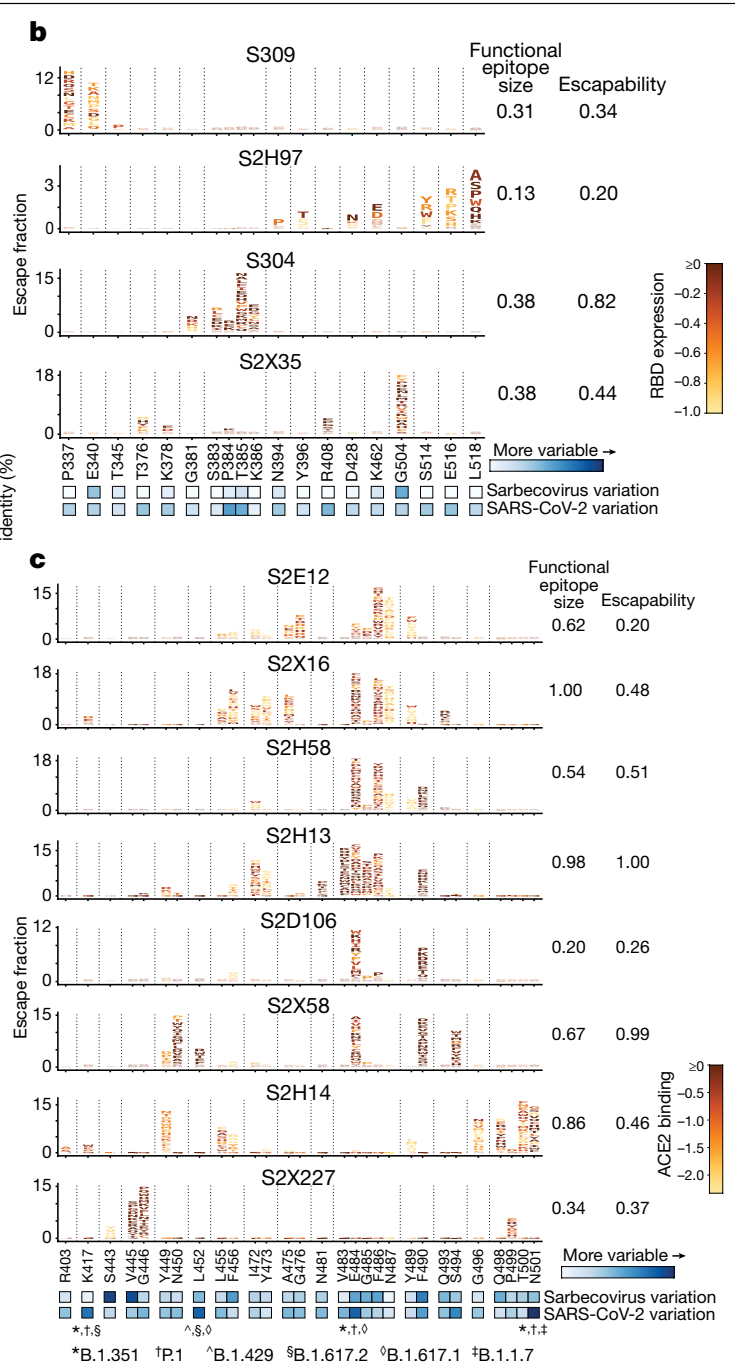

Fig. 1 Potency, escapability, and breadth of a panel of RBD antibodies.

a, SARS-CoV-2 neutralization potency (authentic virus $(n=3)$ and spike-pseudotyped VSV particles (VSV pp) ( $n=3$ to 8 ) on Vero E6 cells), Fab-RBD binding affinities measured by surface plasmon resonance (SPR) ( $n=2$ to 4$)$, and epitope classifications. Additional details in Extended Data Table 1. Asterisks indicate antibodies that are newly described in this paper. Epitope classes are described in refs. ${ }^{9,15}$. IC50, half-maximal inhibitory concentration; $K_{\mathrm{D}}$, dissociation constant; NA, not applicable. b, c, Left, deep mutational scanning maps of mutations that escape binding by antibodies targeting the core RBD (b) or the RBM (c). Letter height indicates the strength of the mutation's escape from antibody binding. Letters coloured by effect on folded RBD expression (b) or ACE2 binding affinity ${ }^{26}(\mathbf{c})$. Right, relative functional epitope size and escapability, scaling from 0 (no escape mutations) to 1 (largest epitope and most escapable antibody). Bottom, heat maps illustrate variability among sarbecoviruses or SARS-CoV-2 sequences. d, Antibody binding to a pan-sarbecovirus RBD panel. Heat map illustrates binding from fluorescence-activated cell sorting (FACS)-based selections (colour bar, bottom left). Asterisks indicate reduced-affinity binding in secondary binding assays (Extended Data Fig. 4a-f).

\section{Antibody potency, escapability and breadth}

We identified a panel of anti-SARS-CoV-2antibodies with distinct properties (Fig.1a,Extended Data Table1), including six antibodies newly described in thisstudy. These antibodies bind different epitopes within the RBM and the non-RBM 'core' of the RBD. The antibody panel spans a range of neutralization potencies and binding affinities (Fig. 1a, Extended Data Fig. 1a-c).

We used deep mutational scanning to map how all amino-acid mutations in the SARS-CoV-2 RBD affect binding by each antibody ${ }^{3}$ (Fig. 1b, c, Extended Data Fig. 2). Some antibodies have narrowly focused functional epitopes (the set of residues in which mutations abolish binding ${ }^{25}$ ), with binding-escape mutations at just a few key residues (for example, S309, S2D106), whereas others have wider functional epitopes (for example, S2H13; Fig. 1b, c). We previously measured how all RBD mutations affect folded RBD expression and ACE2 binding affinity ${ }^{26}$ (Fig. 1b, c, letter colours). We used the combined measures of how mutations affect antibody binding and RBD function to compute the 'escapability' of each antibody, which reflects the extent to which mutations that escape antibody binding are functionally tolerated (Fig. 1b, c, Extended Data Fig. 3a, b). We also investigated the sensitivity of each antibody to mutations among SARS-CoV-2 sequences reported in GISAID (Fig. 1b, c, heat map, Extended Data Fig. 3c), and found that some antibodies are more affected by natural SARS-CoV-2 mutations than others, including mutations found in SARS-CoV-2 variants of concern ${ }^{27-29}$ (Extended Data Fig. 1d).

We next extended our deep mutational scanning platform to measure binding of each antibody to a pan-sarbecovirus panel of 45 RBDs
(Fig. 1d, Extended Data Fig. 4a-f). The four antibodies that bind the core RBD exhibit cross-reactive binding to RBDs from SARS-CoV-1 and related ACE2-dependent bat sarbecoviruses, and from sarbecoviruses isolated in Europe and Africa. S304 and S2H97 antibodies also bind RBDs of the most divergent clade from Asia, which has an average of $64 \%$ amino acid identity with SARS-CoV-2. S2H97 exhibits notably tight binding to all RBDs tested (Fig. 1d, Extended Data Fig. 4f), making it, to our knowledge, the broadest pan-sarbecovirus RBD antibody described to date. Antibodies that bind epitopes within the RBM exhibit more limited cross-reactivity, typically binding only SARS-CoV-2 and the closely related GD-pangolin-CoV RBD. S2E12 stands out among the RBM antibodies we evaluated, as it also binds the RaTG13 and GX-pangolin-CoV RBDs, showing that even within the evolutionarily plastic $\mathrm{RBM}^{19,26}$ there are epitopes that enable greater breadth than others.

\section{The pan-sarbecovirus S2H97 antibody}

To understand the structural basis for cross-reactive sarbecovirus binding, we determined the structures of the $\mathrm{S} 2 \mathrm{H} 97$ antigen-binding fragment (Fab) (X-ray crystallography, 2.65 ̊̊ resolution), S2X35 Fab (X-ray crystallography, 1.83 Å resolution) and S2E12 Fab (X-ray crystallography, 2.95 Å resolution) bound to SARS-CoV-2 RBD (Fig. 2a, Extended Data Table 2). This panel of cross-reactive antibodies emphasizes the core RBD as a general target of broad antibody binding owing to its conservation among sarbecoviruses, reflected in the diverse core RBD 

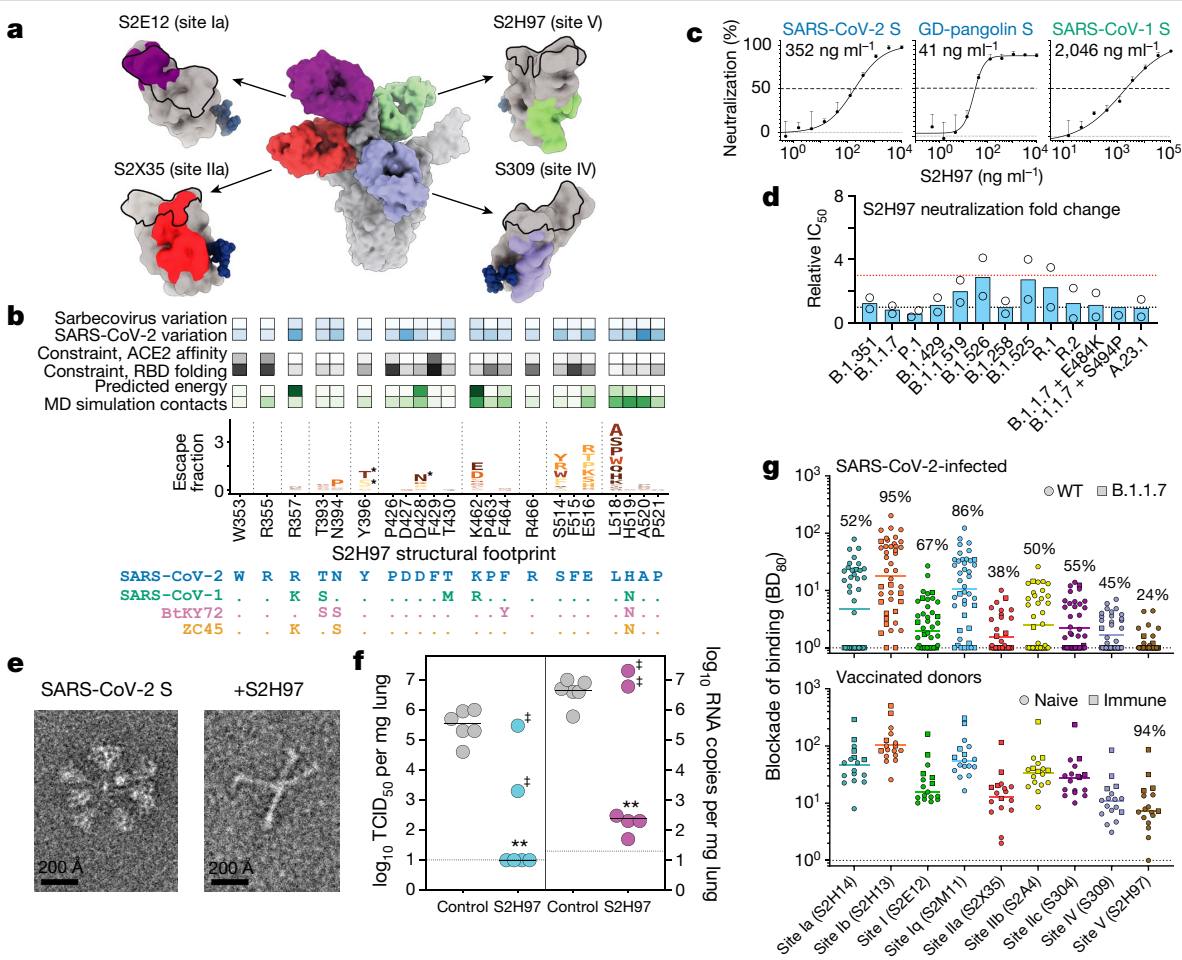

Fig. 2 | The pan-sarbecovirus S2H97 antibody. a, Composite model of the SARS-CoV-2 trimer with cross-reactive antibodies. Epitopes recognized by each Fab are shown as coloured surface and ACE2 footprint is shown as a black outline. b. Integrative features of the $\mathrm{S} 2 \mathrm{H} 97$ structural footprint (5 Å cut-off). Heat maps illustrate evolutionary variability (blue), functional constraint from previous deep mutational scans (grey) and energetic contribution to binding from the static crystal structure (predicted energy) or molecular dynamics (MD) simulation (green). Logo plot as in Fig. 1b. Asterisk, introduction of $N$-linked glycosylation motifs. c, S2H97 breadth of neutralization (spike-pseudotyped VSV on Vero E6 cells). Curves representative of at least two independent experiments. Data are mean \pm s.d. from three technical replicates. Geometric mean $\mathrm{IC}_{50}$ is shown.d, $\mathrm{S} 2 \mathrm{H} 97$ neutralization of SARS-CoV-2 variants (Extended Data Fig. 1d; spike-pseudotyped VSV on Vero E6 cells). Points represent individual measurements and bars show mean fold-change in neutralization potency relative to the Wuhan-1 strain with the D614G S mutation. e, Negative-stain electron microscopy imaging of native-like soluble prefusion S trimer alone (left) or incubated with S2H97 (right). Micrographs are representative of 51 (SARS-CoV-2 S) and $173(\mathrm{~S}+\mathrm{S} 2 \mathrm{H} 97$ ) micrographs. f, S2H97 prophylactic efficacy in Syrian hamsters. Median infectious virus titres (left, TCID50) and RNA levels (right) 4 days after SARS-CoV-2 challenge in hamsters prophylactically dosed with $25 \mathrm{mg} \mathrm{kg}^{-1} \mathrm{~S} 2 \mathrm{H} 97$ or isotype control. The double daggers indicate two hamsters with undetectable S2H97 levels (below $50 \mathrm{ng} \mathrm{ml}^{-1}$ ) at the time of viral challenge. ${ }^{* *} P=0.0048$ (virus titre) and $P=0.0048$ (RNA) vs control, two-sided Mann-Whitney test (hamsters with no detectable serum antibody excluded). g, Blockade of binding ${ }^{15}$ by sera from SARS-CoV-2-infected (top) or vaccinated (bottom) individuals. $\mathrm{BD}_{80}$ is the concentration that blocks $80 \%$ of binding. Percentages of samples with blockade above the lower detection limit are indicated. WT, wild type. surfaces targeted by the broadest of these antibodies (Fig. 2a, Extended Data Fig. 5a-g).

The exceptionally cross-reactive $\mathrm{S} 2 \mathrm{H} 97$ antibody targets a previously undescribed cryptic antigenic site, which we designated site $\mathrm{V}$ (Fig. 2a, b). S2H97 binding is facilitated by packing of the heavy chain complementarity-determining region 3 (CDR3) into an RBD crevice at the centre of the epitope, together with polar contacts with all three heavy chain CDRs and the light chain CDR2 (Extended Data Fig. 5f). Molecular dynamics simulation of the S2H97 Fab-RBD complex highlights the durability of many of these interactions (Fig. 2b). The surface bound by $\mathrm{S} 2 \mathrm{H} 97$ is constrained by the deleterious effects of mutations on folded RBD expression ${ }^{26}$ (Fig. 2b), and this constraint is probably enhanced by quaternary packing with the $\mathrm{N}$-terminal domain in the closed spike trimer (Extended Data Fig. 6a). Consistent with the conservation of the $\mathrm{S} 2 \mathrm{H} 97$ epitope, $\mathrm{S} 2 \mathrm{H} 97$ neutralizes diverse sarbecoviruses (Fig. 2c, Extended Data Fig. 4g) and SARS-CoV-2 variants (Fig. 2d).

To understand the evolution of $\mathrm{S} 2 \mathrm{H} 97$ breadth, we measured breadth of binding by its germline form, $\mathrm{S} 2 \mathrm{H} 97_{\mathrm{GL}}$, in which we reverted the 13 somatic mutations (Extended Data Fig. 4h, i). S2H97 ${ }_{\mathrm{GL}}$ bound all tested sarbecovirus RBDs and exhibited particularly high affinity for SARS-CoV-2-related RBDs. Somatic mutations enhanced affinity across all sarbecoviruses by two orders of magnitude. This general increase in affinity together with the conservative amino acid replacements among paratope residues suggests that framework mutations may contribute to a general improvement in $\mathrm{S} 2 \mathrm{H} 97$ binding affinity.

To characterize the mechanism of S2H97 neutralization, we determined a cryo-electron microscopy structure of $\mathrm{S} 2 \mathrm{H} 97$ bound to SARS-CoV-2S (Extended Data Fig. 5i-l, Extended Data Table 3). S2H97 binding requires extensive opening of the RBD to unmask its cognate epitope (Extended Data Fig. 6b), even more than is required to access the cryptic antigenic site II $^{15}$. Similar to other antibodies that only bind the open $\mathrm{RBD}^{30,31}, \mathrm{~S} 2 \mathrm{H} 97$ induces rapid and premature refolding of spike into the post-fusion state (Fig. 2e), promotes S1 shedding of cell-surface-expressed spike (Extended Data Fig. 6c), and induces a low level of syncytia formation among spike-expressing cells (Extended Data Fig. 6d). S2H97 does not interfere with ACE2 binding (Extended Data Fig. 6e). Like other non-ACE2-competitive antibodies ${ }^{31,32}$, S2H97 neutralization is attenuated in cells that overexpress ACE2 (Extended Data Fig. 6f). Consistent with its ability to neutralize spike-mediated viral entry, S2H97 inhibits spike-mediated cell-cell fusion (Extended Data Fig. 6g). Together, these experiments suggest that the mechanism of neutralization by $\mathrm{S} 2 \mathrm{H} 97$ involves receptor-independent conversion of $\mathrm{S}$ to the post-fusion state $^{30}$, thereby inhibiting ACE2-mediated cell entry.

Next, we determined the prophylactic efficacy of S2H97 in vivo using a Syrian hamster model of infection. We administered hamsters 
with $25 \mathrm{mg} \mathrm{kg}^{-1} \mathrm{~S} 2 \mathrm{H} 972$ days before intranasal challenge with SARS-CoV-2 and assessed viral RNA load and infectious viral titres in the lungs 4 days after infection. S2H97 prophylaxis reduced the number of RNA copies by more than 10,000-fold relative to control in the four hamsters that had detectable circulating antibody levels at the time of challenge, and reduced infectious viral titres to the lower detection limit in these hamsters (Fig. 2f). The two hamsters not exhibiting a reduction in viral load had circulating $\mathrm{S} 2 \mathrm{H} 97 \mathrm{lev}$ els below the limit of quantification $\left(50 \mathrm{ng} \mathrm{ml}^{-1}\right)$ at the time of viral challenge (Extended Data Fig. 6h), which may reflect a failure in the intraperitoneal administration procedure. Therefore, S2H97 demonstrates that antibodies to the newly identified antigenic site $\mathrm{V}$ can be protective in vivo.

Finally, we performed serum blockade of binding experiments ${ }^{15}$, demonstrating that antibodies competing with $\mathrm{S} 2 \mathrm{H} 97$ binding are rare in infection- and vaccine-elicited sera (Fig. 2g). This sub-dominance of antigenic site $V$ may be explained by the inaccessibility of the epitope as illustrated in the cryoEM structure. However, the protective nature and exceptional breadth of $\mathrm{S} 2 \mathrm{H} 97$ suggests that updated immunogen designs, such as those based on the $\mathrm{RBD}^{33-35}$, could unmask antigenic site $\mathrm{V}$ to better elicit S2H97-like antibodies.

\section{Breadth and escapability among RBM epitopes}

Our survey reveals variation in the escapability and breadth of antibodies that target the RBM (Fig. 1c, d), which is immunodominant (Fig. 2g) but variable over sarbecovirus and SARS-CoV-2 evolution. We performed in vitro selection experiments to identify spike-expressing vesicular stomatitis virus (VSV) mutants that emerge in the presence of each of seven monoclonal antibodies (Fig. 3a, Extended Data Fig. 7a, b) to further understand escape from these antibodies.

Many RBM antibodies such as S2X58 and S2D106 select mutations present in SARS-CoV-2 variants of concern (for example, L452R and $\mathrm{E} 484 \mathrm{~K})^{27-29}$. By contrast, S2E12 selects viral mutants at sites that do not exhibit substantial variation among circulating SARS-CoV-2, and S2E12 correspondingly neutralizes a diverse panel of SARS-CoV-2 variants ${ }^{17}$ (Fig. 3b). S2E12 is also unique in its breadth among RBM antibodies (Fig. 1d), neutralizing VSV pseudotyped with each of the four SARS-CoV-2 clade sarbecovirus spikes (Fig. 3c, Extended Data Fig. 4j). As with S2H97, somatic mutations in S2E12 enhanced affinity across sarbecoviruses, though the increase in affinity was more modest than for S2H97 (Extended Data Fig. 4k, l).

Conservation of the S2E12 epitope among SARS-CoV-2 variants could reflect the relative rarity of S2E12-like antibodies in polyclonal sera leading to little antigenic pressure at these sites (Fig. 2g), together with functional constraint in the S2E12 epitope (escapability being the lowest for S2E12 and S2H97 among the 12 antibodies evaluated). Indeed, the strong antibody-escape mutations that emerged in S2E12 viral escape selections decrease ACE2 binding affinity ${ }^{26}$ (Fig. 3a) and reduce replicative fitness in a bulk competition experiment between spike-expressing VSV variants passaged in the absence of antibody (Fig. 3d).

To understand the structural basis for the unique breadth and robustness of S2E12 to escape, we compared its structure to that of S2D106 Fab (cryoEM, 4.0 Å resolution local refinement) bound to SARS-CoV-2 RBD (Fig. 3e, f, Extended Data Fig. 5g, h, m-p, Extended Data Tables 2, 3). We also integrated evolutionary, functional, and structural details for the sites in each antibody's structural footprint (Fig. 3g, h). S2E12 and S2D106 bind the receptor-binding ridge, with 8 residues shared between their footprints. S2E12 binding is oriented towards extensive packing of the ACE2-contact residue $F 486_{\mathrm{RBD}}$ within a cavity lined by aromatic residues at the antibody light-heavy-chain interface (Fig. 3e, Extended Data Fig. 5g), as was seen with the homologous antibody COV2-2196 ${ }^{36}$. Sites within the S2E12 footprint that exhibit less functional constraint (for example, E484 and S477) are located at the periphery of the interface, explaining the robustness of S2E12 towards SARS-CoV-2 variants (Fig. 3b, g). This structural interface also explains the breadth of S2E12 towards RaTG13 and GX-pangolin-CoV (Fig. 1d), as the F486L mutation present in these sarbecoviruses retains the central hydrophobic packing.

In contrast to S2E12, S2D106 binding is centred on residue $\mathrm{E} 484_{\mathrm{RBD}}$ which may form a salt bridge with $\mathrm{R} 96_{\mathrm{LC}}$, in addition to nonpolar contacts between $\mathrm{F} 490_{\mathrm{RBD}}$ and residues in the heavy chain CDR2 (Fig. 3f, Extended Data Fig. $5 \mathrm{~h}$ ). Although the long heavy chain CDR3 packs intimately across the surface of the RBD, there are no crucial CDRH3RBD contacts that are sensitive to mutation. S2D106 escape is therefore highly focused on E484 and F490, which are functionally tolerant and exhibit variation among SARS-CoV-2 sequences (Fig. $3 \mathrm{~h}$ ). This comparison between S2E12 and S2D106 highlights how small differences in the RBD-antibody interface affect the breadth and robustness of each antibody to viral escape.

\section{The landscape of RBD epitopes}

Last, we examined how escapability, breadth, and neutralization potency relate to one another and to the RBD epitope. We used our binding-escape maps (Fig. 1b, c), together with comparable maps published for other RBD antibodies ${ }^{3,20,21,36,37}$, to project antibodies into a two-dimensional space on the basis of similarities in sites of binding-escape mutations (Fig. 4a).

We annotated our projection of epitope space using antibody properties such as in vitro neutralization potency, breadth and escapability (Fig. 4b-d, Extended Data Fig. 7c, d). The most potently neutralizing antibodies (for example, S2E12 and S2D106) bind epitopes in the RBM, whereas antibodies targeting the core RBD are less potently neutralizing (Fig. 4b). Of note, RBD antibodies can protect in vivo through other mechanisms beyond neutralization ${ }^{12,13,22}$. Antibodies with broad sarbecovirus binding target the core RBD (Fig. 4c). Our panel therefore extends previous observations $s^{4,5,32,38}$ to highlight a general trade-off between sarbecovirus breadth and potency of SARS-CoV-2 neutralization (Fig. 4e). Nonetheless, some cross-reactive antibodies exhibit intermediate in vitro neutralization potency (for example, S309 and S2X $259^{37}$ ), and the highly potent RBM-directed antibody S2E12 exhibits modest breadth, highlighting the existence of antibodies that balance neutralization potency and breadth.

The size of an antibody's functional epitope (Fig. 1b, c) is not strongly influenced by the structural location of the epitope (Extended Data Fig. 7c)-instead, narrower functional epitopes are associated with higher Fab-RBD binding affinity (Fig. 4f). However, an antibody's escapability, which integrates how escape mutations affect RBD folding and ACE2 affinity, is influenced by variation in these functional constraints across the RBD structure. For example, antibodies that cluster with S2E12 exhibit lower escapability (Extended Data Fig. 7c) and frequency of natural SARS-CoV-2 escape mutants (Fig. 4d). As highlighted by S2E12 and S2H97 above, antibodies with even a modest degree of sarbecovirus breadth show a greatly reduced frequency of escape mutations among circulating SARS-CoV-2 variants (Fig. 4g).

\section{Principles for antibody and vaccine development}

Ongoing SARS-CoV-2 evolution ${ }^{19,27-29}$, long-term antigenic evolution of other human coronaviruses ${ }^{39,40}$ and the spillover potential of diverse sarbecovirus lineages ${ }^{6,7}$ indicate the importance of developing antibodies and vaccines that are robust to viral evolution. In this work, we identify antibody and epitope features that can guide this process. Although in vitro neutralization potency is often prioritized for lead selection, our results suggest that this will bias antibodies towards RBM epitopes, many of which are poorly conserved in the 

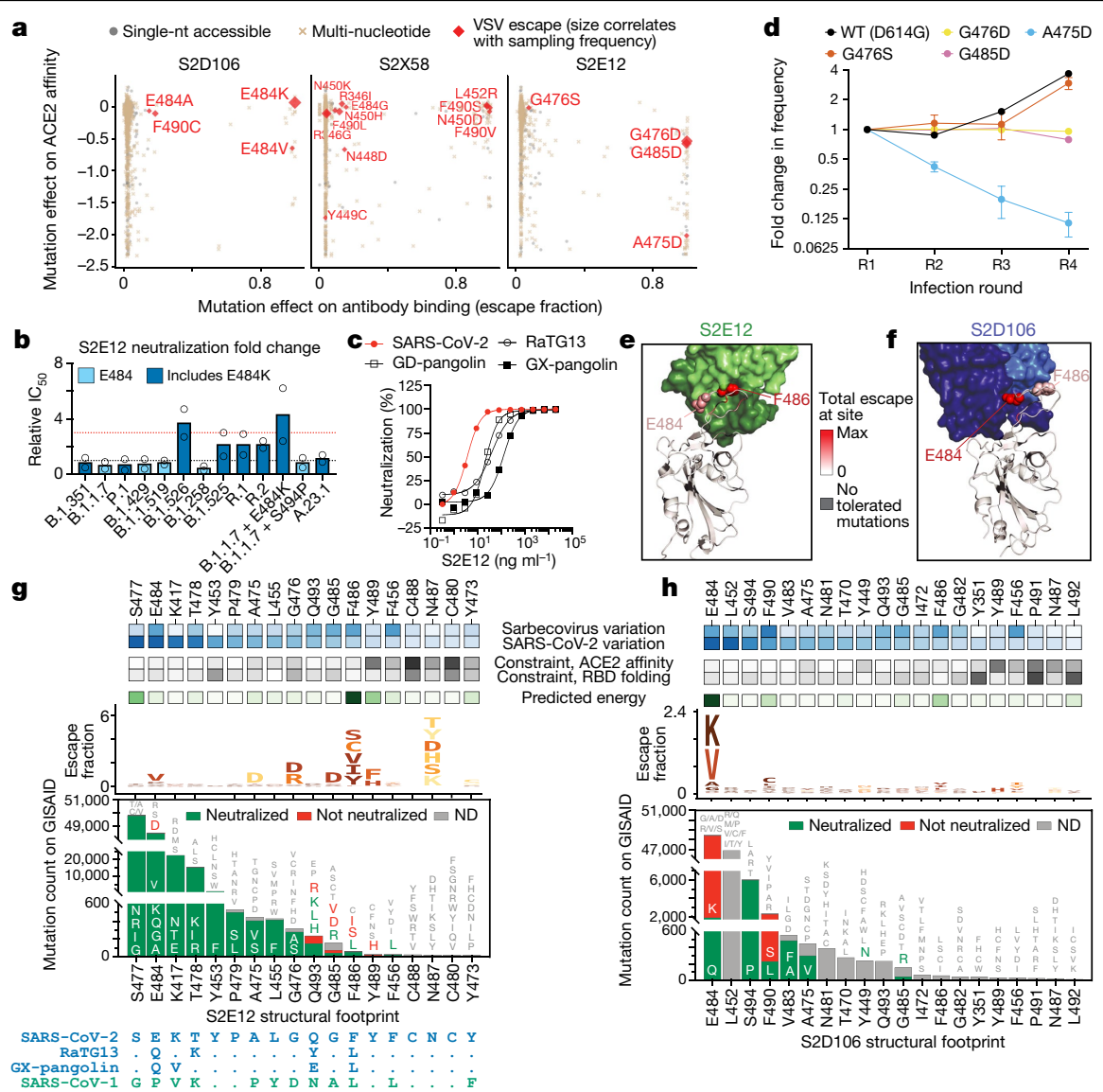

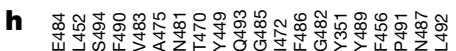
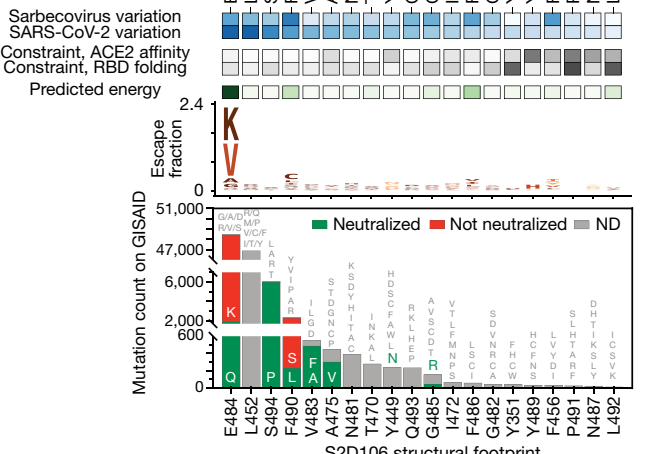

Fig. 3 | Breadth and escapability among RBM antibodies. a, Escape mutations in spike-expressing VSV passaged in the presence of monoclonal antibody. Plot shows effects of indicated mutations on antibody ( $x$-axis) and ACE2 ( $y$-axis) binding ${ }^{26}$. Mutations are classed according to whether they are accessible by single mutation from the Wuhan-1 strain (single-nt accessible) and those that require multi-nucleotide mutations. b, Neutralization of SARS-CoV-2 variants by S2E12 (spike-pseudotyped VSV on Vero E6 cells), relative to Wuhan-1 D614G, as in Fig. 2d.c, S2E12 breadth of neutralization (spike-pseudotyped VSV on 293T-ACE2 cells). Points represent mean of biological duplicates. d, Replicative fitness of S2E12 escape mutations identified in a on Vero E6 cells. Data are mean \pm s.e.m. from triplicate experiments. e, f, Structures of S2E12 Fab (e) and S2D106 Fab (f) bound to
SARS-CoV-2 RBD. RBD sites coloured by escape (colour bar, centre). The E484 side chain is included for visualization purposes only but was not included in the final S2D106-bound structure owing to weak density. Max, maximum. $\mathbf{g}$, h, Integrative features of the structural footprints (5 ̊ cut-off) of S2E12 (g) and S2D106 (h). Sites are ordered by the frequency of observed mutants among SARS-CoV-2 sequences on GISAID. Heat maps as in Fig. 2b. Logo plots as in Fig. 1c, but showing only amino acid mutations accessible via single-nucleotide mutation from Wuhan-1 strain for comparison with a. Bar plots illustrate frequency of SARS-CoV-2 mutants and their validated effects on antibody neutralization (spike-pseudotyped VSV on Vero E6 cells). Red indicates greater than tenfold increase in $\mathrm{IC}_{50}$ due to mutation. ND, not determined. short-term evolution of SARS-CoV- $2^{19}$ and the long-term evolution of sarbecoviruses ${ }^{7}$. Our results suggest that additional prioritization of high-affinity binding and at least a moderate degree of sarbecovirus breadth will yield antibodies with improved resistance to viral escape $^{4,5}$.

A long-term goal is to develop antibodies and vaccines that cross-react with distant sarbecovirus lineages capable of zoonotic spillover. We have identified a cryptic epitope capable of eliciting pan-sarbecovirus immunity, represented by S2H97. Though S2H97-like antibodies are rare in polyclonal sera, the protective capacity and exceptional breadth of $\mathrm{S} 2 \mathrm{H} 97$ indicates that pan-sarbecovirus vaccines could seek to improve responses to this epitope by unmasking this and other cryptic broadly neutralizing epitopes ${ }^{5,37,41}$. Broader cross-reactivity among betacoronavirus lineages including MERS and OC 43 has been reported for antibodies that bind the spike S2 domain ${ }^{32,38,42}$. Though S2H97 breadth does not extend beyond sarbecoviruses, its discovery expands our view of what can be achieved via a potent RBD-directed antibody response.

The global emergence of variants of concern (VOC) has been an important feature of the pandemic ${ }^{27-29}$. Mutations in VOC occur in immunodominant RBM epitopes (for example, residues E484, K417 and L452) and influence binding by polyclonal serum and some therapeutic antibodies ${ }^{17-21}$. We cannot predict which mutations will next rise to prominence as SARS-CoV-2 continues to evolve, but it seems likely that they will include additional RBM mutations that affect recognition by infection- and vaccine-elicited antibodies ${ }^{1,2,15,16,19}$. Therefore, antibody discovery efforts focused on breadth ${ }^{4,5}$, aided by high-resolution differentiation among antibody epitopes as generated here, can inform the development of antibody and vaccine countermeasures with greater robustness to immune escape in the current SARS-CoV-2 pandemic and utility for potential future sarbecovirus spillovers.

\section{Online content}

Any methods, additional references, Nature Research reporting summaries, source data, extended data, supplementary information, acknowledgements, peer review information; details of author contributions and competing interests; and statements of data and code availability are available at https://doi.org/10.1038/s41586-021-03807-6. 
a

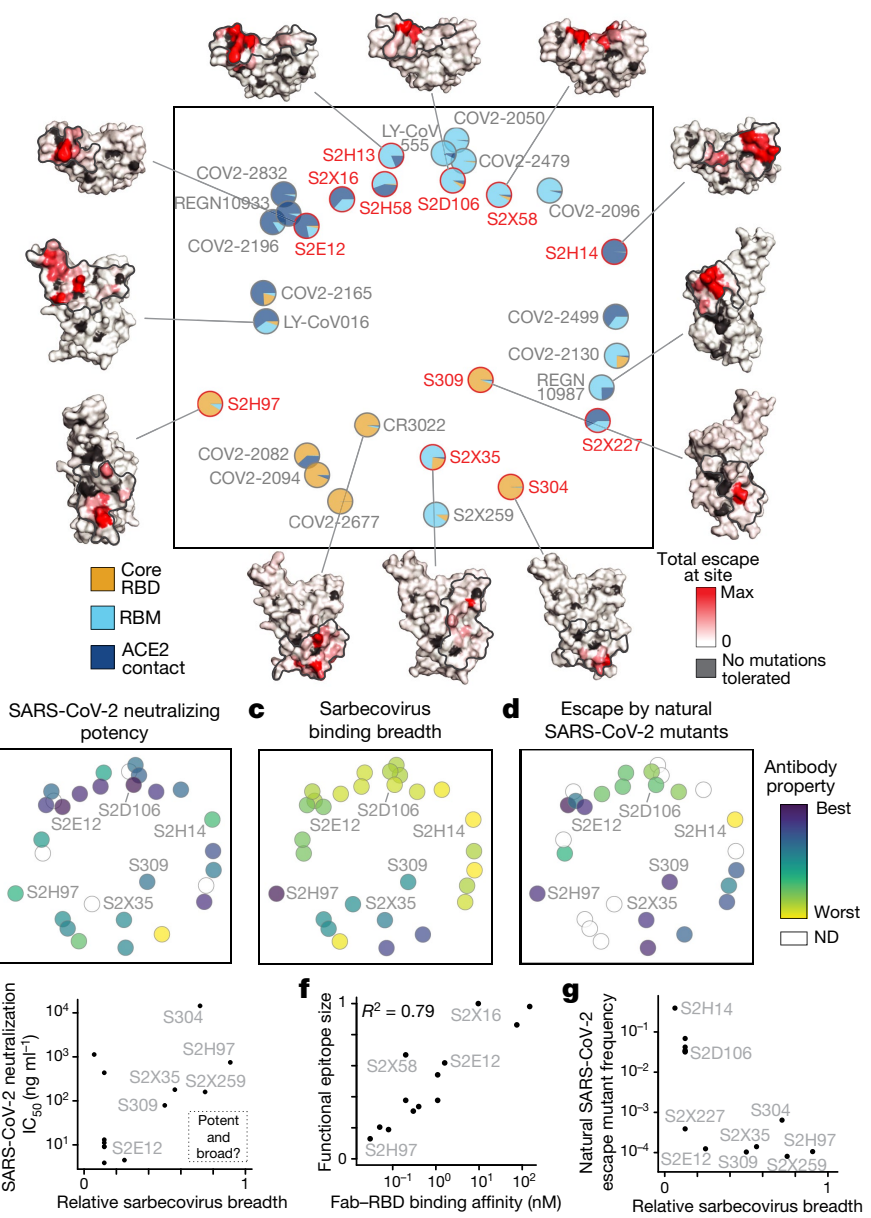

Fig. 4 | Antibody epitope, potency, breadth and escapability.

a, Multidimensional scaling projection of similarities in antibody binding-escape maps from the present (red) and previous (grey) studies. Pie charts illustrate the RBD subdomains where mutations confer escape (key, bottom left: RBM, residues 437-508; ACE2 contact, 4 Å cutoff). Structural projections of escape arrayed around the perimeter (colour bar, bottom right), with grey outlines tracing structural footprints. b-d, Projected epitope space from a annotated by antibody properties: SARS-CoV-2-neutralizing potency (b), sarbecovirus-binding breadth (c) and escape by natural SARS-CoV-2 mutants (d). For each property, antibodies are coloured such that purple reflects the most desirable antibody (colour bar, right): most potent neutralization ( $\log _{10}$ scale), highest breadth, and lowest natural frequency of escape mutants ( $\log _{10}$ scale).e, Relationship between SARS-CoV- 2 neutralization potency and sarbecovirus breadth for antibodies described in this work and in a parallel study $^{37}$ (S2X259).f, Relationship between functional epitope size and SARS-CoV-2 RBD binding affinity. $g$, Relationship between natural SARS-CoV-2 escape mutant frequency (Extended Data Fig. 3c) and sarbecovirus breadth.

1. Liu, Z. et al. Identification of SARS-CoV-2 spike mutations that attenuate monoclonal and serum antibody neutralization. Cell Host Microbe 29, 477-488.e4 (2021).

2. Weisblum, Y. et al. Escape from neutralizing antibodies by SARS-CoV-2 spike protein variants. eLife 9, e61312 (2020)

3. Greaney, A. J. et al. Complete mapping of mutations to the SARS-CoV-2 spike receptor-binding domain that escape antibody recognition. Cell Host Microbe 29, 44-57. e9 (2021).

4. Pinto, D. et al. Cross-neutralization of SARS-CoV-2 by a human monoclonal SARS-CoV antibody. Nature 583, 290-295 (2020).

5. Rappazzo, C. G. et al. Broad and potent activity against SARS-like viruses by an engineered human monoclonal antibody. Science 371, 823-829 (2021).

6. Menachery, V. D. et al. A SARS-like cluster of circulating bat coronaviruses shows potential for human emergence. Nat. Med. 21, 1508-1513 (2015).

7. Letko, M., Marzi, A. \& Munster, V. Functional assessment of cell entry and receptor usage for SARS-CoV-2 and other lineage B betacoronaviruses. Nat. Microbiol. 5, 562-569 (2020)
8. Tortorici, M. A. et al. Ultrapotent human antibodies protect against SARS-CoV-2 challenge via multiple mechanisms. Science 370, 950-957 (2020).

9. Barnes, C. O. et al. SARS-CoV-2 neutralizing antibody structures inform therapeutic strategies. Nature 588, 682-687 (2020).

10. Zost, S. J. et al. Potently neutralizing and protective human antibodies against SARS-CoV-2. Nature 584, 443-449 (2020).

11. Hassan, A. O. et al. A SARS-CoV-2 infection model in mice demonstrates protection by neutralizing antibodies. Cell 182, 744-753.e4 (2020).

12. Winkler, E. S. et al. Human neutralizing antibodies against SARS-CoV-2 require intact Fc effector functions for optimal therapeutic protection. Cell 184, 1804-1820.e16 (2021).

13. Schäfer, A. et al. Antibody potency, effector function, and combinations in protection and therapy for SARS-CoV-2 infection in vivo. J. Exp. Med. 218, e20201993 (2021).

14. Corti, D., Purcell, L. A., Snell, G. \& Veesler, D. Tackling COVID-19 with neutralizing monoclonal antibodies. Cell 184, 3086-3108 (2021).

15. Piccoli, L. et al. Mapping neutralizing and immunodominant sites on the SARS-CoV-2 spike receptor-binding domain by structure-guided high-resolution serology. Cell 183 1024-1042.e21 (2020).

16. Greaney, A. J. et al. Comprehensive mapping of mutations in the SARS-CoV-2 receptor-binding domain that affect recognition by polyclonal human plasma antibodies. Cell Host Microbe 29, 463-476.e6 (2021).

17. Chen, R. E. et al. Resistance of SARS-CoV-2 variants to neutralization by monoclonal and serum-derived polyclonal antibodies. Nat. Med. 27, 717-726 (2021).

18. Wang, P. et al. Antibody resistance of SARS-CoV-2 variants B.1.351 and B.1.1.7. Nature $\mathbf{5 9 3}$ 130-135 (2021).

19. Thomson, E. C. et al. Circulating SARS-CoV-2 spike N439K variants maintain fitness while evading antibody-mediated immunity. Cell 184, 1171-1187.e20 (2021).

20. Starr, T. N. et al. Prospective mapping of viral mutations that escape antibodies used to treat COVID-19. Science 371, 850-854 (2021).

21. Starr, T. N., Greaney, A. J., Dingens, A. S. \& Bloom, J. D. Complete map of SARS-CoV-2 RBD mutations that escape the monoclonal antibody LY-CoV555 and its cocktail with LY-CoV016. Cell Rep. Med. 2, 100255 (2021).

22. Cathcart, A. L. et al. The dual function monoclonal antibodies VIR-7831 and VIR-7832 demonstrate potent in vitro and in vivo activity against SARS-CoV-2. Preprint at https:// doi.org/10.1101/2021.03.09.434607 (2021).

23. McCallum, M. et al. SARS-CoV-2 immune evasion by the B.1.427/B.1.429 variant of concern. Science https://doi.org/10.1126/science.abi7994 (2021).

24. Gupta, A. et al. Early Covid-19 treatment with SARS-CoV-2 neutralizing antibody sotrovimab. Preprint at https://doi.org/10.1101/2021.05.27.21257096 (2021).

25. Dingens, A. S., Arenz, D., Weight, H., Overbaugh, J. \& Bloom, J. D. An antigenic atlas of HIV-1 escape from broadly neutralizing antibodies distinguishes functional and structural epitopes. Immunity 50, 520-532.e3 (2019).

26. Starr, T. N. et al. Deep mutational scanning of SARS-CoV-2 receptor binding domain reveals constraints on folding and ACE2 binding. Cell 182, 1295-1310.e20 (2020)

27. Faria, N. R. et al. Genomics and epidemiology of the P.1 SARS-CoV-2 lineage in Manaus, Brazil. Science 372, 815-821 (2021).

28. Tegally, H. et al. Detection of a SARS-CoV-2 variant of concern in South Africa. Nature 592, 438-443 (2021)

29. Zhang, W. et al. Emergence of a novel SARS-CoV-2 variant in southern California. J. Am. Med. Assoc. 325, 1324-1326 (2021).

30. Walls, A. C. et al. Unexpected receptor functional mimicry elucidates activation of coronavirus fusion. Cell 176, 1026-1039.e15 (2019).

31. Lempp, F. A. et al. Membrane lectins enhance SARS-CoV-2 infection and influence the neutralizing activity of different classes of antibodies. Preprint at https://doi. org/10.1101/2021.04.03.438258 (2021).

32. Pinto, D. et al. A human antibody that broadly neutralizes betacoronaviruses protects against SARS-CoV-2 by blocking the fusion machinery. Preprint at https://doi. org/10.1101/2021.05.09.442808 (2021).

33. Walls, A. C. et al. Elicitation of potent neutralizing antibody responses by designed protein nanoparticle vaccines for SARS-CoV-2. Cell 183, 1367-1382.e17 (2020).

34. Cohen, A. A. et al. Mosaic nanoparticles elicit cross-reactive immune responses to zoonotic coronaviruses in mice. Science 371, 735-741 (2021).

35. Saunders, K. O. et al. Neutralizing antibody vaccine for pandemic and pre-emergent coronaviruses. Nature 594, 553-559 (2021).

36. Dong, J. et al. Genetic and structural basis for recognition of SARS-CoV-2 spike protein by a two-antibody cocktail. Preprint at https://doi.org/10.1101/2021.01.27.428529 (2021).

37. Tortorici, M. A. et al. Broad sarbecovirus neutralization by a human monoclonal antibody Nature https://doi.org/10.1038/s41586-021-03817-4 (2021).

38. Sauer, M. M. et al. Structural basis for broad coronavirus neutralization. Nat. Struct. Mol. Biol. 28, 478-486 (2021).

39. Kistler, K. E. \& Bedford, T. Evidence for adaptive evolution in the receptor-binding domain of seasonal coronaviruses OC43 and 229e. eLife 10, e64509 (2021).

40. Eguia, R. T. et al. A human coronavirus evolves antigenically to escape antibody immunity. PLoS Pathog. 17, e1009453 (2021).

41. Walls, A. C. et al. Elicitation of broadly protective sarbecovirus immunity by receptor-binding domain nanoparticle vaccines. Preprint at https://doi.org/10.1101/ 2021.03.15.435528 (2021)

42. Zhou, P. et al. A protective broadly cross-reactive human antibody defines a conserved site of vulnerability on beta-coronavirus spikes. Preprint at https://doi.org/10.1101/ 2021.03.30.437769 (2021).

Publisher's note Springer Nature remains neutral with regard to jurisdictional claims in published maps and institutional affiliations.

(c) The Author(s), under exclusive licence to Springer Nature Limited 2021 


\section{Methods}

\section{Mammalian cell lines}

Cell lines were received from ATCC (Vero E6, Vero, BHK-21, CHO-K1, HEK293T/17), Takara (Lenti-X 293T) and Thermo Fisher Scientific (ExpiCHO-S, Expi293F and Freestyle 293-F). MA104 cells were a gift from H. B. Greenberg (Stanford School of Medicine). The 293T-ACE2 cells are described in references ${ }^{31,43}$. Vero and MA104 cell lines tested negative for mycoplasma contamination. Other cell lines were not tested. No authentication was performed beyond manufacturer standards.

\section{Isolation of peripheral blood mononuclear cells, plasma and sera}

Samples from three individuals who had recovered from SARS-CoV-2, designated as donors S2H (age 36, male), S2D (age 70, male) and S2X (age 52, male) were obtained under study protocols approved by the local Institutional Review Board (Canton Ticino Ethics Committee, Switzerland). All donors provided written informed consent for the use of blood and blood components (such as peripheral blood mononuclear cells (PBMCs), sera or plasma). Blood drawn from donor S2X was obtained at day 48 (S2X16, S2X35 and S2X58 antibodies) and 75 (S2X227) after symptoms onset. Blood from donor S2H was obtained at day 17 (S2H13 and S2H14), day 45 (S2H58) and day 81 (S2H97) after symptoms onset. Blood from donor S2D was obtained at day 98 (S2D106) after symptoms onset.

PBMCs were isolated from blood draw performed using tubes pre-filled with heparin, followed by Ficoll density gradient centrifugation. PBMCs were either used fresh for SARS-CoV-2 Spike protein-specific memory B cell sorting or stored in liquid nitrogen for later use. Sera were obtained from blood collected using tubes containing clot activator, followed by centrifugation and storage at $-80^{\circ} \mathrm{C}$.

Sera for blockade of binding serological assays were obtained from 3 cohorts of SARS-CoV-2 convalescent (average age 52, range $25-78,55 \%$ male) or vaccinated (average age 49 , range $28-69,65 \%$ male) individuals under study protocols approved by the local Institutional Review Boards (Canton Ticino Ethics Committee, Switzerland, the Ethical Committee of Luigi Sacco Hospital, Milan, Italy, and WCG North America, Princeton, NJ, USA). All donors provided written informed consent for the use of blood and blood components (such as PBMCs, sera or plasma) and were recruited at hospitals or as outpatients.

\section{B-cell isolation and recombinant monoclonal antibody production}

The discovery and initial characterization of six of the antibodies in our panel has been previously reported (S309 and S304, refs. ${ }^{4,15}$; S2X35, S2H13 and S2H14, ref. $^{15}$; and S2E12, ref. ${ }^{8}$ ), and the other six (S2H97, S2X16, S2H58, S2D106, S2X58 and S2X227) are described for the first time in this article. Starting from freshly isolated PBMCs or upon cell thawing, B cells were enriched by staining with CD19 PE-Cy7 (BD Bioscience 557835, 1:50) and incubation with anti-PE MicroBeads (Miltenyi Biotec 130-048-801, 1:100), followed by positive selection using LS columns (Miltenyi Biotec). Enriched B cells were stained with anti-IgM (BioLegend 314508, 1:20), anti-IgD (BD Bioscience 555779, 1:40), anti-CD14 (BD Bioscience 562691, 1:50) and anti-IgA (Southern Biotech 2050-09, 1:400), all PE labelled, and prefusion SARS-CoV-2 S with a biotinylated Avi-tag (in house produced) conjugated to Streptavidin-Alexa-Fluor 647 (Life Technologies S21374, 1:40). SARS-CoV-2 $\mathrm{S}$-specific $\operatorname{IgG}^{+}$memory B cells were sorted by flow cytometry via gating for PE negative and Alexa-Fluor 647 positive cells. Cells were cultured for the screening of positive supernatants. Antibody VH and VL sequences were obtained by PCR with reverse transcription (RT-PCR) and monoclonal antibodies were expressed as recombinant human Fab fragment or as IgG1 (G1m3 allotype) carrying the half-life-extending $\mathrm{M} 428 \mathrm{~L} / \mathrm{N} 434 \mathrm{~S}$ (LS) mutation in the Fc region. ExpiCHO-S cells (Thermo Fisher Scientific) were transiently transfected with heavy and light chain expression vectors as previously described ${ }^{4}$. Affinity purification was performed on Äkta Xpress FPLC (Cytiva) operated by Unicorn software version 5.11 (Build 407) using HiTrap Protein A columns (Cytiva) for full length human monoclonal antibodies and CaptureSelect CH1-XL MiniChrom columns (Thermo Fisher Scientific) for Fab fragments, using PBS as mobile phase. Buffer exchange to the appropriate formulation buffer was performed with a HiTrap Fast desalting column (Cytiva). The final products were sterilized by filtration through $0.22-\mu \mathrm{m}$ filters and stored at $4{ }^{\circ} \mathrm{C}$.

Using the Database IMGT (http://www.imgt.org), the $\mathrm{VH}_{\mathrm{H}}$ and $\mathrm{VL}$ germline gene family and the number of somatic mutations were determined by analysing the homology of the $\mathrm{VH}$ and $\mathrm{VL}$ sequences to known human $V, D$ and $/$ genes. Germline-reverted sequences of the $\mathrm{VH}_{\mathrm{H}}$ and $\mathrm{VL}$ were constructed using IMGT/V-QUEST. The S2E12 and S2H97 germline-reverted antibodies (G1m17 allotype) were produced by ATUM. Germline-reverted Fab fragments were generated by digestion of the corresponding IgGs.

Epitope classes shown in Figs. 1a, $2 \mathrm{~g}$ are defined as in Piccoli et al. ${ }^{15}$. In brief, the classification of these epitope classes results from Octet binning experiments using structurally characterized antibodies, structural insights to define the recognition of open-only RBD and ability of antibodies to interfere with RBD binding to ACE2. In particular, site la is accessible only in the open state of RBD and largely overlaps the ACE2 footprint; site Ib is accessible in both open and closed RBD states and overlaps in part the ACE2 footprint; site Ila is in the core RBD (accessible only in the open RBD state) and antibodies binding to this site interfere with binding to ACE2, site IIc is also in the core RBD but targeted by antibodies that do not interfere with binding to ACE2; site IV is fully accessible on both open and closed RBDs and is defined by the footprint of the S309 antibody.

\section{Neutralization of authentic SARS-CoV-2 by entry-inhibition assay}

Neutralization was determined using SARS-CoV-2-Nluc, an infectious clone of SARS-CoV-2 (based on strain 2019-nCoV/USA_WA1/2020) which encodes nanoluciferase in place of the viral ORF7 and demonstrates comparable growth kinetics to wild-type virus ${ }^{44}$. Vero E6 cells (ATCC, CRL-1586) were seeded into black-walled, clear-bottom 96-well plates at $2 \times 10^{4}$ cells per well and cultured overnight at $37^{\circ} \mathrm{C}$. The next day, 9-point fourfold serial dilutions of monoclonal antibodies were prepared in infection media (DMEM $+10 \%$ FBS). SARS-CoV-2-Nluc was diluted in infection media at a final multiplicity of infection (MOI) of 0.01 plaque-forming units (PFU) per cell, added to the monoclonal antibody dilutions and incubated for $30 \mathrm{~min}$ at $37^{\circ} \mathrm{C}$. Medium was removed from the Vero $\mathrm{E} 6$ cells and monoclonal antibody-virus complexes were added and incubated at $37^{\circ} \mathrm{C}$ for $24 \mathrm{~h}$. Media was removed from the cells, Nano-Glo luciferase substrate (Promega) was added according to the manufacturer's recommendations, incubated for $10 \mathrm{~min}$ at room temperature and the luciferase signal was quantified on a Victor Nivo plate reader (Perkin Elmer).

\section{SARS-CoV-2 spike pseudotyped VSV generation and neutralization assay}

Replication-defective VSV pseudoviruses ${ }^{45}$ expressing SARS-CoV-2 spike protein were generated as previously described ${ }^{46}$ with some modifications. Plasmids encoding SARS-CoV-2 spike single-mutant variants were generated by site-directed mutagenesis of the wild-type plasmid, pcDNA3.1(+)-spike-D19 ${ }^{47}$, and plasmids encoding multiply mutated SARS-CoV-2 variants of concern were generated using a multistep overlap extension PCR protocol ${ }^{23,48}$, in which sequential, overlapping fragments were designed to introduce all mutations, which were PCR assembled and cloned into the pcDNA3.1 vector using the Takara In-fusion HD cloning kit following manufacturer's instructions.

Lenti-X 293T (Takara, 632180) cells were seeded in 10-cm dishes at a density of $1 \times 10^{5}$ cells per $\mathrm{cm}^{2}$ and the following day transfected with 
$5 \mu \mathrm{g}$ of spike expression plasmid with TransIT-Lenti (Mirus, 6600) according to the manufacturer's instructions. For the neutralization assays with variants of concern (Figs. 2 d, 3b), Lenti-X 293T cells were seeded in $10-\mathrm{cm}$ dishes at a density of $5 \times 10^{6}$ cells per $\mathrm{cm}^{2}$, and transfected the following day with $10 \mu \mathrm{g}$ of spike expression plasmid. One day after transfection, cells were infected with VSV ( $\mathrm{G}^{*} \Delta \mathrm{G}$-luciferase) (Kerafast, EH1020-p.m.) for $1 \mathrm{~h}$, rinsed three times with PBS, then incubated for an additional $24 \mathrm{~h}$ in complete medium at $37^{\circ} \mathrm{C}$. The cell supernatant was clarified by centrifugation, filtered $(0.45 \mu \mathrm{m})$, aliquoted, and frozen at $-80^{\circ} \mathrm{C}$.

For VSV pseudovirus neutralization assays, Vero E6 cells (ATCC, CRL1586) were grown in DMEM supplemented with $10 \% \mathrm{FBS}$ and seeded into clear bottom white 96 well plates (Costar, 3903) at a density of $2 \times 10^{4}$ cells per well. The next day, monoclonal antibodies were serially diluted in pre-warmed complete media, mixed at a 1:1 ratio with pseudovirus and incubated for $1 \mathrm{~h}$ at $37^{\circ} \mathrm{C}$ in round bottom polypropylene plates. Media from cells was aspirated and $50 \mu \mathrm{l}$ of virus-monoclonal antibody complexes were added to cells and then incubated for $1 \mathrm{~h}$ at $37^{\circ} \mathrm{C}$. An additional $100 \mu \mathrm{l}$ of prewarmed complete media was then added on top of complexes and cells incubated for an additional 16-24 h. Conditions were tested in duplicate wells on each plate and at least six wells per plate contained uninfected, untreated cells (mock) and infected, untreated cells ('no mAb control').

Medium containing virus-monoclonal antibody complexes was then aspirated from cells and $100 \mu \mathrm{l}$ of a 1:4 dilution of Bio-glo (Promega, G7940) in PBS was added to cells. For neutralization assays with variants of concern, $50 \mu \mathrm{l}$ of a 1:2 dilution of SteadyLite Plus (Perkin Elmer) in PBS with $\mathrm{Ca}^{2+}$ and $\mathrm{Mg}^{2+}$ was added to cells in place of Bio-glo. Plates were incubated for $10 \mathrm{~min}$ at room temperature and then were analysed on an Envision plate reader (PerkinElmer), or for variants of concern assays, a Synergy $\mathrm{H} 1 \mathrm{Hyb}$ rid Multi-Mode reader (Biotek).

Relative light unit (RLU) measurements for infected wells were subtracted by the average of RLU values for the mock wells (background subtraction) and then normalized to the average of background subtracted 'no mAb control' RLU values within each plate. Per cent neutralization was calculated by subtracting from 1 the normalized monoclonal antibody infection condition. Data were analysed and visualized with Prism (version 8.4.3). $\mathrm{IC}_{50}$ values were calculated from the interpolated value from the $\log$ (inhibitor) versus response-variable slope (four parameters) nonlinear regression with an upper constraint of $<100$. Neutralization experiments with wild-type SARS-CoV-2S and single-mutant variants were conducted on three independent days, that is, biological replicates, where each biological replicate contains a technical duplicate. $\mathrm{IC}_{50}$ values across biological replicates are presented as geometric mean. The loss or gain of neutralization potency across spike variants was calculated by dividing the variant $\mathrm{IC}_{50}$ by the parental IC $\mathrm{IC}_{50}$ within each biological replicate. Neutralization experiments with SARS-CoV-2 $S$ variants of concern were conducted in biological duplicates, with $\mathrm{IC}_{50}$ values normalized by the corresponding wild-type measurement, and presented as arithmetic mean of the duplicate experiments.

\section{SARS-CoV-2 spike pseudotyped VSV neutralization on 293T-ACE2 cells}

To investigate the effect of ACE2 expression on S2H97 neutralization, Vero E6 cells were seeded at 20,000 cells per well in black clear-bottom 96-well plates. 293T-ACE2 cells ${ }^{31}$ were seeded at 35,000 cells per well in black clear-bottom 96-well plates that had been pre-coated with poly-D-lysine (Gibco). The next day, SARS-CoV-2 spike-pseudotyped VSV neutralizations with S2E12, S309 and S2H97 were performed as described above. Neutralizations were performed in triplicate wells.

Sarbecovirus spike pseudotyped VSV neutralization by S2H97

Mammalian expression constructs (pcDNA3.1(+) or pTwist-CMV) encoding the spike proteins from various sarbecoviruses with a $\mathrm{C}$-terminal deletion of 19 amino acids (D19) were synthesized for SARS-CoV-2 (GenBank: QOU99296.1), SARS-CoV-1Urbani (GenBank: AAP13441.1), hCoV-19/pangolin/Guangdong/1/2019 (GD-pangolin-CoV, GenBank: QLR06867.1), pangolin coronavirus Guanxi-2017 (GX-pangolin-CoV, GenBank: QIA48623.1), and bat sarbecovirus WIV1 (WIV1, GenBank: AGZ48828.1). Lenti-X 293T cells (Takara, 632180) were seeded in $15-\mathrm{cm}$ dishes such that the cells would reach $80 \%$ confluency after culturing overnight. The following day, cells were transfected using TransIT-Lenti (Mirus, 6600) according to the manufacturer's instructions. One day after transfection, cells were infected with VSV (G* $\Delta \mathrm{G}$-luciferase) (Kerafast, EH1020-p.m.). The supernatant containing sarbecovirus pseudotyped VSV was collected 2 days after transfection, centrifuged at $1,000 \mathrm{~g}$ for $5 \mathrm{~min}$, aliquoted and frozen at $-80^{\circ} \mathrm{C}$.

For neutralization assays, cells supporting robust pseudovirus infection were seeded into clear bottom white-walled 96-well plates at 20,000 cells per well in $100 \mu \mathrm{l}$ culture media. Vero E6 cells were used for VSV-SARS-CoV-2, VSV-SARS-CoV-1, and VSV-GD-pangolin-CoV. BHK-21 cells (ATCC, CCL-10) stably expressing ACE2 were used for VSV-GX-pangolin-CoV and VSV-WIV1. After culturing cells overnight, 1:3 serial dilutions of antibody were prepared in DMEM in triplicate. Pseudovirus was diluted in DMEM and added to each antibody dilution such that the final dilution of pseudovirus was 1:20. Pseudovirus-antibody mixtures were incubated for $1 \mathrm{~h}$ at $37^{\circ} \mathrm{C}$. Medium was removed from the cells and $50 \mu \mathrm{l}$ of pseudovirus:antibody mixtures were added. One hour after infection, $50 \mu \mathrm{l}$ of culture medium was added to wells containing pseudovirus:antibody mixtures and incubated overnight at $37^{\circ} \mathrm{C}$. Medium was then removed and $100 \mu \mathrm{l}$ of 1:1 diluted DPBS:Bio-Glo (Promega, G7940) luciferase substrate was added to each well. The plate was shaken at $300 \mathrm{rpm}$ at room temperature for 10 min after which RLU reading were taken on an EnSight (Perkin Elmer) microplate reader. Per cent neutralization was determined by first subtracting the mean background (cells with luciferase substrate alone) RLU values of 6 wells per plate for all data points. Per cent neutralization for each antibody concentration was calculated relative to no-antibody control wells for each plate. Per cent neutralization data were analysed and graphed using Prism (GraphPad, v9.0.1). Absolute $\mathrm{IC}_{50}$ values were calculated by fitting a curve using a nonlinear regression model (variable slope, 4 parameters) and values were interpolated from the curve at $y=50 \%$. The geometric mean from at least two independent experiments was calculated using Excel (Microsoft, version 16.45).

\section{Sarbecovirus spike pseudotyped VSV neutralization by S2E12} Spikes from SARS-CoV-2 (CAD0240757.1), RaTG13 (QHR63300.2), GD-pangolin (QLR06867.1), GX-pangolin (QIA48623.1), SARS-CoV-1 Tor2 (YP009825051), WIV1 (AGZ48831.1) and WIV16 (ALK02457.1) were used to pseudotype VSV. To produce pseudotyped viruses, HEK293T/17 (ATCC, CRL-11268) seeded in 10-cm dishes in DMEM supplemented with $10 \%$ FBS, $1 \%$ penicillin-streptomycin were transfected with plasmids using lipofectamine 2000 (Life Technologies) following manufacturer's instructions. One day post-transfection, cells were infected with VSV ( $\mathrm{G}^{*} \Delta \mathrm{G}$-luciferase) for $2 \mathrm{~h}$ and washed 4 times with DMEM, before adding medium supplemented with anti-VSV-G antibody (I1-mouse hybridoma supernatant at 1:50 dilution, from CRL-2700, ATCC). Pseudotyped particles were collected $18 \mathrm{~h}$ after inoculation, clarified by centrifugation at $2,000 \mathrm{~g}$ for $5 \mathrm{~min}$, concentrated $10 \times$ with a $30 \mathrm{kDa}$ cut-off membrane filter, and stored at $-80^{\circ} \mathrm{C}$. For S2E12 neutralization experiments, $293 \mathrm{~T}$ cells stably expressing ACE2 (BEI \#NR-52511) ${ }^{43}$ in DMEM supplemented with $10 \% \mathrm{FBS}$ and $1 \%$ penicillin-streptomycin were seeded at 40,000 cells per well in clear-bottom white-walled 96 -well plates and cultured overnight at $37^{\circ} \mathrm{C}$. Twelve threefold serial dilutions of S2E12 antibody were prepared in DMEM, and antibody dilutions were mixed 1:1 with pseudotyped VSV in the presence of 1:50 diluted anti-VSV-G antibody. After 45 min incubation at $37^{\circ} \mathrm{C}, 40 \mu$ lof antibody-virus mixture was added to cells, and $40 \mu \mathrm{l}$ DMEM was added $2 \mathrm{~h}$ post-infection. After 17-20 h, $50 \mu \mathrm{l}$ One-Glo-EX substrate (Promega) was added to the cells. 
Cells were incubated in the dark for 5-10 min before luminescence reading on a Varioskan LUX plate reader (Thermo Fisher Scientific). Relative luciferase unit values were converted to percentage of neutralization and plotted with a nonlinear regression curve fit in GraphPad Prism. Measurements were performed in duplicate with two independent productions of pseudotyped virus.

\section{Recombinant protein production}

SARS-CoV-2 RBD wild-type protein for SPR binding assays (with $\mathrm{N}$-terminal signal peptide and $\mathrm{C}$-terminal thrombin cleavage site-TwinStrep-8 $\times$ His-tag) was expressed in Expi293F (Thermo Fisher Scientific) cells at $37^{\circ} \mathrm{C}$ and $8 \% \mathrm{CO}_{2}$. Transfections were performed using the ExpiFectamine 293 Transfection Kit (Thermo Fisher Scientific). Cell culture supernatants were collected three days after transfection and supplemented with $10 \times \mathrm{PBS}$ to a final concentration of $2.5 \times \mathrm{PBS}$ (342.5 mM NaCl, 6.75 mM KCland $29.75 \mathrm{mM}$ phosphates). SARS-CoV-2 RBDs were purified using 1 or $5 \mathrm{ml}$ HisTALON Superflow cartridges (Takara Bio) and subsequently buffer exchanged into $1 \times$ HBS-N buffer (Cytiva) or PBS using a Zeba Spin Desalting (Thermo Fisher Scientific) or HiPrep 26/10 (Cytiva) desalting column.

SARS-CoV-2 RBD wild-type for crystallization (with $\mathrm{N}$-terminal signal peptide, ETGT and C-terminal $8 \times$ His-tag) was expressed similarly as described above in the presence of $10 \mu \mathrm{M}$ kifunensine. Cell culture supernatant was collected four days after transfection and supplemented with $10 \times$ PBS to a final concentration of $2.5 \times$ PBS. Protein was purified using a $5 \mathrm{ml}$ HisTALON Superflow cartridge followed by size-exclusion chromatography on a Superdex 200 Increase $10 / 300$ GL column (Cytiva) equilibrated in $20 \mathrm{mM}$ Tris- $\mathrm{HCl} \mathrm{pH} \mathrm{7.5,150} \mathrm{mM} \mathrm{NaCl}$. For crystallization of the RBD-S2X259-S2H97 and RBD-S2E12-S304-S309 Fab complexes, RBD was deglycosylated by overnight incubation with EndoH glycosidase at $4{ }^{\circ} \mathrm{C}$.

RBDs from other sarbecoviruses for SPR (with $\mathrm{N}$-terminal signal peptide and C-terminal thrombin cleavage site-TwinStrep- $8 \times$ His-tag) were expressed in Expi293F cells at $37^{\circ} \mathrm{C}$ and $8 \% \mathrm{CO}_{2}$. Cells were transfected using PEI MAX (Polysciences) at a DNA:PEI ratio of 1:3.75. Transfected cells were supplemented three days after transfection with $3 \mathrm{gl}^{-1}$ glucose (Bioconcept) and $5 \mathrm{~g} \mathrm{l}^{-1}$ soy hydrolysate (Sigma-Aldrich). Cell culture supernatant $(423 \mathrm{ml})$ was collected 7 days after transfection and supplemented with $47 \mathrm{ml} 10 \times$ binding buffer $(1 \mathrm{M}$ Tris- $\mathrm{HCl}, 1.5 \mathrm{M}$ $\mathrm{NaCl}, 20 \mathrm{mM}$ EDTA, pH 8.0) and $25 \mathrm{ml}$ BioLock (IBA) and incubated on ice for $30 \mathrm{~min}$. Proteins were purified using a $5-\mathrm{ml}$ Strep-Tactin XT Superflow high-capacity cartridge (IBA) followed by buffer exchange to PBS using HiPrep 26/10 desalting columns (Cytiva).

Prefusion-stabilized SARS-CoV-2 spike proteins for SPR (residues 14-1211, either D614 or D614G), containing the 2P and Furin cleavage site mutations ${ }^{49}$ with a mu-phosphatase signal peptide and a C-terminal Avi-8 $\times$ His-C-tag or C-terminal $8 x$ His-Avi-C-tag were expressed in Freestyle 293-F cells (Thermo Fisher Scientific, R79007) at $37^{\circ} \mathrm{C}$ and $8 \% \mathrm{CO}_{2}$. Transfections were performed using $293 \mathrm{fectin}$ as a transfection reagent. Cell culture supernatant was collected after three days and purified over a $5 \mathrm{ml} \mathrm{C}$-tag affinity matrix. Elution fractions were concentrated and injected on a Superose 6 Increase $10 / 300$ GL column (Cytiva) with $50 \mathrm{mM}$ Tris- $\mathrm{HCl} \mathrm{pH} 8.0$ and $200 \mathrm{mM} \mathrm{NaCl}$ as running buffer.

SARS-CoV-2 HexaPro spike protein for cryoEM analysis was produced in Freestyle 293-F cells grown in suspension using FreeStyle 293 expression medium (Life Technologies) at $37^{\circ} \mathrm{C}$ in a humidified $8 \% \mathrm{CO}_{2}$ incubator rotating at $130 \mathrm{rpm}$. The cultures were transfected using PEI $\left(9 \mu \mathrm{g} \mathrm{ml}^{-1}\right)$ with cells grown to a density of 2.5 million cells per ml and cultivated for three days. The supernatants were collected and cells resuspended for another three days, yielding two collections from each such culture. Spike proteins were purified from clarified supernatants using a $5 \mathrm{ml}$ cobalt affinity column (Cytiva, HiTrap TALON crude), concentrated and flash frozen in a buffer containing $20 \mathrm{mM}$ Tris pH 8.0 and $150 \mathrm{mM} \mathrm{NaCl}$ before analysis.

SARS-CoV-2 S native-like ectodomain trimer for refolding assays was engineered with a mu-phosphatase signal peptide beginning at $14 \mathrm{Q}$, a mutated $\mathrm{S} 1 / \mathrm{S} 2$ cleavage site (SGAR), and a TEV cleavage, fold-on trimerization motif, and $8 x$ His tag appended to the $C$ terminus (K1211). Native-like spike was expressed and purified as described for SARS-CoV-2 HexaPro spike above.

Recombinant hACE2 for SPR (residues 19-615 from Uniprot Q9BYF1 with a C-terminal AviTag-10 $\times$ His-GGG-tag, and N-terminal signal peptide) was produced by ATUM. Protein was purified via Ni Sepharose resin followed by isolation of the monomeric hACE2 by size exclusion chromatography using a Superdex 200 Increase 10/300 GL column (Cytiva) pre-equilibrated with PBS.

\section{SPR binding assays}

SPR binding measurements were performed using a Biacore T200 instrument with CM5 sensor chip covalently immobilized with StrepTactin XT to capture recombinant RBD proteins (data in Fig. 1a, Extended Data Figs. 1c, 4f, i, I). Running buffer was Cytiva HBS-EP+ (pH 7.4). All measurements were performed at $25^{\circ} \mathrm{C}$. Fab (or hACE2) analyte concentrations were $11,33,100$ and $300 \mathrm{nM}$, run as single-cycle kinetics. Double reference-subtracted data were fit to a 1:1 binding model using Biacore T200 Evaluation (version 3.1) or Biacore Insight Evaluation (version 2.0.15) software. $K_{\mathrm{D}}$ values above $1 \mu \mathrm{M}$ were determined from fits where the maximum SPR signal at saturation $\left(R_{\max }\right)$ was set as a constant determined from results for higher affinity analytes binding to the same RBD at the same surface density. Data where averages are not given are representative of duplicate or triplicate measurements (except measurements with germline Fabs, which were singleton measurements).

To corroborate the SARS-CoV-2 RBD binding measurements, experiments were also performed in two additional formats, both with monovalent analytes (data in Extended Data Table 1): (1) Fab binding to SARS-CoV-2 spike ectodomain was measured using CM5 sensor chips immobilized with anti-AviTag pAb (Genscript, A00674-40) for capturing $S$, other experiment parameters same as above, and (2) RBD binding to IgG was measured using CM5 sensor chips immobilized with anti-human Fc pAb (Southern Biotech, 2014-01) for capturing IgG, with RBD analyte concentrations of $3.1,12.5$, and $50 \mathrm{nM}$, other experiment parameters same as above. Fit results yield an apparent $K_{\mathrm{D}}$ for the spike-binding experiments because the kinetics also reflect spike conformational dynamics. Spike ectodomain was D614G with C-terminal 8xHis-Avi-C-tag for all measurements except S2X58 binding was performed with D614 spike with C-terminal Avi- $8 \times$ His-C-tag. For the comparison of mature and germline-reverted antibody binding to RaTG13, the data reported are from experiment format (2) with IgG as ligand. These data (and others indicated in Extended Data Table 1 as 'biphasic kinetics') were fit to a heterogeneous ligand model, due to an artefactual kinetic phase with very slow dissociation that often arises when RBD is an analyte; the lower affinity of the two $K_{\mathrm{D}}$ values reported by the fit is given as the $K_{\mathrm{D}}$ (the two $K_{\mathrm{D}}$ values are separated by at least one order of magnitude).

\section{Deep mutational scanning mutant escape profiling}

We used a previously described deep mutational scanning approach ${ }^{3}$ to comprehensively identify RBD mutations that escape binding by each antibody. This approach leverages duplicate RBD mutant libraries ${ }^{26}$, which contain virtually all of the 3,819 possible amino acid mutations in the background of the Wuhan-Hu-1 RBD sequence. Library variants were previously linked to short identifier barcode sequences and sorted to purge the library of variants that strongly decrease ACE2 binding affinity or expression of folded $\mathrm{RBD}^{3}$.

We first used an isogenic yeast strain expressing the unmutated SARS-CoV-2 RBD and flow cytometry to identify the EC 90 of each antibody's binding to yeast-displayed SARS-CoV-2RBD. We then performed library selections as previously described ${ }^{3,20}$, labelling libraries with the EC90 concentration of antibody to standardize escape mutation sensitivity across selections. In brief, libraries of yeast were induced 
for surface expression, washed, and labelled with the primary antibody for $1 \mathrm{~h}$ at room temperature. Cells were washed, and secondarily labelled with 1:200 PE-conjugated goat anti-human-IgG antibody (Jackson ImmunoResearch 109-115-098) to label for bound antibody, and 1:100 FITC-conjugated chicken anti-Myc-tag (Immunology Consultants Lab, CYMC-45F) to label for RBD surface expression. We prepared controls for setting FACS selection gates by labelling yeast expressing the unmutated SARS-CoV-2 RBD with the same antibody concentration as library selections $(1 \times), 100 \times$ reduced antibody concentration to illustrate the effect of mutations with $100 \times$-reduced affinity, and $0 \mathrm{ng} \mathrm{ml}^{-1}$ antibody to illustrate complete loss of antibody binding. Representative selection gates are shown in Extended Data Fig. $2 \mathrm{~b}$. Gates were set and sorting performed with FACSDiva software (version 6.1.3). We sorted approximately $7.5 \times 10^{6} \mathrm{RBD}^{+}$cells per library on a BD FACSAria II, collecting yeast cells from the antibody-escape sort bin (fractions of library falling into antibody escape bin given in Extended Data Fig. 2c). Sorted cells were recovered overnight, plasmids were extracted from the pre-sort and antibody-escape populations, and variant-identifier barcode sequences were PCR amplified and sequenced on an Illumina HiSeq $2500^{3,26}$.

As previously described ${ }^{3,20}$, sequencing counts pre-and post-selection were used to estimate the 'escape fraction' for each library variant, which reflects the fraction of yeast expressing a variant that fall into the antibody-escape FACS bin. In brief, we used the dms_variants package (https://jbloomlab.github.io/dms_variants/, version 0.8.2) to process Illumina sequences into variant counts pre- and post-selection using the barcode/RBD variant lookup table from Starr et al. ${ }^{26}$. We then computed per-variant escape fraction of variant $v\left(E_{v}\right)$ as: $E_{v}=F \times\left(n_{v_{\text {post }}} / N_{\text {post }}\right) /\left(n_{v_{\text {pre }}} / N_{\text {pre }}\right)$, where $F$ is the total fraction of the library that escapes antibody binding (Extended Data Fig. $2 \mathrm{c}$ ), $n_{v_{\text {post }}}$ and $n_{v_{\text {pre }}}$ are the sequencing counts of variant $v$ in the RBD library after and before FACS selection, respectively (with a pseudocount of 0.5 added to all counts), and $N_{\text {post }}$ and $N_{\text {pre }}$ are the total counts of all variants after and before FACS selection, respectively. We then applied computational filters to remove variants with low pre-sort sequencing counts or highly deleterious mutations that might cause artefactual antibody escape due to global unfolding or loss of expression of RBD on the cell surface. Specifically, we filtered out variants whose pre-selection sequencing counts were lower than the 99th percentile counts of variants containing premature stop codons, which were largely purged by the prior sorts for RBD expressing and ACE2-binding RBD variants. We also removed variants with ACE2 binding scores below -2.35 or RBD expression scores below -1 , and variants containing individual mutations with effects below these thresholds, using the variant- and mutationlevel deep mutational scanning measurements of Starr et al. ${ }^{26}$. We also filtered out rare mutations with low coverage in the libraries, retaining mutations that were sampled on at least one single-mutant barcoded variant or at least two multiply mutated variants in each replicate. Last, to decompose single-mutation escape fractions for each antibody, we implemented global epistasis model ${ }^{50}$ using the dms_variants package to estimate the effect of each individual amino acid mutation, exactly as described in ref. ${ }^{20}$.

Antibody escape selections were conducted in full duplicate using independently generated and assayed SARS-CoV-2 mutant libraries (see correlations in Extended Data Fig. 2e,f). The reported escape fractions throughout the paper are the average across the two replicates, and these final per-mutation escape fractions are provided on GitHub (https://github.com/jbloomlab/SARS-CoV-2-RBD_MAP_Vir_mAbs/blob/ main/results/supp_data/vir_antibodies_raw_data.csv). Interactive visualizations of antibody escape maps (https://jbloomlab.github. io/SARS-CoV-2-RBD_MAP_Vir_mAbs) were created using dms-view ${ }^{51}$.

\section{Sarbecovirus library binding assays}

A curated set of all unique sarbecovirus RBD amino acid sequences was gathered, including the sarbecovirus RBD sequence set reported by Letko et al. ${ }^{7}$, along with additional unique RBD sequences among SARS-CoV-1 epidemic strains reported by Song et al. ${ }^{52}$, BtKY72 $2^{53}$ and new sarbecovirus sequences RmYNO2 $2^{54}$, GD-pangolin-CoV (consensus RBD reported in figure 3a of Lam et al. $\left.{ }^{55}\right)$, and GX-pangolin- $\mathrm{CoV}^{55}(\mathrm{P} 2 \mathrm{~V}$, ambiguous nucleotide within codon 515 (SARS-CoV-2 spike numbering) resolved to retain $\mathrm{F} 515$, which is conserved in all other sarbecoviruses). A list of all RBDs and sequence accession numbers is available on GitHub (https://github.com/jbloomlab/SARSr-CoV_RBD_MAP/blob/main/data/ RBD_accessions.csv).

To define clades of sarbecovirus RBDs, an alignment of amino acid RBD sequences was generated using mafft ${ }^{56}$ with gap opening penalty 4.5 (alignment available on GitHub: https://github.com/jbloomlab/ SARSr-CoV_RBD_MAP/blob/main/data/RBD_aa_aligned.fasta). The corresponding nucleotide sequence alignment was generated from the amino acid alignment using PAL2NAL ${ }^{57}$. The gene sequence phylogeny was inferred using RAxML version 8.2.12 $2^{58}$, with the GTRGAMMA substitution model and a partition model with separate parameters for first, second and third codon positions. The Hibecovirus RBD sequence Hp-Zhejiang2013 (GenBank: KF636752) was used as an outgroup for rooting of the sarbecovirus phylogeny.

All unique sarbecovirus RBD protein-coding sequences were ordered from IDT, Twist or Genscript, and cloned into our yeast display vector $^{26}$. Sequences were pooled and appended with downstream 16-nt barcode sequences according to the protocol described in Starr et al. ${ }^{26}$. Long-read circular consensus sequences spanning the 16-nt barcode and RBD genotype were gathered on a PacBio Sequel v2.0 and processed exactly as described in Starr et al. ${ }^{26}$. This yielded a barcode:variant lookup table for the sarbecovirus RBD library analogous to that used for SARS-CoV-2 mutant libraries. This table is available on GitHub (https:// github.com/jbloomlab/SARSr-CoV_RBD_MAP/blob/main/data/barcode _ variant_table.csv).

The pooled sarbecovirus RBD library was labelled, sorted, and quantified as described for the SARS-CoV-2 mutant libraries above, except we only sorted around 1 million $\mathrm{RBD}^{+}$cells owing to the reduced library size. Sequencing and quantification of per-variant antibody escape was conducted as described above. Data for the HKU3-8 RBD are not shown, as this RBD was not successfully expressed in our yeast-display platform. For several antibodies, we performed a secondary experiment, selecting the sarbecovirus RBD library with a more stringent 'full escape' gate to select out only variants exhibiting complete loss of binding (Extended Data Fig. 2b, c).

For follow-up quantitative binding assays, select sarbecovirus RBDs were cloned into the yeast-display platform as isogenic stocks. Binding assays were conducted across a titration series of antibody in 96-well plates, and binding at each antibody concentration (geometric mean fluorescence intensity in the PE channel among $\mathrm{RBD}^{+}\left(\mathrm{FITC}^{+}\right)$cells) was determined via flow cytometry and fit to a four-parameter Hill curve to identify the $\mathrm{EC}_{50}$ (midpoint).

\section{Analysis of mutations in natural SARS-CoV-2 sequences}

All spike sequences on GISAID ${ }^{59}$ as of 2 May 2021, were downloaded and aligned using maff $\mathrm{t}^{56}$. Sequences from non-human origins, sequences with gaps or ambiguous characters in the RBD, and sequences with more than eight amino acid differences from the Wuhan-Hu-1 reference sequence (GenBank MN908947, residues N331-T531) were removed. We determined mutation frequencies compared with Wuhan-Hu-1 reference from this final alignment of $1,190,241$ sequences. We acknowledge all contributors to the GISAID EpiCoV database for their sharing of sequence data. The contributors to GISAID EpiCoV are listed at https:// github.com/jbloomlab/SARS-CoV-2-RBD_MAP_Vir_mAbs/blob/main/ data/gisaid_hcov-19_acknowledgement_table_2021_03_04.pdf.

\section{Quantitative summary metrics of antibody properties}

The relative epitope size of an antibody was calculated as the sum of per-mutant escape fractions that are at least five times the global 
median escape fraction (to minimize the impact of variation in background noise on the summation). For this summation, escape fractions were normalized to the maximum per-mutation escape fraction, to account for slight variation in the largest per-mutation escape fraction measured between selections.

The relative escapability of an antibody was calculated the same as relative epitope size, but each mutation was multiplied by two weighting factors scaled from 0 to 1 that reflect the impact of that mutation on ACE2-binding affinity and RBD expression as measured in our prior deep mutational scan ${ }^{26}$. The relationship between weighting factors and mutation effect on each property is shown in Extended Data Fig. 3a. Mutations with $<-1$ effect on either property are effectively zeroed out in the escapability summation. Mutations with effects between -1 and 0 have intermediate weights, and mutations with 0 or positive effects are assigned weight factors of 1 .

Antibody susceptibility to escape by natural SARS-CoV-2 mutations was calculated as the summed GISAID frequencies of all escape mutations, where escape mutations (all labels in Extended Data Fig. $3 \mathrm{c}$ ) are defined as those with escape fraction greater than five times the median escape fraction as above. These summed natural escape frequencies are tabulated in the plot headers in Extended Data Fig. 3c.

The summary breadth of an antibody was calculated from the sarbecovirus RBD library escape selection using the standard gating (Extended Data Fig. 4b), only. Although we have various follow-up binding data illustrating reduced affinity binding for some 'escaped' sarbecovirus RBDs, these follow-up experiments were not conducted systematically for all antibody-RBD combinations, and therefore would bias breadth estimates. Breadth of binding was calculated as the frequency of all sarbecovirus RBDs that are bound with affinity within the FACS selection gating threshold, weighted by clade representation. Breadth was normalized to give equal representation to each of the four sarbecovirus clades to account for different depth of sampling. Within the SARS-CoV-1 clade, all human 02/03 strains and civet and human $03 / 04$ strains were similarly down-weighted to each represent $1 / 8$ of the possible breadth within the SARS-CoV-1 clade (together with the six bat sarbecoviruses in this clade). As an example, breadth for $\mathrm{S} 304$ is calculated as $[4 / 4+([6 / 6]+[6 / 6]+5) / 8+2 / 2+0 / 21] / 4=0.72$, based on the data shown in Extended Data Fig. $4 \mathrm{~b}$.

\section{Multidimensional scaling projection of antibody epitopes}

Multidimensional scaling projection in Fig. 4 was performed using the Python scikit-learn package. We first computed the similarity and dissimilarity in the sites of escape between each pair of antibodies, exactly as described in Greaney et al. ${ }^{3}$, and performed metric multidimensional scaling with two components on the matrix of dissimilarities between all antibody pairs. Antibodies in this layout were coloured with pie charts proportional to the total squared site-wise escape that falls into the labelled structural regions (RBM, residues 437 to 508, ACE2 contact defined as 4 Åcut-off based on 6MOJ crystal structure ${ }^{60}$, and core RBD otherwise). In this layout, we included all of our previously published antibodies for which we have performed escape mapping via this same approach. These antibodies and their citations include: S2X25937; LY-CoV555 ${ }^{21}$; COV2-2196 and COV2-2130 ${ }^{36}$; REGN10933, REGN10987 and LY-CoV016 ${ }^{20}$; and all other COV2 antibodies and CR3022 ${ }^{3}$.

For Fig. 4b-d and Extended Data Fig. 7c, we coloured the antibodies within this layout according to various antibody properties. When appropriate, we also coloured these previously assayed antibodies, as described below. Extended Data Fig. 7d and the scatter plots in Fig. 4e-g show the relationships between properties for antibodies specifically in this study (and S2X259) for the most direct comparability.

Antibody neutralization potencies illustrated in Fig. $4 \mathrm{~b}$ incorporate the authentic SARS-CoV-2 neutralization IC $_{50}$ values as reported in this study (Fig. 1a), together with the live SARS-CoV-2 neutralization $\mathrm{IC}_{50}$ values for the COV 2 antibodies reported by Zost et al. ${ }^{10}$. We acknowledge that it is imperfect to compare neutralization potencies reported from different labs on different antibody batches, though in this case, both sets are indeed neutralization potencies with authentic virus. We therefore do not directly compare these two sets of measurements in a quantitative manner, but we do note that their joint inclusion in Fig. 4b supports the dichotomy between neutralization potency of core RBD versus RBM antibodies which is supported by either neutralization panel alone.

Sarbecovirus breadth illustrated in Fig. 4c incorporates the pan-sarbecovirus breadth measurements reported in the current study together with more limited breadth measurements for antibodies reported in previous publications. These previously published experiments determined binding within a more restricted sarbecovirus RBD set present in our libraries (SARS-CoV-2, RaTG13, GD-pangolin, SARS-CoV-1 (Urbani), LYRa11 and WIV1). We calculated breadth from this incomplete sarbecovirus sequence set for comparison, but note that these antibodies are limited to a relative breadth of 0.5 because no RBDs from the Africa/Europe or non-ACE2-using Asia clades were included. However, as with neutralization, inclusion of these antibodies nonetheless emphasizes the core RBD-RBM dichotomy in sarbecovirus breadth established by our primary panel.

For illustrations of epitope size and escapability in Fig. 4d and Extended Data Fig. 7c, we calculated these quantities for our previously profiled antibodies as described above. We excluded the antibodies profiled in Greaney et al. ${ }^{3}$, as these assays were performed on a prior version of our SARS-CoV-2 mutant library that exhibited different quantitative features of absolute escape, complicating its quantitative comparison to extent of escape for antibodies profiled in this and our other studies, which all use the same library.

Structural mappings around the perimeter of Fig. 4 a were created by mapping total site-wise escape to the B-factor column of PDB structures. Footprints were defined as residues within a $5 \AA$ cut-off of antibody heavy atoms. Structures used were those described in this paper, or previously published structures: ACE2-bound RBD (6MOJ) $)^{60}$, CR3022-bound RBD (6W41) ${ }^{61}$, REGN10987-and REGN10933-bound RBD $(6 X D G)^{62}$, CB6- (LY-CoV016) bound RBD $(7 C 01)^{63}$, and S304, S309 and S2H14-bound RBD (7JX3) $)^{15}$.

\section{RBDELISA}

Ninety-six half-area well-plates (Corning, 3690) were coated over-night at $4{ }^{\circ} \mathrm{C}$ with $25 \mu \mathrm{l}$ of sarbecoviruses RBD proteins at $5 \mu \mathrm{g} \mathrm{ml}^{-1}$ in PBS $\mathrm{pH}$ 7.2. Plates were blocked with PBS 1\% BSA (Sigma-Aldrich, A3059) and subsequently incubated with serial dilutions of monoclonal antibody for $1 \mathrm{~h}$ at room temperature. After 4 washing steps with PBS 0.05\% Tween 20 (PBS-T) (Sigma-Aldrich, 93773), goat anti-human IgG secondary antibody (Southern Biotech, 2040-04) was added and incubated for $1 \mathrm{~h}$ at room temperature. Plates were then washed 4 times with PBS-T and 4-nitrophenyl phosphate (pNPP, Sigma-Aldrich, 71768) substrate was added. After 30 min of incubation, absorbance at $405 \mathrm{~nm}$ was measured by a plate reader (Biotek) and data were plotted using GraphPad Prism.

\section{Binding to cell surface-expressed sarbecovirus $S$ proteins by flow cytometry}

ExpiCHO-S cells were seeded at $6 \times 10^{6}$ cells per $\mathrm{ml}$ in a volume of $5 \mathrm{ml}$ in a 50-ml bioreactor. Spike coding plasmids were diluted in cold OptiPROSFM, mixed with ExpiFectamine CHO Reagent (Life Technologies) and added to the cells. Transfected cells were then incubated at $37^{\circ} \mathrm{C}$ with $8 \% \mathrm{CO}_{2}$ with an orbital shaking speed of $120 \mathrm{rpm}$ (orbital diameter of $25 \mathrm{~mm}$ ) for $42 \mathrm{~h}$. Transiently transfected ExpiCHO-S cells were collected and washed two times in wash buffer (PBS, 1\% BSA, $2 \mathrm{mM}$ EDTA). Cells were counted and distributed into round bottom 96-well plates (Corning) and incubated with $10 \mathrm{\mu g} \mathrm{ml}^{-1}$ S2H97, S2X35 or S309 monoclonal antibody. Alexa Fluor647-labelled Goat Anti-Human IgG secondary Ab (Jackson ImmunoResearch 109-607-003) was prepared at $1.5 \mu \mathrm{g} \mathrm{ml}^{-1}$ and added to cells after two washing steps. Cells were then 
washed twice and resuspended in wash buffer for data acquisition on a ZE5 cytometer (Bio-Rad).

\section{Crystallization, data collection, structure determination and analysis}

To form RBD-Fab complexes for crystallization, SARS-CoV-2 RBD was mixed with a 1.3-fold molar excess of each Fab and incubated on ice for 20-60 min. Complexes were purified on a Superdex 200 Increase $10 / 300 \mathrm{GL}$ column (Cytiva) preequilibrated with $20 \mathrm{mM} \mathrm{Tris-HCl} \mathrm{pH} 7.5$ and $150 \mathrm{mM} \mathrm{NaCl}$. Crystals of the RBD-Fab complexes were obtained at $20^{\circ} \mathrm{C}$ by sitting-drop vapour diffusion.

For the SARS-CoV-2 RBD-S2X35-S309 complex, a total of $200 \mathrm{nl}$ complex at $5.4 \mathrm{mg} \mathrm{ml}^{-1}$ was mixed with $100 \mathrm{nl}$ mother liquor solution containing $1.85 \mathrm{M}$ ammonium sulfate, $0.1 \mathrm{M}$ Tris $\mathrm{pH} 8.17,0.8 \%(\mathrm{w} / \mathrm{v})$ polyvinyl alcohol, $1 \%(\mathrm{v} / \mathrm{v}) 1$-propanol, and $0.01 \mathrm{M} \mathrm{HEPES} \mathrm{pH} \mathrm{7.} \mathrm{Crystals} \mathrm{were}$ flash frozen in liquid nitrogen using the mother liquor solution supplemented with $20 \%$ glycerol for cryoprotection. Data were collected at Beamline 9-2 of the Stanford Synchrotron Radiation Lightsource facility in Stanford, CA and processed with the XDS software package (version 31 January 2020$)^{64}$ to $1.83 \AA ̊$ in space group C222. The RBD-S2X35-S309 Fab complex structure was solved by molecular replacement using phaser ${ }^{65}$ from a starting model consisting of SARS-CoV-2 RBD-S309 Fab (PDB 7JX3) and a homology model for the S2X35 Fab built using the Molecular Operating Environment (MOE) software package from the Chemical Computing Group (https://www.chemcomp.com).

For the SARS-CoV-2 RBD-S2H97-S2X259 Fab complex, $200 \mathrm{nl}$ complex at $5.7 \mathrm{mg} \mathrm{ml}^{-1}$ was mixed with $200 \mathrm{nl}$ mother liquor solution containing $0.12 \mathrm{M}$ monosaccharides mix, 20\% (v/v) ethylene glycol, 10\% (w/v) PEG $8000,0.1 \mathrm{M}$ Tris (base)/bicine $\mathrm{pH} 8.5,0.02 \mathrm{M}$ sodium chloride, $0.01 \mathrm{M}$ MES pH 6 and 3\% (v/v) Jeffamine ED-2003. Crystals were flash frozen in liquid nitrogen. Data were collected at Beamline 9-2 of the Stanford Synchrotron Radiation Lightsource facility in Stanford, CA. Data were processed with the XDS software package (version Jan 31,2020$)^{64}$ for a final dataset of $2.65 \AA$ in space group $P 2_{1}$. The RBD-S2H97-S2X259 Fab complex structure was solved by molecular replacement using phaser from a starting model consisting of SARS-CoV-2 RBD (PDB 7JX3) and homology models for the S2H 97 and S2X259 Fabs built using the MOE software package.

For the SARS-CoV-2 RBD-S2E12-S304-S309 Fab complex, $200 \mathrm{nl}$ complex at $4.5 \mathrm{mg} \mathrm{ml}^{-1}$ was mixed with $100 \mathrm{nl}$ of $0.09 \mathrm{M}$ phosphate/ citrate $\mathrm{pH} 5.5,27 \%(\mathrm{v} / \mathrm{v}) \mathrm{PEG}$ smear low, $4 \%(\mathrm{v} / \mathrm{v})$ polypropylene glycol 400 and $0.02 \mathrm{M}$ imidazole $\mathrm{pH} 7$ or $100 \mathrm{nl}$ of $0.09 \mathrm{M}$ phosphate/citrate $\mathrm{pH} 5.5,27 \%(\mathrm{v} / \mathrm{v})$ PEG smear low, $0.01 \mathrm{M}$ potassium/sodium phosphate $\mathrm{pH} 7,1 \%$ (v/v) PPGBA 230 and 1.5\% (v/v) PPGBA 400. Crystals were flash frozen in liquid nitrogen. Data were collected at the Molecular Biology Consortium beamline 4.2.2 at the Advanced Light Source synchrotron facility in Berkeley, CA. Datasets from two crystals from the two conditions were individually processed and then merged with the XDS software package (version 31 January 2020) ${ }^{64}$ for a final dataset of $2.93 \AA ̊$ in spacegroup $/ 4_{1} 22$. The RBD-S2E12-S304-S309Fab complexstructure was solved by molecular replacement using phaser from starting models consisting of SARS-CoV-2 RBD-S304-S309 Fab (PDB 7JX3) and S2E12 (PDB 7K3Q).

For all structures, several subsequent rounds of model building and refinement were performed using Coot (version 0.9.5) ${ }^{66}$, ISOLDE (ChimeraX version 1.1/ISOLDE version 1.1) ${ }^{67}$, Refmac5 (version 5.8.0267) ${ }^{68}$ and MOE (version 2019.0102) (https://www.chemcomp.com), to arrive at the final models. For all complexes, epitopes on the RBD protein were determined by identifying all RBD residues within a 5.0 A distance from any Fab atoms. The analysis was performed using the MOE software package and the results were manually confirmed.

\section{Cryo-electron microscopy}

SARS-CoV-2 HexaPro $\mathrm{S}^{69}$ at $1.2 \mathrm{mg} \mathrm{ml}^{-1}$ was incubated with 1.2 -fold molar excess of recombinantly purified S2D106 or S2H 97 at $4{ }^{\circ} \mathrm{C}$ before application onto a freshly glow discharged 2.0/2.0 UltrAuFoil grid (200 mesh). Plunge freezing used a vitrobot MarkIV (Thermo Fisher Scien tific) using a blot force of 0 and 6.5 s blot time at $100 \%$ humidity and $23^{\circ} \mathrm{C}$.

For the S/S2D106 dataset, data were acquired using an FEI Titan Krios transmission electron microscope operated at $300 \mathrm{kV}$ and equipped with a Gatan K2 Summit direct detector and Gatan Quantum GIF energy filter, operated in zero-loss mode with a slit width of $20 \mathrm{eV}$. Automated data collection was carried out using Leginon ${ }^{70}$ at a nominal magnification of $130,000 \times$ with a pixel size of $0.525 \AA$. The dose rate was adjusted to 8 counts per pixel per $\mathrm{s}$, and each movie was acquired in super-resolution mode fractionated in 50 frames of $200 \mathrm{~ms}$. A total of 2,166 micrographs was collected, with a defocus range between -0.5 and $-2.5 \mu \mathrm{m}$. Movie frame alignment, estimation of the microscope contrast-transfer function parameters, particle picking, and extraction were carried out using Warp ${ }^{71}$. Particle images were extracted with a box size of 800 binned to 400 pixels $^{2}$ yielding a pixel size of $1.05 \AA$.

For theS/S2H97 dataset, data were acquired on an FEIGlaciostransmission electron microscope operated at $200 \mathrm{kV}$ equipped with a Gatan K2 Summit direct detector. Automated data collection was carried out using Leginon $^{70}$ at a nominal magnification of $36,000 \times$ with a pixel size of $1.16 \AA$. The dose rate was adjusted to 8 counts per pixel per s, and each movie was acquired in counting mode fractionated in 50 frames of $200 \mathrm{~ms}$. A total of 3,138 micrographs was collected in a single session with a defocus range comprised between -0.5 and $-3.0 \mu \mathrm{m}$. Preprocessing was performed using $\mathrm{Warp}^{71}$ and particle images were extracted with a box size of 400 pixels $^{2}$.

For the S/S2D106 and S/S2H97 datasets, two rounds of reference-free 2D classification were performed using CryoSPARC to select welldefined particle images ${ }^{72}$. These selected particles were subjected to two rounds of 3D classification with 50 iterations each (angular sampling $7.5^{\circ}$ for 25 iterations and $1.8^{\circ}$ with local search for 25 iterations), using our previously reported closed SARS-CoV-2S structure as initial mode $^{49}(\mathrm{PDB} 6 \mathrm{VXX})$ in Relion ${ }^{73}$.3D refinements were carried out using non-uniform refinement ${ }^{74}$ along with per-particle defocus refinement in CryoSPARC. Selected particle images were subjected to the Bayesian polishing procedure ${ }^{75}$ implemented in Relion3.0 before performing another round of non-uniform refinement in CryoSPARC followed by per-particle defocus refinement and again non-uniform refinement.

To further improve the density of the S2D106 Fab, the particles were then subjected to focus $3 \mathrm{D}$ classification without refining angles and shifts using a soft mask on RBD and Fab variable domains with a tau value of 60 in Relion. Particles belonging to classes with the best resolved local density were selected and subject to local refinement using CryoSPARC. Local resolution estimation, filtering, and sharpening were carried out using CryoSPARC. Reported resolutions are based on the gold-standard Fourier shell correlation (FSC) of 0.143 criterion and Fourier shell correlation curves were corrected for the effects of soft masking by high-resolution noise substitution ${ }^{76}$. UCSF $\mathrm{Chimera}^{77}$ and $\mathrm{Coot}^{78}$ were used to fit atomic models into the cryoEM maps. Spike-RBD/S2D106 Fab model was refined and relaxed using Rosetta using sharpened and unsharpened maps ${ }^{79}$.

\section{S2H97-induced spike refolding}

Ten micromolar native-like SARS-CoV-2 S was incubated with $13 \mu \mathrm{M}$ $\mathrm{S} 2 \mathrm{H} 97 \mathrm{Fab}$ for $1 \mathrm{~h}$ at room temperature. Samples were diluted to $0.01 \mathrm{mg} \mathrm{ml}^{-1}$ immediately before adsorption to glow-discharged carbon-coated copper grids for $\sim 30$ s before a $2 \%$ uranyl formate staining. Micrographs were recorded using the Leginon software ${ }^{70}$ on a $120 \mathrm{kV}$ FEI Tecnai G2 Spirit with a Gatan Ultrascan $40004 \mathrm{k} \times 4 \mathrm{k}$ CCD camera at $67,000 \times$ nominal magnification. The defocus ranged from -1.0 to $-2.0 \mu \mathrm{m}$ and the pixel size was $1.6 \AA$.

\section{Cell-surface antibody-mediated S1 shedding}

CHO-K1 cells stably expressing the prototypic SARS-CoV-2 spike protein were collected, washed in wash buffer (PBS, 1\% BSA, 2 mM EDTA) and 
resuspended in PBS. Ninety-thousand cells per well were dispensed into round bottom 96-well plates (Corning), and treated with $10 \mu \mathrm{g} \mathrm{ml}^{-1}$ TPCK-Trypsin (Worthington Biochem) for $30 \mathrm{~min}$ at $37^{\circ} \mathrm{C}$. Cells were washed and incubated with $15 \mu \mathrm{g} \mathrm{ml}^{-1}$ antibody across 5, 30, 60,120 and 180 min time points at $37^{\circ} \mathrm{C}$. Cells were washed with ice-cold wash buffer, and stained with $1.5 \mathrm{\mu g} \mathrm{ml}^{-1}$ Alexa Fluor647-conjugated goat anti-human IgG secondary antibody (Jackson Immunoresearch) for $30 \mathrm{~min}$ on ice in the dark. Cells were washed twice with cold wash buffer and analysed using a ZE5 cytometer (Bio-Rad) with acquisition chamber at $4{ }^{\circ} \mathrm{C}$. Binding at each time point was measured as mean fluorescence intensity (MFI), normalized to the MFI at the 5 min labelling time point. Data were analysed and plotted using GraphPad Prism v. 9.0.1.

\section{Cell-cell fusion of CHO-S cells}

Cell-cell fusion between S-expressing $\mathrm{CHO}-\mathrm{K} 1$ cells was performed as described by Lempp et al. ${ }^{31}$. $\mathrm{CHO}-\mathrm{K} 1$ cells stably expressing the prototypic SARS-CoV-2 spike protein were seeded in 96-well plates (Thermo Fisher Scientific) at 12,500 cells per well. The following day, antibody and nuclei marker Hoechst (final dilution 1:1,000) were added to cells and incubated for $24 \mathrm{~h}$. Cell-cell fusion was visualized using the Cytation 5 Imager (BioTek), and an object detection protocol was used to detect nuclei and measure their size. The nuclei of fused cells (syncytia) are aggregated at the centre of the syncytia and recognized as a uniquely large object that is gated according to its size. To quantify cell-cell fusion, we report the area of objects in fused cells divided by the total area of all objects, multiplied by $100 \%$ to represent it as a percentage.

\section{Antibody blockade of RBD binding to ACE2}

ACE2 blockade ELISA was performed as described by Piccoli et al. ${ }^{15}$. Unlabelled antibodies were serially diluted, mixed with RBD mouse Fc-tagged antigen (Sino Biological, final concentration $20 \mathrm{ng} \mathrm{ml}^{-1}$ ) and incubated for $30 \mathrm{~min}$ at $37^{\circ} \mathrm{C}$. The mix was added for $30 \mathrm{~min}$ to ELISA 96-well plates (Corning) pre-coated overnight at $4{ }^{\circ} \mathrm{C}$ with $2 \mathrm{\mu g} \mathrm{ml}^{-1}$ human ACE2 in PBS. Plates were washed and RBD binding was revealed using secondary goat anti-mouse IgG (Southern Biotech 1030-04). After washing, pNPP substrate was added and plates were read at $405 \mathrm{~nm}$. The percentage of inhibition was calculated as: $\left(1-\left(\mathrm{OD}_{\text {sample }}-\mathrm{OD}_{\text {neg ctrl }}\right) /\right.$ $\left.\left(\mathrm{OD}_{\text {pos ctrl }}-\mathrm{OD}_{\text {neg ctrl }}\right)\right) \times 100 \%$, where $\mathrm{OD}_{\text {sample }}, \mathrm{OD}_{\text {neg ctrl }}$ and $\mathrm{OD}_{\text {pos ctrl }}$ represent the optical densities of the sample, negative control and positive control, respectively.

\section{Inhibition of spike-mediated cell-cell fusion}

Cell-cell fusion inhibition assays were performed as described by McCallum et al. ${ }^{80}$. Vero E6 cells were seeded in 96-well plates at 15,000 cells per well in $70 \mu \mathrm{IDMEM}$ with high glucose and $2.4 \% \mathrm{FBS}$ (Hyclone). After $16 \mathrm{~h}$ at $37^{\circ} \mathrm{C}$ with $8 \% \mathrm{CO}_{2}$, the cells were transfected as follows: for 10 wells, $0.57 \mu$ g plasmid SARS-CoV-2-S-D19_pcDNA3.1 was mixed with $1.68 \mu \mathrm{l} \mathrm{X}$-tremeGENE HP in $30 \mu \mathrm{l}$ OPTIMEM. After 15 min incubation, the mixture was diluted 1:10 in DMEM medium and $30 \mu \mathrm{l}$ was added per well. Fourfold antibody serial dilutions were prepared and added to the cells, with a starting concentration of $20 \mu \mathrm{g} \mathrm{ml}^{-1}$. The following day, $30 \mu \mathrm{l} 5 \times$-concentrated DRAQ5 in DMEM was added per well and incubated for $2 \mathrm{~h}$ at $37^{\circ} \mathrm{C}$. Nine images of each well were acquired with a Cytation 5 equipment for analysis.

\section{S2H97 prophylactic protection in Syrian hamsters}

We used a validated SARS-CoV-2 Syrian golden hamster model of infection $^{81,82}$ to test $\mathrm{S} 2 \mathrm{H} 97$ prophylactic efficacy. Experiments were performed in the high-containment $\mathrm{A} 3$ and BSL3+ facilities of the KU Leuven Rega Institute (3CAPS) under licenses AMV 30112018 SBB 219 20180892 and AMV 23102017 SBB 21920170589 according to institutional guidelines.

Syrian hamsters (Mesocricetus auratus) were purchased from Janvier Laboratories. Hamsters were housed per two in ventilated isolator cages
(IsoCage N Biocontainment System, Tecniplast) with ad libitum access to food, water, and cage enrichment (wood block). Housing conditions and experimental procedures were approved by the ethical committee of animal experimentation of KU Leuven (license P065-2020). Sample sizes of 6 hamsters was determined in order to have a significant difference of at least $1 \log ($ viral RNA level) (effect size $d=2.004$ ) between control and treatment groups, by using a two-tailed $t$-test with $80 \%$ power and an alpha of 0.05 , calculated with $G^{*}$ Power 3.1 software. Sixto ten-week-old female hamsters were randomized for administration of $25 \mathrm{mg} \mathrm{kg}^{-1} \mathrm{~S} 2 \mathrm{H} 97$ antibody or $20 \mathrm{mg} \mathrm{kg}^{-1}$ human isotype control via intraperitoneal injection. Approximately $5 \mathrm{~h}$ before infection, animals were anesthetized with isoflurane to allow collection of a blood sample from the jugular vein to be used for antibody quantification. Forty-eight hours after antibody injection, hamsters were infected intranasally with $1.89 \times 10^{6} \mathrm{TCID}_{50}$ SARS-CoV-2 virus in $50 \mu$ inoculum. The challenge virus was a SARS-CoV-2 Wuhan isolate from February, 2020 (EPI_ISL_407976), passaged on Vero E6 cells. Passage 6 stock titre was determined by end-point dilution on Vero E6 cells by the Reed and Muench method ${ }^{83}$, expressed as $50 \%$ tissue culture infectious dose $\left(\mathrm{TCID}_{50}\right)$.

Hamsters were monitored for appearance, behaviour, and weight. At day 4 post-infection, hamsters were euthanized by intraperitoneal injection of $500 \mu \mathrm{l}$ Dolethal $\left(200 \mathrm{mg} \mathrm{ml}^{-1}\right.$ sodium pentobarbital, Vétoquinol SA). Lungs were collected, homogenized via bead disruption (Precellys) in $350 \mu \mathrm{l} \mathrm{RLT}$ buffer (RNeasy Mini kit, Qiagen) and centrifuged $\left(10,000 \mathrm{rpm}, 5 \mathrm{~min}, 4^{\circ} \mathrm{C}\right)$ to pellet cell debris. RNA was extracted using a NucleoSpin kit (Macherey-Nagel) according to manufacturer instructions. Quantitative PCR with reverse transcription (RT-qPCR) was performed on a LightCycler96 platform (Roche) using the iTaq Universal Probes One-Step RT-qPCR kit (Bio-Rad) with N2 primers and probes targeting the nucleocapsid ${ }^{81}$. Standards of SARS-CoV-2 cDNA (IDT) were used to express viral genome copies per mg tissue. To quantify infectious SARS-CoV-2 particles, end-point titrations were performed on confluent Vero E6 cells in 96-well plates. Viral titres were calculated as above, and were expressed as $\mathrm{TCID}_{50}$ per mg tissue. The circulating antibody levels were measured by mesoscale bridging ELISA, using an anti-human LS mutation monoclonal antibody as a capture and anti-human $\mathrm{CH} 2$ monoclonal antibody as detection. Technicians performing RNA, virus, and antibody quantification were blinded to the treatment groups of processed samples. RNA and viral levels were compared between treatment and control via two-tailed Mann-Whitney test, excluding the two treatment animals with undetectable S2H97 levels at time of viral challenge.

\section{Blockade of binding serology competition assays}

Sera blockade of antibody binding was performed as described in Piccoli et al. ${ }^{15}$. In brief, human IgG1 antibodies were biotinylated using the EZ-link NHS-PEO solid phase biotinylation kit (Pierce). Each labelled antibody was tested for binding to RBD by ELISA, and a concentration for each antibody competition experiment was selected to achieve $80 \%$ maximal binding $\left(\mathrm{EC}_{80}\right)$. ELISA 96-well plates (Corning) were pre-coated overnight at $4{ }^{\circ} \mathrm{C}$ with $1 \mu \mathrm{g} \mathrm{ml}^{-1}$ of mouse Fc-tagged RBD antigen (Sino Biological) in PBS. Unlabelled sera or plasma were serially diluted and added to ELISA plates for $30 \mathrm{~min}$, followed by addition of biotinylated anti-RBD antibody at its $\mathrm{EC}_{80}$ concentration. After 30 min incubation, plates were washed and antibody binding was detected using alkaline phosphatase-conjugated streptavidin (Jackson ImmunoResearch). Plates were washed, pNPP substrate (Sigma-Aldrich) was added, and plates were read at $405 \mathrm{~nm}$. The percentage of inhibition of antibody binding was calculated as: $\left(1-\left(\mathrm{OD}_{\text {sample }}-\mathrm{OD}_{\text {negctrl }}\right) /\left(\mathrm{OD}_{\text {posctrl }}-\mathrm{OD}_{\text {neg ctrl }}\right)\right)$ $\times 100$.

\section{Selection of VSV-SARS-CoV-2 monoclonal antibody resistance mutants}

VSV-SARS-CoV-2S chimera was used to select for SARS-CoV-2S monoclonal antibody resistant mutants (MARMs) as previously described ${ }^{1,84}$. 
In brief, MARMs were recovered by plaque isolation on Vero E6 cells (ATCC, CRL-1586) with the indicated monoclonal antibody in the overlay. The concentration of monoclonal antibody in the overlay was determined by neutralization assays at a MOI of 100. Escape clones were plaque-purified on Vero cells (ATCC, CCL-81) in the presence of monoclonal antibody, and plaques in agarose plugs were amplified on MA104 cells (a gift from H. B. Greenberg (Stanford School of Medicine)) with the monoclonal antibody present in the medium. Viral stocks were amplified on MA104 cells at an MOI of 0.01 in Medium 199 containing $2 \%$ FBS and $20 \mathrm{mM}$ HEPES pH 7.7 (Millipore Sigma) at $34{ }^{\circ} \mathrm{C}$. Viral supernatants were collected upon extensive cytopathic effect and clarified of cell debris by centrifugation at $1,000 \mathrm{~g}$ for $5 \mathrm{~min}$. Aliquots were maintained at $-80^{\circ} \mathrm{C}$. Viral RNA was extracted from VSV-SARS-CoV-2 mutant viruses using RNeasy Mini kit (Qiagen), and S was amplified using OneStep RT-PCR Kit (Qiagen). The mutations were identified by Sanger sequencing (Genewiz). Their resistance was verified by subsequent virus infection in the presence or absence of antibody. In brief, Vero cells were seeded into 12-well plates for overnight. The virus was serially diluted using DMEM and cells were infected at $37^{\circ} \mathrm{C}$ for $1 \mathrm{~h}$. Cells were cultured with an agarose overlay in the presence or absence of monoclonal antibody at $34^{\circ} \mathrm{C}$ for 2 days. Plates were scanned on a biomolecular imager and expression of eGFP is shown at $48 \mathrm{~h}$ after infection. The S2X58-selected mutation S494L is not shown in Fig. 3a, as its effect on RBD expression was below the deep mutational scanning computational filter.

\section{Viral replication fitness assays}

Vero E6 cells (ATCC, CRL-1586) were seeded at $1 \times 10^{6}$ cells per well in 6-well plates. Cells were infected with MOI of 0.02 , with wild-type and four mutant VSV-SARS-CoV-2S chimeras mixed at equal $(0.20)$ frequencies. Following $1 \mathrm{~h}$ incubation, cell monolayers were washed 3 times with HBBS and cultures were incubated for $72 \mathrm{~h}$ in humidified incubators at $34^{\circ} \mathrm{C}$. To passage the progeny viruses, virus mixture was continuously passaged 4 times in Vero E6 cells at MOI of 0.02. Cellular RNA samples from each passages were extracted using RNeasy Mini kit (QIAGEN) and subjected to next-generation sequencing as described previously to confirm the introduction and frequency of substitutions ${ }^{84}$.

\section{Molecular dynamics simulations}

Full details of molecular dynamics workflow and analysis are available on GitHub: https://github.com/choderalab/rbd-ab-contact-analysis. The RBD-S309 complex was constructed from PDB 7JX3 (chains A, $B$ and $R$ ). 7JX3 was first refined using ISOLDE ${ }^{67}$. Refinement included adjusting several rotamers, flipping several peptide bonds, fixing several weakly resolved waters, and building in a missing four-residue-long loop. Though the N343 glycan $N$-Acetylglucosamine (NAG) was present in 7JX3, ISOLDE was used to construct a complex glycan at N343. The full glycosylation pattern was determined from refs. ${ }^{85,86}$. The glycan structure used for N343 (FA2G2) corresponds to the most stable conformer obtained from multi microsecond molecular dynamics simulations of cumulative sampling ${ }^{87}$. The base NAG residue in FA2G 2 was aligned to the corresponding NAG stub in the RBD-S309 model and any resulting clashes were refined in ISOLDE. The same process was repeated for the RBD-S2H97 crystal structure.

The refined glycosylated RBD-S309 and RBD-S2H97 complexes were prepared for simulation using tleap from AmberTools $20^{88}$. All relevant disulfide bridges and covalent connections in glycan structures were specified. The glycosylated proteins were parameterized with the Amber ff $14 \mathrm{SB}^{89}$ and GLYCAM_06j-1 ${ }^{90}$ force fields. The systems were solvated using the TIP3P rigid water model ${ }^{91}$ in a truncated octahedral box with $2.2 \mathrm{~nm}$ solvent padding on all sides. The shape and size of the solvent box were chosen to prevent the protein complex from interacting with its periodic image. The solvated systems were then neutralized with $0.15 \mathrm{M} \mathrm{NaCl}$ using the Li/Merz ion parameters of monovalent ions for the TIP3P water model (12-6 normal usage set $)^{92}$. Virtual bonds were added across chains that should be imaged together to aid the post-processing of trajectories.

Each system was energy-minimized with an energy tolerance of $10 \mathrm{~kJ} \mathrm{~mol}^{-1}$ and equilibrated five times independently using the OpenMMTools 0.20.0 (https://github.com/choderalab/openmmtools) BAOAB Langevin integrator ${ }^{93}$ for $20 \mathrm{~ns}$ in the NPT ( $\left.P=1 \mathrm{~atm}, T=310 \mathrm{~K}\right)$ ensemble with a time step of $4.0 \mathrm{fs}$, a collision rate of $1.0 \mathrm{ps}^{-1}$, and a relative constraint tolerance of $1 \times 10^{-5}$. Hydrogen atom masses were set to $4.0 \mathrm{amu}$ by transferring mass from connected heavy atoms, bonds to hydrogen were constrained, and centre of mass motion was not removed. Pressure was controlled by a molecular-scaling Monte Carlo barostat with an update interval of 25 steps. Non-bonded interactions were treated with the Particle Mesh Ewald method ${ }^{94}$ using a real-space cut-off of $1.0 \mathrm{~nm}$ and the OpenMM default relative error tolerance of 0.0005 , with grid spacing selected automatically. The simulations were subsequently packaged to seed for production simulation on Folding@home $\mathrm{e}^{95,96}$. Default parameters were used unless noted otherwise.

The equilibrated structures (five per complex) were used to initiate parallel distributed molecular dynamics simulations on Folding@ home ${ }^{95,96}$. Simulations were run with OpenMM 7.4.2 (compiled into Folding@home core22 0.0.13).Production simulations used the same Langevin integrator as the NPT equilibration described above. A total of 5,000 and 4,985 independent molecular dynamics simulations were generated on Folding@home for RBD-S309 and RBD-S2H97, respectively. Conformational snapshots (frames) were stored at an interval of $1 \mathrm{~ns}$ per frame for subsequent analysis. The final datasets contained $1.1 \mathrm{~ms}$ and $623.7 \mu \mathrm{s}$ of aggregate simulation time for RBD-S309 and RBD-S2H97, respectively. This trajectory dataset (without solvent) are available at the MoISSI COVID-19 molecular structure and therapeutics hub (https://covid.molssi.org//simulations/\#foldinghome-simulatio ns-of-the-sars-cov-2-spike-rbd-bound-to-monoclonal-antibody-s309 and https://covid.molssi.org//simulations/\#foldinghome-simulations -of-the-sars-cov-2-spike-rbd-bound-to-monoclonal-antibody-s2h97).

The first 100 ns of each trajectory was discarded (to allow relaxation away from the crystal structure), yielding total simulation times of 644.3 and $262.9 \mu$ s used for analysis of RBD-S309 and RBD-S2H97 systems, respectively. All trajectories had solute structures aligned to their first frame and centred using MDTraj ${ }^{97}$. Residues were considered to be at the interface if they were within $10 \AA$ of any antibody Fab/RBD residue (with the exception of RBD N343 glycans, where all glycan residues were considered). The minimum distance of heavy atoms between every pair of interface residues was computed for every frame (1 ns) using MDAnalysis ${ }^{98,99}$. A close contact was counted if the minimum distance between a residue pair was below $3.5 \AA$ (if one of the residues was hydrophobic, a $4.5 \AA$ A cut-off was used). The contribution of each RBD residue to close contacts was calculated as a percentage by summation of the number of close contacts for a particular RBD residue and normalizing by the total number of close contact interactions over all frames of each simulation.

\section{Materials availability}

The SARS-CoV-2 RBD mutant libraries (\#1000000172) and unmutated parental plasmid (\#166782) are available on Addgene. Other materials generated in this study will be made available on request and may require a material transfer agreement.

\section{Reporting summary}

Further information on research design is available in the Nature Research Reporting Summary linked to this paper.

\section{Data availability}

Interactive escape maps and structural visualizations can be found at https://jbloomlab.github.io/SARS-CoV-2-RBD_MAP_Vir_mAbs/.Raw Illumina sequencing data from deep mutational scanning experiments 
are available on NCBI Sequence Read Archive BioSample under accessions SAMN18315604 (SARS-CoV-2 mutant selection data) and SAMN18316011(sarbecovirus RBD selection data). PacBio sequencing data used to link N16 barcodes to sarbecovirus RBD variant are available on NCBI SRA BioSample under accession SAMN18316101. A complete table of deep mutational scanning antibody escape fractions is provided on GitHub: https://github.com/jbloomlab/SARS-CoV-2-RBD_ MAP_Vir_mAbs/blob/main/results/supp_data/all_antibodies_raw_data. csv. This table includes both antibodies first described in this study (Fig. 1b, c), and all other antibody selections that were re-processed to generate Fig. 4a. Coordinates and structure factors have been deposited in the Protein Data Bank (PDB) with accession code 7R6W for RBD-S2X35-S309, 7M7W for RBD-S2H97-S2X259 and 7R6X for RBD-S2E12-S304-S309. Cryo-electron microscopy structure data and models are available from the Electron Microscopy Data Bank (EMDB) under accession codes EMD-24300 for S/S2D106, EMDB EMD-24299 and PDB 7R7N for the S/S2D106 local refinement, and EMDB EMD-24301 for $\mathrm{S} / \mathrm{S} 2 \mathrm{H} 97$. The raw and processed molecular dynamics trajectory data are available at the MolSSI COVID-19 Molecular Structure and Therapeutics Hub:https://covid.molssi.org//simulations/\#foldinghome-simulationsof-the-sars-cov-2-spike-rbd-bound-to-monoclonal-antibody-s309 and https://covid.molssi.org//simulations/\#foldinghome-simulations-ofthe-sars-cov-2-spike-rbd-bound-to-monoclonal-antibody-s2h97. All other datasets generated during and/or analysed during the current study are available from the corresponding author on reasonable request. Source data are provided with this paper.

\section{Code availability}

A repository containing all code, analysis and summary notebooks for the analysis of the SARS-CoV-2 deep mutational scanning escape selections is available on GitHub at https://github.com/jbloomlab/ SARS-CoV-2-RBD_MAP_Vir_mAbs. A repository containing code and analysis of the sarbecovirus RBD library binding experiments is available on GitHub at https://github.com/jbloomlab/SARSr-CoV_RBD_MAP. A repository containing code and analysis of molecular dynamics simulations is available on GitHub at https://github.com/choderalab/ rbd-ab-contact-analysis.

43. Crawford, K. H. D. et al. Protocol and reagents for pseudotyping lentiviral particles with SARS-CoV-2 spike protein for neutralization assays. Viruses 12, 513 (2020).

44. Xie, X. et al. A nanoluciferase SARS-CoV-2 for rapid neutralization testing and screening of anti-infective drugs for COVID-19. Nat. Commun. 11, 5214 (2020).

45. Takada, A. et al. A system for functional analysis of Ebola virus glycoprotein. Proc. Natl Acad. Sci. USA 94, 14764-14769 (1997).

46. Riblett, A. M. et al. A haploid genetic screen identifies heparan sulfate proteoglycans supporting rift valley fever virus infection. J. Virol. 90, 1414-1423 (2015).

47. Giroglou, T. et al. Retroviral vectors pseudotyped with severe acute respiratory syndrome coronavirus S protein. J. Virol. 78, 9007-9015 (2004).

48. Collier, D. A. et al. Sensitivity of SARS-CoV-2 B.1.1.7 to mRNA vaccine-elicited antibodies. Nature 593, 136-141 (2021).

49. Walls, A. C. et al. Structure, function, and antigenicity of the SARS-CoV-2 spike glycoprotein. Cell 181, 281-292.e6 (2020).

50. Otwinowski, J., McCandlish, D. M. \& Plotkin, J. B. Inferring the shape of global epistasis Proc. Natl Acad. Sci. USA 115, E7550-E7558 (2018).

51. Hilton, S. K. et al. dms-view: Interactive visualization tool for deep mutational scanning data. J. Open Source Softw. 5, 2353 (2020).

52. Song, H.-D. et al. Cross-host evolution of severe acute respiratory syndrome coronavirus in palm civet and human. Proc. Natl Acad. Sci. USA 102, 2430-2435 (2005).

53. Tong, S. et al. Detection of novel SARS-like and other coronaviruses in bats from Kenya. Emerg. Infect. Dis. 15, 482-485 (2009).

54. Zhou, H. et al. A novel bat coronavirus closely related to SARS-CoV-2 contains natural insertions at the S1/S2 cleavage site of the spike protein. Curr. Biol. 30, 2196-2203.e3 (2020).

55. Lam, T. T.-Y. et al. Identifying SARS-CoV-2-related coronaviruses in Malayan pangolins. Nature 583, 282-285 (2020).

56. Katoh, K. \& Standley, D. M. MAFFT multiple sequence alignment software version 7: improvements in performance and usability. Mol. Biol. Evol. 30, 772-780 (2013).

57. Suyama, M., Torrents, D. \& Bork, P. PAL2NAL: robust conversion of protein sequence alignments into the corresponding codon alignments. Nucleic Acids Res. 34, W609-W612 (2006).

58. Stamatakis, A. RAxML version 8: a tool for phylogenetic analysis and post-analysis of large phylogenies. Bioinformatics 30, 1312-1313 (2014).
59. Elbe, S. \& Buckland-Merrett, G. Data, disease and diplomacy: GISAID's innovative contribution to global health. Glob. Chall. 1, 33-46 (2017).

60. Lan, J. et al. Structure of the SARS-CoV-2 spike receptor-binding domain bound to the ACE2 receptor. Nature 581, 215-220 (2020).

61. Yuan, M. et al. A highly conserved cryptic epitope in the receptor binding domains of SARS-CoV-2 and SARS-CoV. Science 368, 630-633 (2020).

62. Hansen, J. et al. Studies in humanized mice and convalescent humans yield a SARS-CoV-2 antibody cocktail. Science 369, 1010-1014 (2020).

63. Shi, R. et al. A human neutralizing antibody targets the receptor-binding site of SARS-CoV-2. Nature 584, 120-124 (2020).

64. Kabsch, W. XDS. Acta Crystallogr. D 66, 125-132 (2010).

65. McCoy, A. J. et al. Phaser crystallographic software. J. Appl. Cryst. 40, 658-674 (2007).

66. Emsley, P., Lohkamp, B., Scott, W. G. \& Cowtan, K. Features and development of Coot. Acta Crystallogr. D 66, 486-501 (2010).

67. Croll, T. I. ISOLDE: a physically realistic environment for model building into low-resolution electron-density maps. Acta Crystallogr. D 74, 519-530 (2018).

68. Murshudov, G. N. et al. REFMAC5 for the refinement of macromolecular crystal structures. Acta Crystallogr. D 67, 355-367 (2011).

69. Hsieh, C.-L. et al. Structure-based design of prefusion-stabilized SARS-CoV-2 spikes. Science 369, 1501-1505 (2020).

70. Suloway, C. et al. Automated molecular microscopy: the new Leginon system. J. Struct. Biol. 151, 41-60 (2005).

71. Tegunov, D. \& Cramer, P. Real-time cryo-electron microscopy data preprocessing with Warp. Nat. Methods 16, 1146-1152 (2019).

72. Punjani, A., Rubinstein, J. L., Fleet, D. J. \& Brubaker, M. A. cryoSPARC: algorithms for rapid unsupervised cryo-EM structure determination. Nat. Methods 14, 290-296 (2017).

73. Zivanov, J. et al. New tools for automated high-resolution cryo-EM structure determination in RELION-3. eLife 7, e42166 (2018).

74. Punjani, A., Zhang, H. \& Fleet, D. J. Non-uniform refinement: adaptive regularization improves single-particle cryo-EM reconstruction. Nat. Methods 17, 1214-1221 (2020).

75. Zivanov, J., Nakane, T. \& Scheres, S. H. W. A Bayesian approach to beam-induced motion correction in cryo-EM single-particle analysis. IUCrJ 6, 5-17 (2019).

76. Ramlaul, K., Palmer, C. M., Nakane, T. \& Aylett, C. H. S. Mitigating local over-fitting during single particle reconstruction with SIDESPLITTER. J. Struct. Biol. 211, 107545 (2020).

77. Pettersen, E. F. et al. UCSF ChimeraX: structure visualization for researchers, educators, and developers. Protein Sci. 30, 70-82 (2021).

78. Casañal, A., Lohkamp, B. \& Emsley, P. Current developments in Coot for macromolecular model building of electron cryo-microscopy and crystallographic data. Protein Sci. 29, 1069-1078 (2020).

79. Wang, R. Y.-R. et al. Automated structure refinement of macromolecular assemblies from cryo-EM maps using Rosetta. eLife 5, e17219 (2016).

80. McCallum, M. et al. N-terminal domain antigenic mapping reveals a site of vulnerability for SARS-CoV-2. Cell 184, 2332-2347.e16 (2021).

81. Boudewijns, R. et al. STAT2 signaling restricts viral dissemination but drives severe pneumonia in SARS-CoV-2 infected hamsters. Nat. Commun. 11, 5838 (2O2O).

82. Sanchez-Felipe, L. et al. A single-dose live-attenuated YF17D-vectored SARS-CoV-2 vaccine candidate. Nature 590, 320-325 (2021)

83. Reed, L. J. \& Muench, H. A simple method of estimating fifty percent endpoints. Am. J. Epidemiol. 27, 493-497 (1938).

84. Case, J. B. et al. Neutralizing antibody and soluble ACE2 inhibition of a replicationcompetent VSV-SARS-CoV-2 and a clinical isolate of SARS-CoV-2. Cell Host Microbe 28, 475-485.e5 (2020)

85. Shajahan, A. et al. Comprehensive characterization of $\mathrm{N}$ - and $\mathrm{O}$-glycosylation of SARS-CoV-2 human receptor angiotensin converting enzyme 2. Glycobiology 31, 410-424 (2020).

86. Watanabe, Y., Allen, J. D., Wrapp, D., McLellan, J. S. \& Crispin, M. Site-specific glycan analysis of the SARS-CoV-2 spike. Science 369, 330-333 (2020).

87. Harbison, A. M., Brosnan, L. P., Fenlon, K. \& Fadda, E. Sequence-to-structure dependence of isolated IgG Fc complex biantennary N-glycans: a molecular dynamics study. Glycobiology 29, 94-103 (2019).

88. Case, D.A. et al. Amber 2020 (Univ. of California, 2020).

89. Maier, J. A. et al. Ff14SB: improving the accuracy of protein side chain and backbone parameters from ff99SB. J. Chem. Theory Comput. 11, 3696-3713 (2015)

90. Kirschner, K. N. et al. GLYCAMO6: a generalizable biomolecular force field. Carbohydrates. J. Comput. Chem. 29, 622-655 (2008).

91. Jorgensen, W. L., Chandrasekhar, J., Madura, J. D., Impey, R. W. \& Klein, M. L. Comparison of simple potential functions for simulating liquid water. J. Chem. Phys. 79, 926-935 (1983).

92. Li, P., Song, L. F. \& Merz, K. M., Jr Systematic parameterization of monovalent ions employing the nonbonded model. J. Chem. Theory Comput. 11, 1645-1657 (2015).

93. Leimkuhler, B. \& Matthews, C. Robust and efficient configurational molecular sampling via Langevin dynamics. J. Chem. Phys. 138, 174102 (2013).

94. Darden, T., York, D. \& Pedersen, L. Particle mesh Ewald: an $N \cdot \log (N)$ method for Ewald sums in large systems. J. Chem. Phys. 98, 10089-10092 (1993).

95. Shirts, M. \& Pande, V. S. COMPUTING: Screen savers of the world unite! Science 290, 1903-1904 (2000).

96. Zimmerman, M. I. et al. SARS-CoV-2 simulations go exascale to predict dramatic spike opening and cryptic pockets across the proteome. Nat. Chem. 13, 651-659 (2021).

97. McGibbon, R. T. et al. MDTraj: a modern open library for the analysis of molecular dynamics trajectories. Biophys. J. 109, 1528-1532 (2015).

98. Michaud-Agrawal, N., Denning, E. J., Woolf, T. B. \& Beckstein, O. MDAnalysis: a toolkit for the analysis of molecular dynamics simulations. J. Comput. Chem. 32, 2319-2327 (2011).

99. Gowers, R. et al. MDAnalysis: A Python package for the rapid analysis of molecular dynamics simulations. in Proc. 15th Python in Science Conference (SciPy, 2016). 


\section{Article}

Acknowledgements We thank I. Hoffman for assistance in refinement of crystal structures, G. R. Bowman, J. Coffland and P. K. Eastman for developing and maintaining the Folding@ home infrastructure, Amazon Web Services for Folding@home infrastructure support, the Folding@home volunteers who contributed their computational resources to this project (FAH Project 17336-17340), R. P. Wiewiora and S. Singh for their assistance with Folding@home, and A. M. Harbison and E. Fadda for assistance in glycan modeling. This work was supported by the Fred Hutch Flow Cytometry and Genomics facilities, the Fred Hutch Scientific Computing group supported by ORIP grant S10OD028685, and the University of Washington Arnold and Mabel Beckman Cryo-EM Center. This work was supported by the NIH/NIAID (R01Al127893 and R01Al141707 to J.D.B., DP1Al158186 and HHSN272201700059C to D.V. and T32AI083203 to A.J.G.), the NIH/NIGMS (R01GM120553 to D.V., and grant R01GM121505 and R01GM132386 to J.D.C.), the $\mathrm{NIH} / \mathrm{NCl}$ (P3OCA008748 to J.D.C.), the National Science Foundation (NSF CHI1904822 to J.D.C.), the Damon Runyon Cancer Research Foundation (T.N.S.), the Gates Foundation (INV-004949 to J.D.B.), a Pew Biomedical Scholars Award (D.V.), Investigators in the Pathogenesis of Infectious Disease Awards from the Burroughs Wellcome Fund (J.D.B. and D.V.), the Wellcome Trust (209407/Z/17/Z to T.I.C.), Fast Grants (D.V.), Bayer (W.G.G.) and the Molecular Sciences Software Institute (I.Z.). J.D.B. is an Investigator of the Howard Hughes Medical Institute. The Molecular Biology Consortium beamline 4.2.2 of the Advanced Light Source, a US DOE Office of Science User Facility under contract no. DE-AC02-05CH11231, is supported in part by the ALS-ENABLE program funded by the NIH/NIGMS (P3OGM124169-01). Use of the Stanford Synchrotron Radiation Lightsource, SLAC National Accelerator Laboratory, is supported by the US Department of Energy, Office of Science, Office of Basic Energy Sciences under contract no. DE-AC02-76SF00515. The SSRL Structural Molecular Biology Program is supported by the DOE Office of Biological and Environmental Research, and by the $\mathrm{NIH/NIGMS} \mathrm{(P30GM133894).}$

Author contributions Conceived research and designed study: T.N.S., N.C., H.W.V., D.C., J.D.B. and G.S. Antibody discovery: F.Z., D.P., M.B., R.M., A.D.M., E.C., M.S.P. and D.C. Expression and purification of proteins: N.C., P.H., G.L., N.S., J.E.B., A.C.W., K.C., S.J. and M.M. Antibody functional experiments: Y.-J.P., Z.L., F.Z., D.P., M.B., R.M., J.E.B., M.A.T., A.C.W., J.A.W., A.D.M., L.E.R., J.Z., M.M.-R., H.K., J.R.D., H.T., J.B., C.S.-F., M.P.H., M.A., E.D.Jr, S.S., C.H.-D., L.P., F.B., F.A.L. and S.P.J.W. Deep mutational scanning experiments and analysis: T.N.S., A.A., A.J.G. and A.S.D. Hamster model: R.A., S.-Y.C.F., F.B. and J.N. Bioinformatics analysis: J.d.I. and A.T. Structure determination N.C., Y.-J.P., P.H., J.E.B., T.I.C., J.C.N., D.V. and G.S. Molecular dynamics simulation and analysis:
W.G.G., I.Z. and J.D.C. Supervision: M.S.P., J.D.C., C.M.H., S.P.J.W., D.V., D.C., J.D.B. and G.S. Writing of the initial draft: T.N.S., N.C., D.C., J.D.B. and G.S. Editing of the final version: all authors.

Competing interests N.C., F.Z., D.P., M.B., P.H., R.M., J.A.W., A.D.M., L.E.R., J.Z., M.M.-R., H.K., J.R.D., H.T., J.B., C.S.-F., M.P.H., J.d.I., G.L., M.A., N.S., K.C., S.J., M.M., E.D.Jr, E.C., C.H.-D., L.P., F.B., A.T., F.A.L., M.S.P., C.M.H., H.W.V., D.C. and G.S. are or were employees of Vir Biotechnology and may hold shares in Vir Biotechnology. D.C. is currently listed as an inventor on multiple patent applications, which disclose the subject matter described in this manuscript. After the submission of the initial version of this study, J.D.B. began consulting for Moderna on viral evolution and epidemiology. J.D.B. has the potential to receive a share of IP revenue as an inventor on a Fred Hutchinson Cancer Research Center-optioned technology/patent (application WO2O20006494) related to deep mutational scanning of viral proteins. H.W.V. is a founder of PierianDx and Casma Therapeutics. Neither company provided funding for this work nor is performing related work. J.C.N., T.I.C. and D.V. are consultants for Vir Biotechnology Inc. The Veesler laboratory has received a sponsored research agreement from Vir Biotechnology Inc. J.D.C. is a current member of the Scientific Advisory Boards of OpenEye Scientific Software, Interline Therapeutics, and Redesign Science. The Chodera laboratory receives or has received funding from the National Institute of Health, the National Science Foundation, the Parker Institute for Cancer Immunotherapy, Relay Therapeutics, Entasis Therapeutics, Silicon Therapeutics, EMD Serono (Merck KGaA), AstraZeneca, Vir Biotechnology, XtalPi, Interline Therapeutics, and the Molecular Sciences Software Institute, the Starr Cancer Consortium, the Open Force Field Consortium, Cycle for Survival, a Louis V. Gerstner Young Investigator Award, and the Sloan Kettering Institute. A complete funding history for the Chodera lab can be found at http://choderalab.org/funding. The other authors declare no competing interests.

Additional information

Supplementary information The online version contains supplementary material available at https://doi.org/10.1038/s41586-021-03807-6.

Correspondence and requests for materials should be addressed to D.C., J.D.B. or G.S.

Peer review information Nature thanks the anonymous reviewers for their contribution to the peer review of this work.

Reprints and permissions information is available at http://www.nature.com/reprints 
a

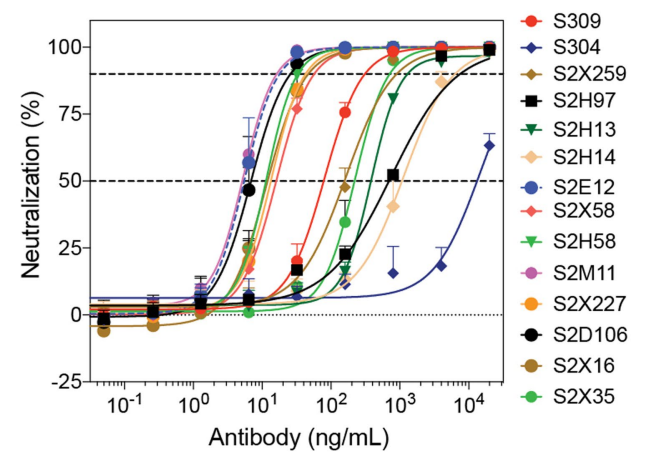

C

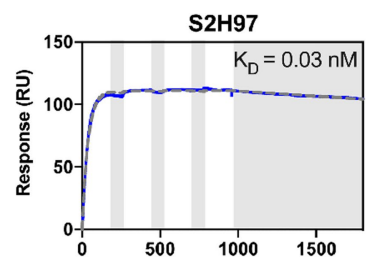

S2D106

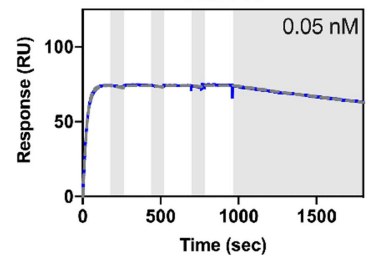

$\mathrm{S} 2 \times 58$ b

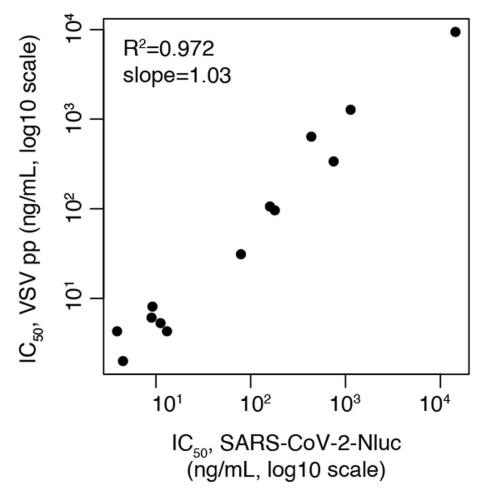

d
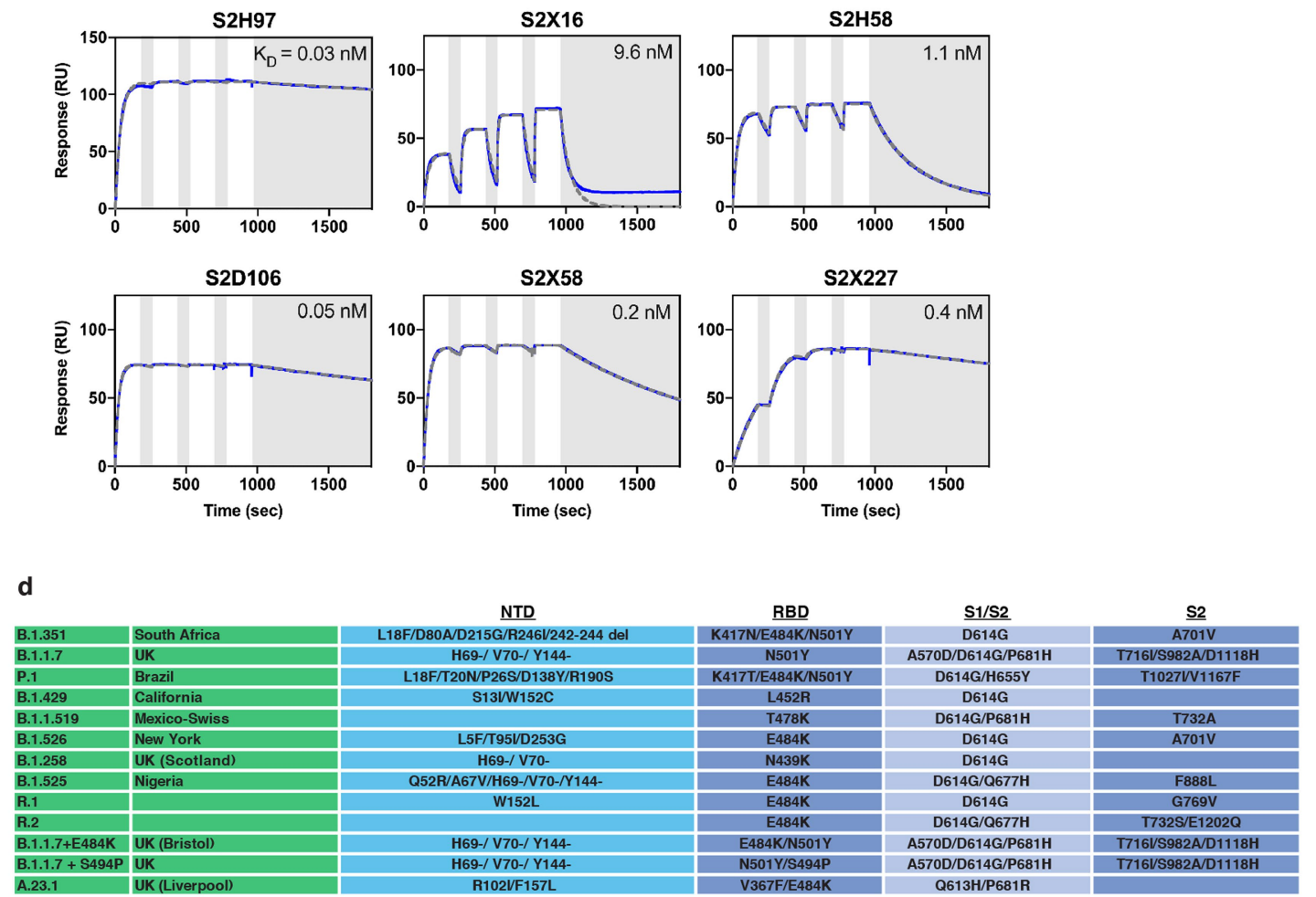

Extended Data Fig. $1 \mid$ Antibody neutralization and binding data.

a, Neutralization of authentic SARS-CoV-2 (SARS-CoV-2-Nluc) by 14 antibodies. Shown are representative live virus neutralization plots, measured with entry into Vero E6 cells. Symbols are means \pm s.d. of technical triplicates. Dashed lines indicate $\mathrm{IC}_{50}$ and $\mathrm{IC}_{90}$ values. All antibodies were measured at each concentration point in the series, with hidden points due to overplotting reflecting overlap at the upper and lower neutralization limits. b. Correlation in antibody neutralization $\mathrm{IC}_{50}$ as determined in spike-pseudotyped VSV particles ( $n=3$ to 8$)$ versus authentic SARS-CoV-2 $(n=3)$. c, Representative SPR sensorgrams of Fab fragments of the six newly described antibodies binding to the SARS-CoV-2 RBD (Fig.1a gives average values for $n=2-4$ measurements). White and grey stripes indicate association and dissociation phases, respectively. Data shown in blue and fit to binding model shown in dashed gray line. Binding affinities for previously described antibodies shown in Fig. 1a are consistent with measurements from Piccoli et al. (S304, S309, S2X35, S2H13, $\mathrm{S} 2 \mathrm{H} 14)^{15}$ and Tortorici et al. (S2E12) ${ }^{8}$. d, Identifiers and spike genotypes of SARS-CoV-2 variants tested in neutralization assays in Figs. 2d, 3b. 
a

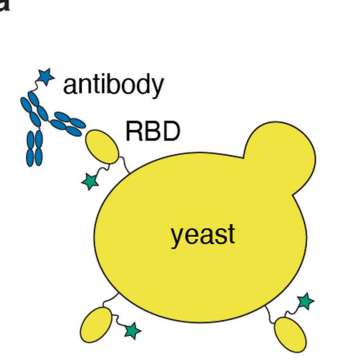

b

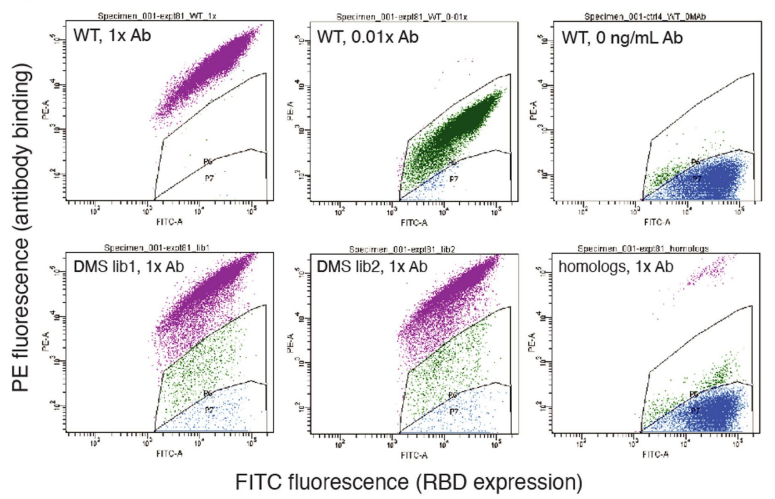

SARS-CoV-2 RBD library

containing all mutations compatible with RBD folding and low-affinity ACE2 binding

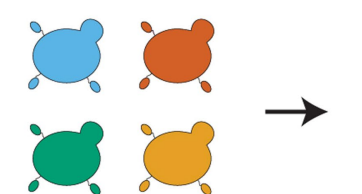

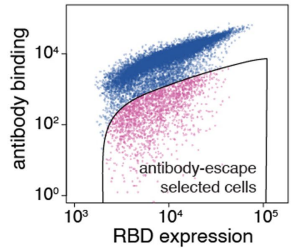

\begin{tabular}{crrrc}
\hline antibody & $\begin{array}{c}\text { \% escape } \\
\text { DMS lib1 }\end{array}$ & $\begin{array}{c}\text { \% escape } \\
\text { DMS lib2 }\end{array}$ & $\begin{array}{c}\text { \% escape } \\
\text { homolog lib }\end{array}$ & $\begin{array}{c}\text { \% full escape } \\
\text { homolog lib* }\end{array}$ \\
\hline S309 & 10.0 & 19.9 & 49.0 & \\
S2H97 & 6.9 & 8.1 & 5.6 & 0.7 \\
S304 & 12.3 & 15.5 & 41.2 & \\
S2X35 & 18.1 & 8.3 & 44.4 & \\
S2E12 & 15.7 & 18.3 & 89.0 & \\
S2X16 & 13.7 & 13.1 & 89.1 & \\
S2H58 & 9.8 & 11.6 & 89.3 & \\
S2H13 & 19.4 & 20.4 & 87.8 & 84.3 \\
S2D106 & 10.7 & 6.2 & 89.0 & \\
S2X58 & 22.2 & 14.1 & 92.5 & \\
S2H14 & 9.1 & 8.9 & 98.6 & 95.5 \\
S2X227 & 9.4 & 8.5 & 93.3 & \\
\hline & & & & *blank = n.d.
\end{tabular}

e

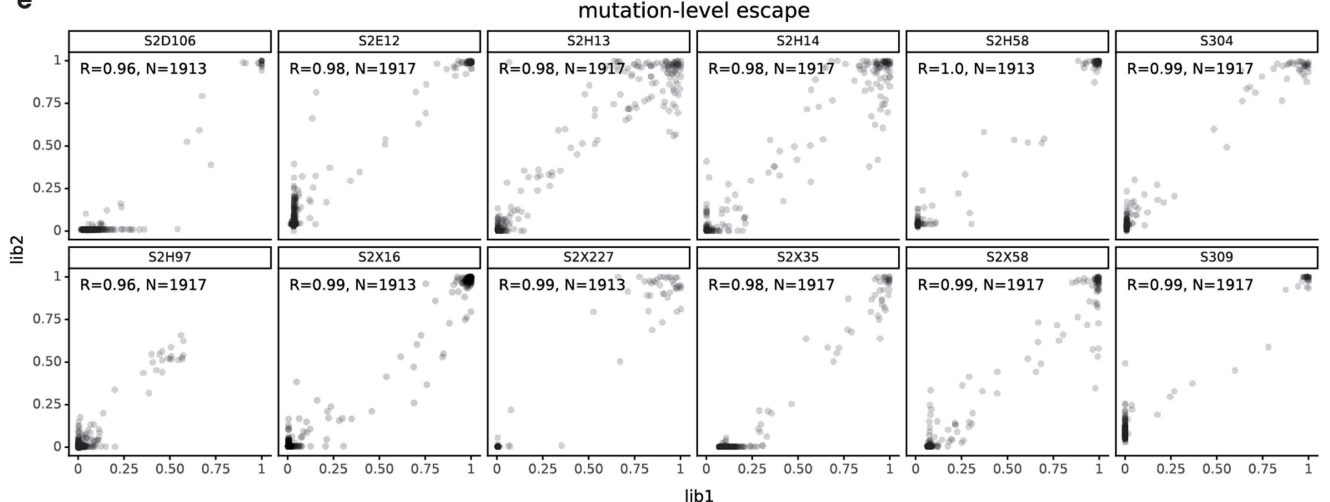

f

site-level escape

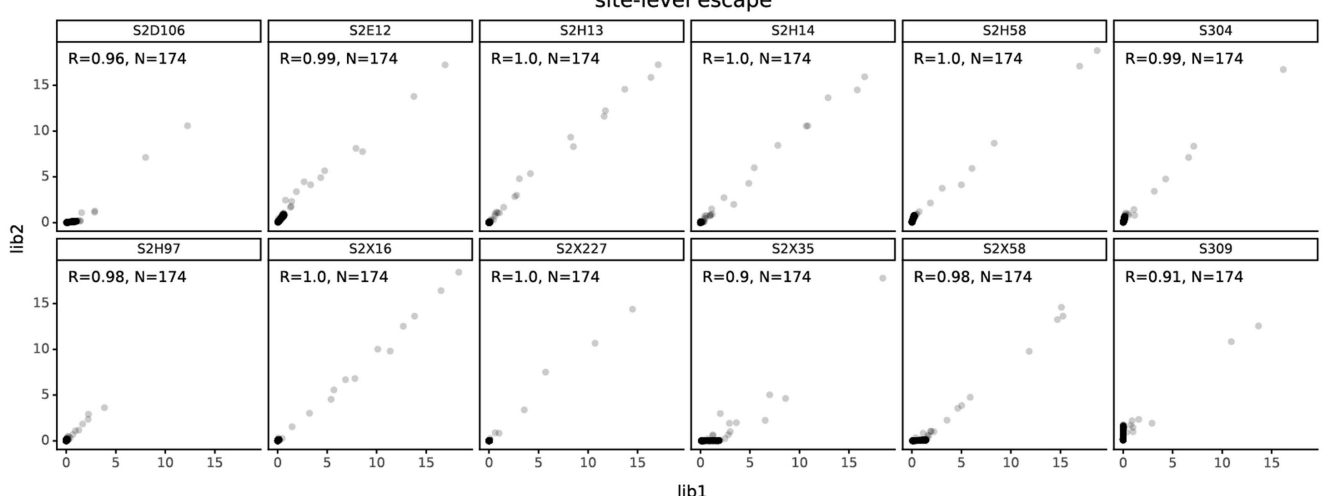

quantify variant frequency before and fter FACS via deep sequencing, compute escape fraction

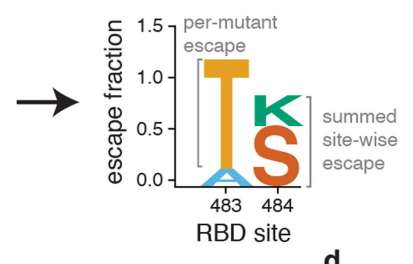

\section{d}
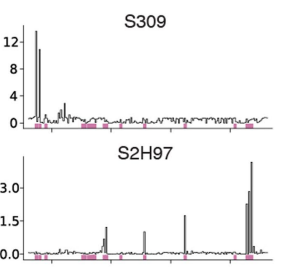

S304
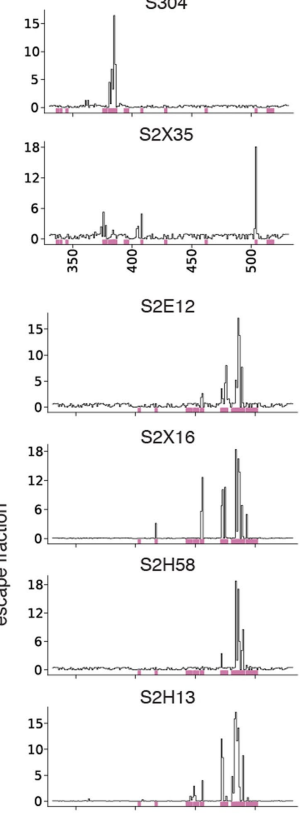

S2D106
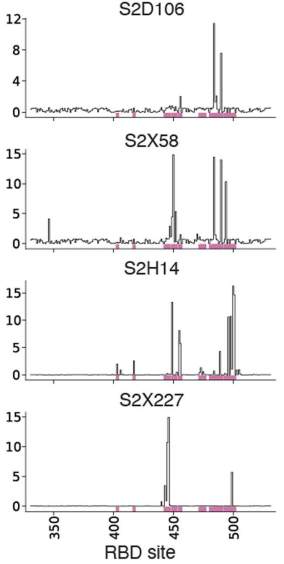

Extended Data Fig. 2 | See next page for caption. 
Extended Data Fig. 2 | Deep mutational scanning to map mutations that escape antibody binding. a, Scheme of the deep mutational scanning assay. Conformationally intact RBD is expressed on the surface of yeast, where RBD expression and antibody binding is detectable via fluorescent labelling. We previously constructed mutant libraries containing virtually all of the 3,819 possible amino acid mutations in the SARS-CoV-2 RBD ${ }^{26}$ and sorted the library to eliminate mutations that destabilize the RBD or strongly reduce ACE2 binding affinity ${ }^{3}$. We incubate the library with a sub-saturating antibody concentration and use fluorescence-activated cell sorting (FACS) to isolate yeast cells expressing RBD mutants with reduced antibody binding. Deep sequencing quantifies mutant frequencies before and after FACS selection, enabling calculation of the "escape fraction" of each amino acid mutation, which reflects the fraction of cells carrying that mutation that fall into the antibody-escape bin. Mutation escape fractions are represented in logo plots, where the height of a letter reflects the extent of escape from antibody binding. b, Representative selection gates, after gating for single cells expressing RBD as in Greaney et al. ${ }^{3}$. Yeast expressing the SARS-CoV-2 RBD (top panels) are labelled at $1 x, 0.01 x$ and no antibody to guide selection gates. Mutant RBDs that reduce binding (green, gate drawn to capture 0.01x wildtype control) are sorted and sequenced for calculation of mutant escape fractions. This same gate was used to quantify escape within libraries of yeast expressing all sarbecovirus RBD homologues. For several antibodies, we also selected the sarbecovirus RBD library with a more stringent "full escape" gate (blue, gate drawn to capture $0 \mathrm{ng} \mathrm{ml}^{-1}$ wild-type control).c, Fraction of library cells falling into escape bins for each antibody selection. d, Line plots showing total escape at all RBD sites for each antibody. Sites of strong escape illustrated in logo plots in Fig. 1b, c shown with pink indicators. e, f, Correlation in permutation (e) and per-site (f, sum of per-mutation) escape fractions for duplicate libraries that were independently generated and assayed. $N$, number of mutations (e) or sites (f) in the correlation. 

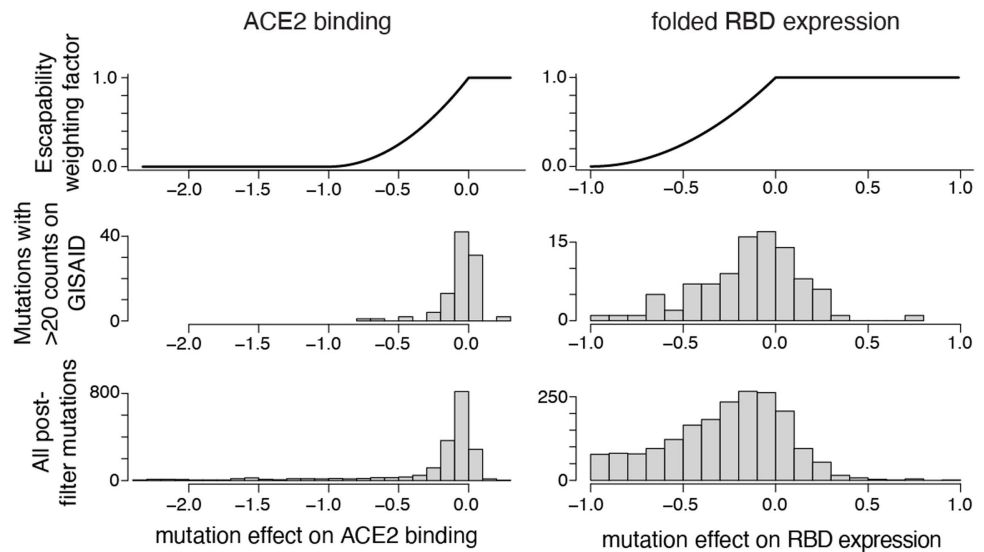
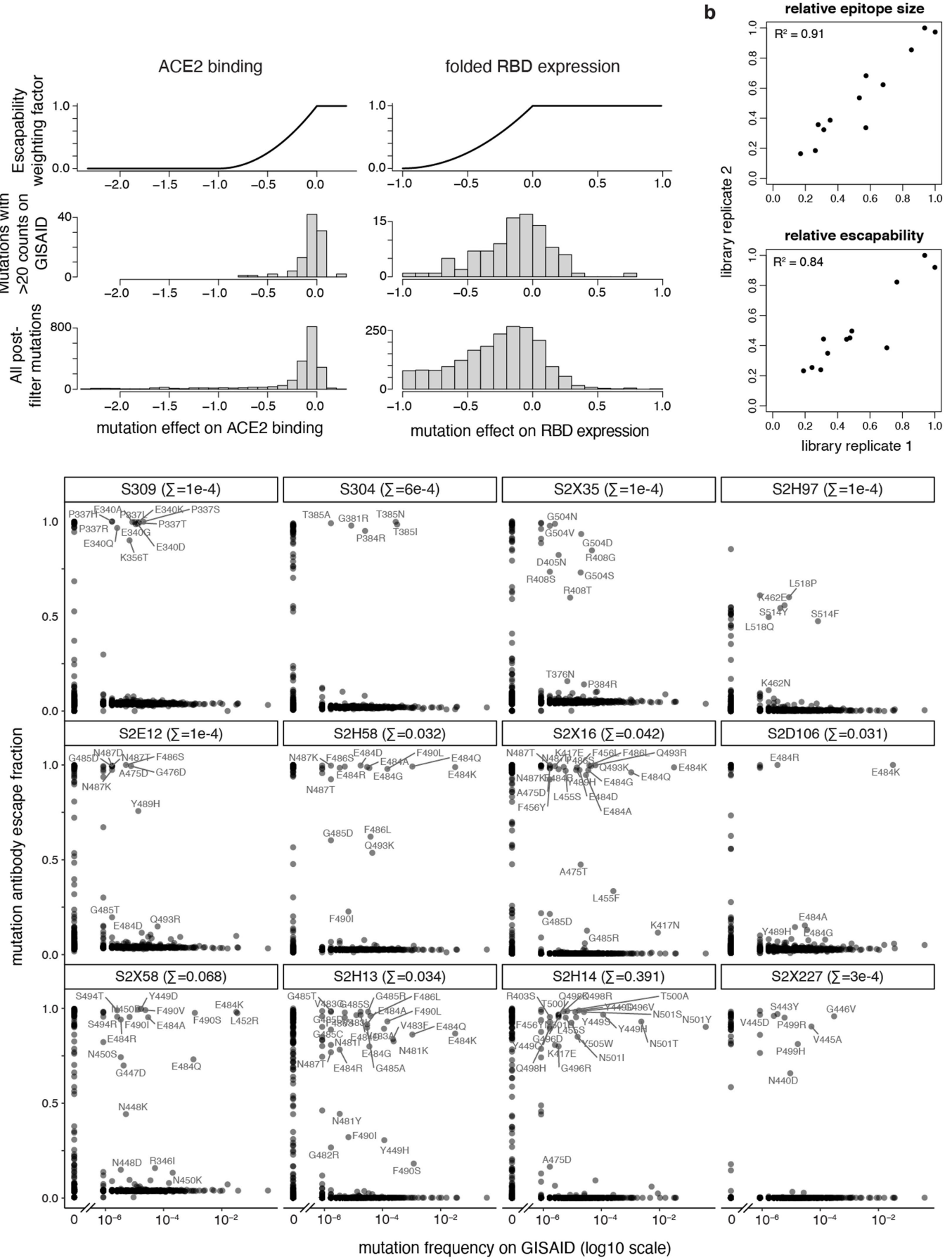

Extended Data Fig. 3 | Antibody escapability from deep mutational scanning measurements and in natural SARS-CoV-2 mutants. a, To calculate antibody escapability (Fig. 1b, c), mutation escape fractions were weighted by their deleterious consequences for ACE2 binding or RBD expression. Top plots show the weighting factor ( $y$-axis) for mutation effects on ACE2 binding (left) and RBD expression (right). This weight factor was multiplied by the mutation escape fraction in the summation to calculate antibody escapability as described in the Methods. Histograms show the distribution of mutation effects on ACE2 binding (left) and RBD expression (right) for all mutations that pass our computational filtering steps (bottom), and mutations that are found with at least 20 sequence counts on GISAID (middle). b, Correlation in antibody relative epitope size (top) and escapability (bottom) calculated from independent deep mutational scanning replicates, compared to the averaged replicates shown in Fig. $1 \mathrm{~b}, \mathrm{c} . \mathrm{R}^{2}$, squared Pearson correlation coefficient. c, Scatter plots illustrate the degree to which a mutation escapes antibody binding (escape fraction, $y$-axis) and its frequency among 1,190,241 high-quality human-derived SARS-CoV-2 sequences present on GISAID as of May 2, 2021. Large escape mutations (>5x global median escape fraction) for each antibody with non-zero mutant frequencies are labelled. Plot labels report the sum of mutant frequencies for all labelled mutations, corresponding to the natural SARS-CoV-2 mutant escape frequency for antibodies shown in Fig. 4d, g. 
a

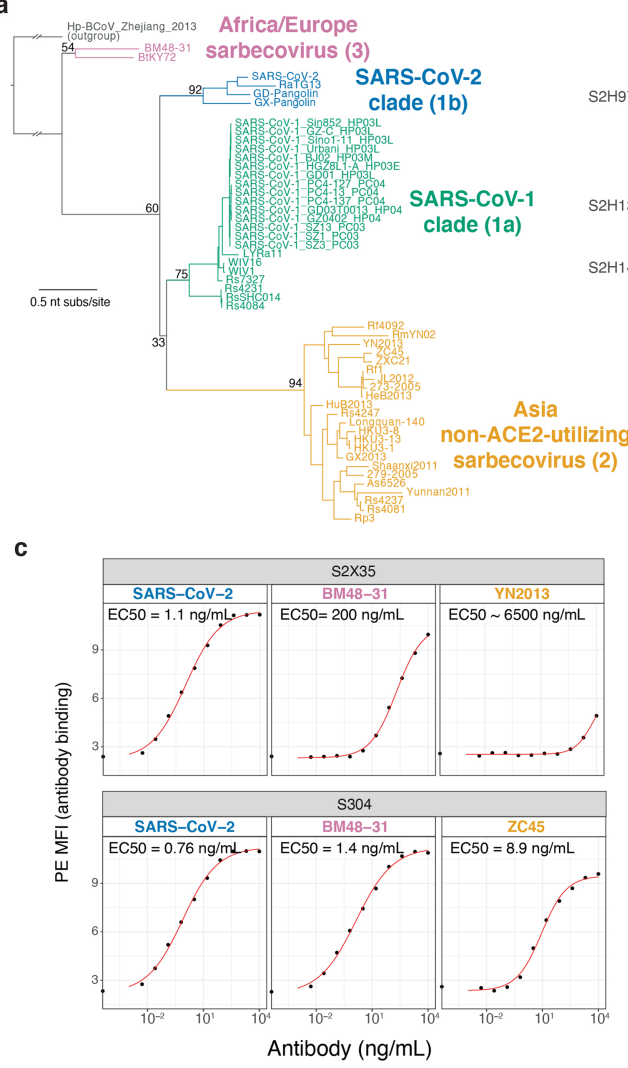

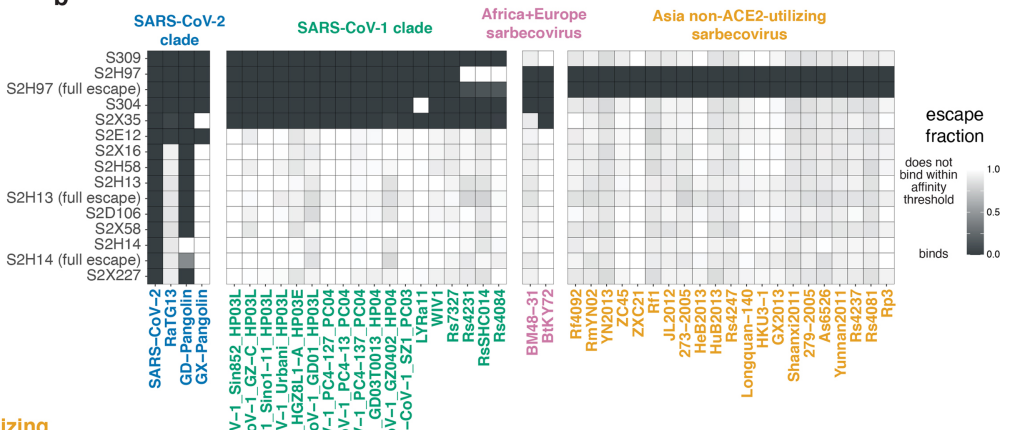

d
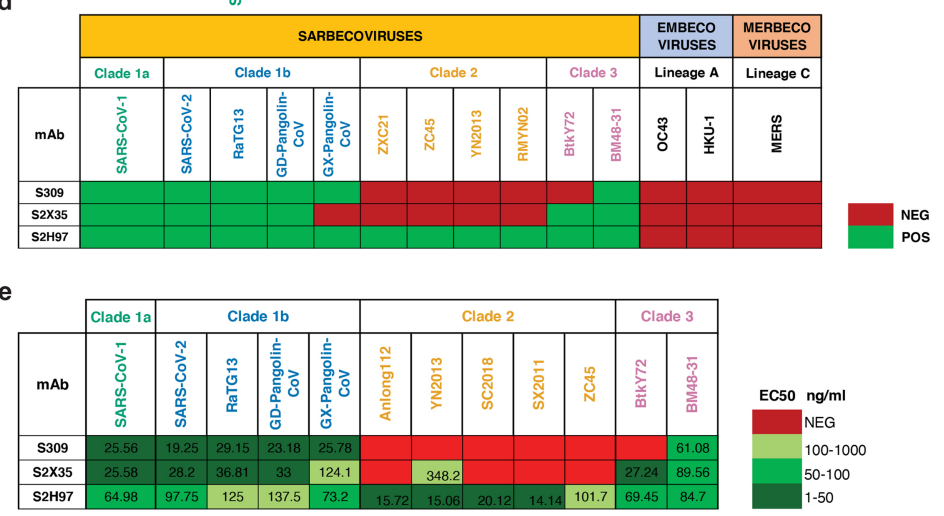

g

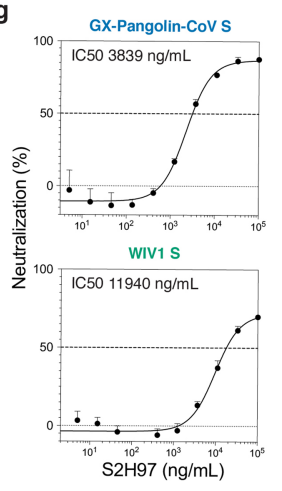

j

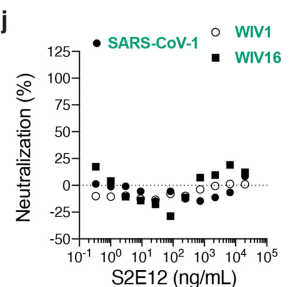

f $\mathrm{Fab} / \mathrm{RBD} \mathrm{K}_{\mathrm{D}}(\mathrm{nM})$

\begin{tabular}{|c|c|c|c|c|c|c|c|c|c|c|c|c|}
\hline \multirow{2}{*}{$\begin{array}{l}\text { Analyte } \\
\text { hACE2 }\end{array}$} & \multicolumn{4}{|c|}{ SARS-CoV-2 clade (1b) } & \multicolumn{2}{|c|}{$\begin{array}{l}\text { SARS-CoV-1 } \\
\text { clade (1a) }\end{array}$} & \multicolumn{2}{|c|}{$\begin{array}{l}\text { Africa/Europe } \\
\text { Clade (3) }\end{array}$} & \multicolumn{4}{|c|}{ Asia non-ACE2 Clade (2) } \\
\hline & $\begin{array}{c}\text { SARS } \\
58\end{array}$ & $\begin{array}{c}\text { RaTG13 } \\
\sim 9000\end{array}$ & $\begin{array}{l}\text { Pan-C } \\
27\end{array}$ & $\begin{array}{l}\text { Pan-GX } \\
32\end{array}$ & $\begin{array}{l}\text { SARS } \\
160\end{array}$ & $\begin{array}{c}\text { WIV1 } \\
93\end{array}$ & $\begin{array}{c}\text { BTKY72 } \\
\text { NB }\end{array}$ & $\begin{array}{c}\text { BM48-31 } \\
\text { NB }\end{array}$ & $\begin{array}{c}\text { ZC45 } \\
\text { NB }\end{array}$ & $\begin{array}{l}\text { YN201 } \\
\text { NT }\end{array}$ & $\begin{array}{r}\mathrm{SC}_{20} \\
\text { NT }\end{array}$ & $\begin{array}{l}\text { XX2011 } \\
\text { NT }\end{array}$ \\
\hline S & 0.2 & 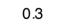 & 0.5 & 0 & 02 & 0.1 & $\mathrm{~N}$ & 140 & NB & $\mathrm{N}$ & NT & NT \\
\hline & 0.9 & 2. & 2.0 & 1.9 & 0.1 & 0.4 & 0.4 & 2.1 & 250 & v & 0 & 20 \\
\hline & 0.2 & 32 & 0.5 & $\sim 3000$ & 29 & 17 & 25 & $\sim 3000$ & NB & NT & T & NT \\
\hline & 1.8 & 110 & 1.3 & & $\mathrm{~N}$ & NB & NVD & & $\mathrm{NB}$ & 1 & $\sqrt{1}$ & NT \\
\hline $2 \mathrm{H} 97$ & 0.03 & 0.05 & 0.04 & 2.2 & 1.6 & 5.8 & 7.2 & 28 & 0.7 & 0.6 & 0.6 & 1.8 \\
\hline
\end{tabular}

h

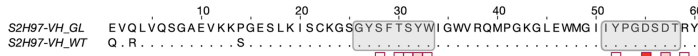

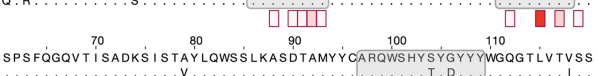
एiांए

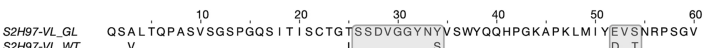

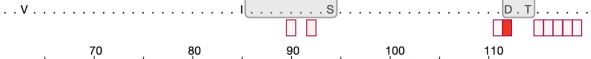

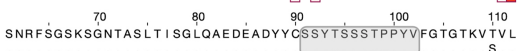

Paratope Binding Energy

k
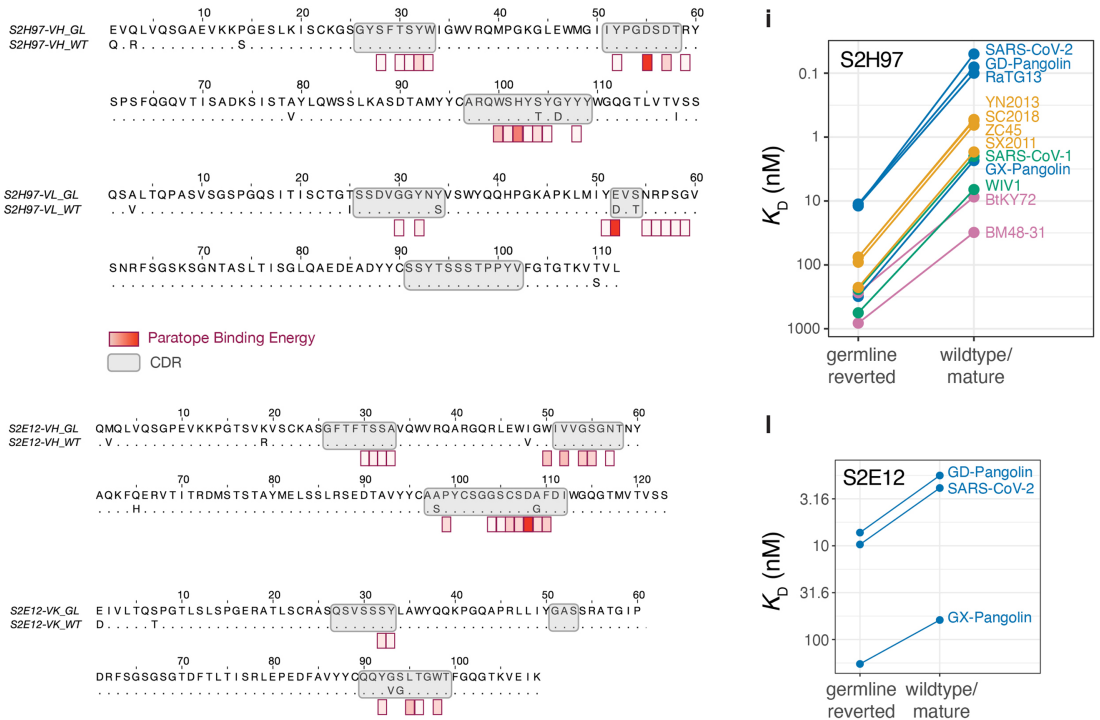

I

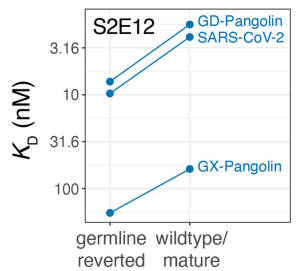

Extended Data Fig. 4 |See next page for caption. 


\section{Article}

Extended Data Fig. $4 \mid$ Breadth of antibody binding across sarbecoviruses. a, Phylogenetic relationship of sarbecovirus RBDs inferred from aligned nucleotide sequences, with the four sarbecovirus clades labelled in separate colours used throughout the text. Node support values are bootstrap support values. b, Breadth of sarbecovirus binding by each antibody to a panel of yeastdisplayed sarbecovirus RBDs. Data as in Fig. 1d, with the addition of secondary "full escape" selection data for S2H97, S2H13, and S2H14 $\left(0 \mathrm{ng} \mathrm{ml}^{-1}\right.$ wild-type control as shown in Extended Data Fig. 2b, c), enabling differentiation of RBDs with intermediate binding (for example, S2H97/RsSHC014) versus complete loss of binding. Escape fractions are calculated as the mean of replicate barcoded genotypes internal to the library. Median number of barcodes per RBD is 249 , with a range of 104 to 566 . The median SEM across escape fraction measurements is 0.019 , with a range of 0.00005 to 0.038 across all RBD/ antibody pairs. c, Flow cy tometry detection of antibody binding to isogenic yeast-displayed RBD variants. d, Flow cytometry detection of antibody binding to mammalian-surface displayed spikes. e, ELISA binding of antibody to purified RBD proteins. $f$, SPR measurement of binding of cross-reactive antibodies (Fab) and human ACE2 to select sarbecovirus RBDs. NB, no binding; $\mathrm{NT}$, not tested. Values from single replicates. $\mathrm{g}$, $\mathrm{S} 2 \mathrm{H} 97$ neutralization of VSV pseudotyped with select sarbecovirus spikes, with entry measured in ACE2transduced BHK-21 cells. Curves are representative of two independent experiments. Points represent means, error bars standard deviation from three technical replicates, and $\mathrm{IC}_{50}$ geometric mean of experiments. $\mathrm{IC}_{50}$ values are not comparable to other experiments on Vero E6 cells (for example, Fig. 2c) due to ACE2 overexpression and its impact on S2H97 neutralization.

h, Alignment of germline-reverted and mature S2H97 heavy- (top) and lightchain (bottom) amino acid sequences. CDR sequences shown in grey box. Heat map overlay indicates the predicted energetic contribution of antibody paratope residues from the crystal structure. $\mathbf{i}$, Binding of germline-reverted and mature $\mathrm{S} 2 \mathrm{H} 97$ to select sarbecovirus RBDs as measured by SPR.

j, Neutralization experiment of select sarbecoviruses by S2E12 (spikepseudotyped VSV on 293T-ACE2 cells). Details as in Fig. 3c. k, Alignment of germline-reverted and mature S2E12. Details as in h. I, Binding of germlinereverted and mature S2E12 to select sarbecovirus RBDs as measured by SPR. 


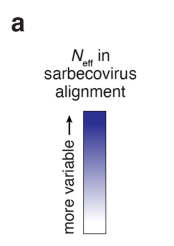

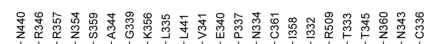

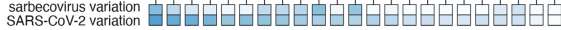
constraint ACEE aftinity
constrant, BBD olding

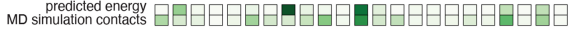

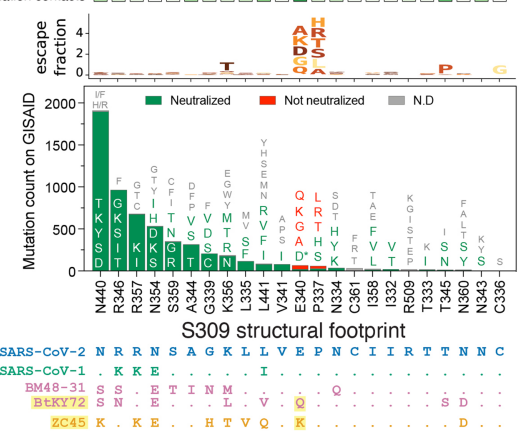

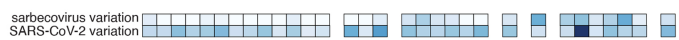

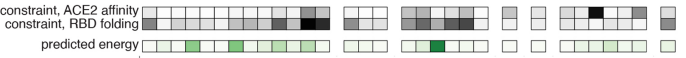

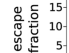

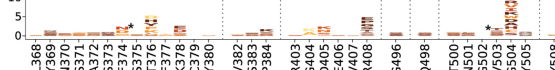
S2X35 structural footprint

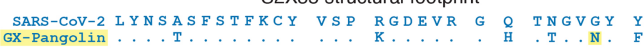

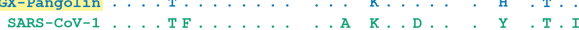

BM48-3

ZC45
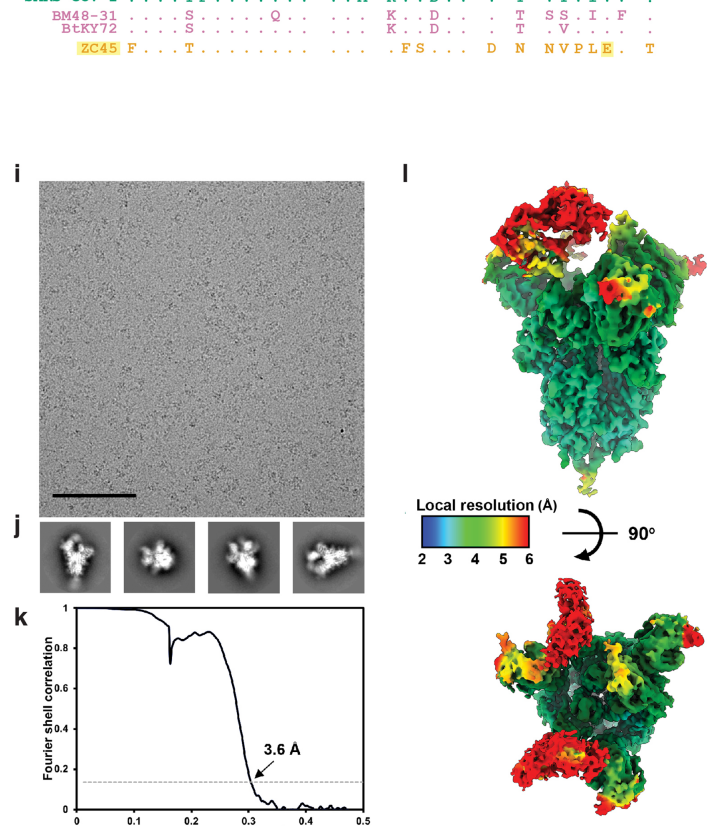

d

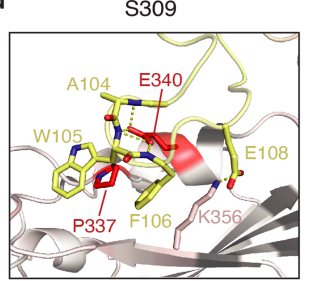

f

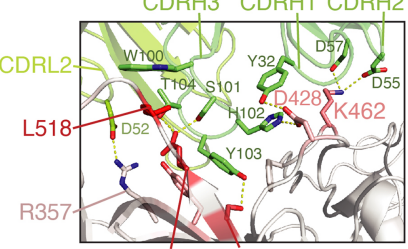

E516 S514

g

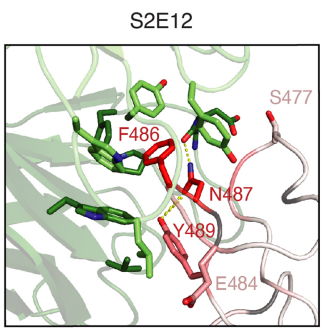

S2X35
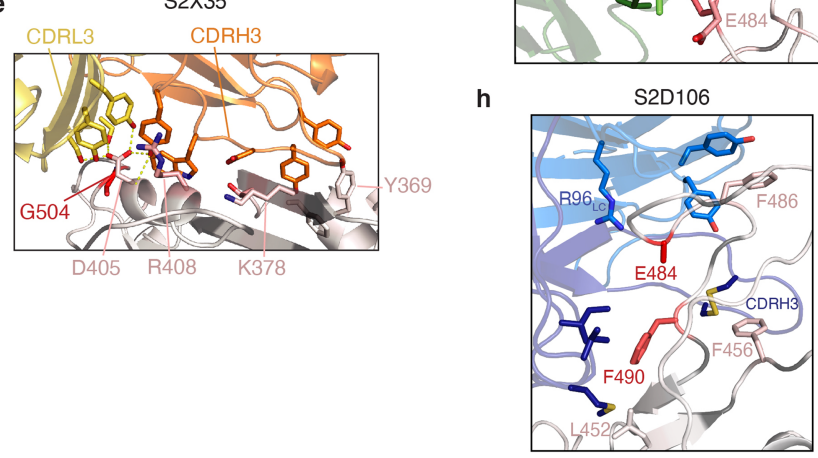

m

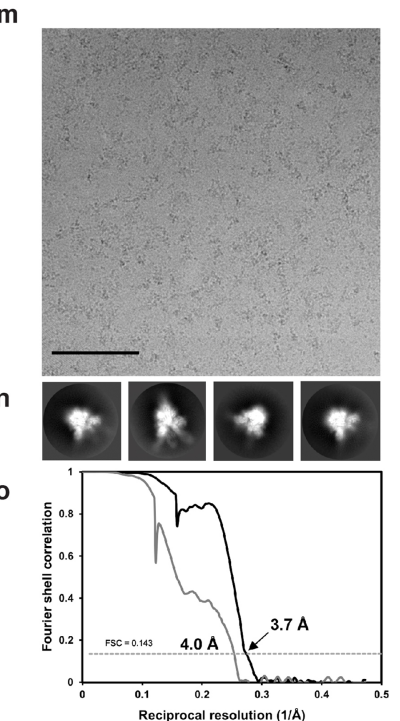

p

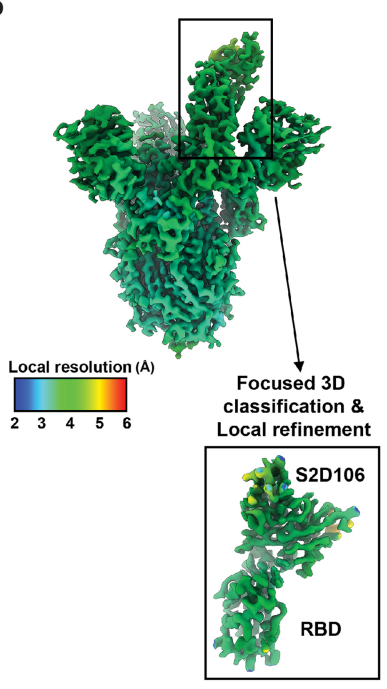

Extended Data Fig. 5 | See next page for caption. 


\section{Article}

Extended Data Fig. 5 | Structures and epitopes of Fab:RBD complexes.

a, Surfaces targeted by broadly binding RBD antibodies. RBD surface is coloured by site variability across sarbecoviruses. ACE 2 key motifs shown in transparent red cartoon. Antibody variable domains shown as cartoon, with darker shade indicating the heavy chain. b, c, Integrative features of the $\$ 309$ (b) and S2X35 (c) structural epitopes. Details as in Figs. 3g, h, 2b. Variants in the S309 epitope were tested with VIR-7831 with the exception of E340D (marked with *), which was tested with S309. d-h, Zoomed in view of the RBD bound to S309 (d), S2X35 (e), S2H97 (f), S2E12 (g), and S2D106 (h), with important contact and escape residues labelled. RBD residues coloured by total site escape [scale bar, right of (d)]. i, j, Representative electron micrograph and 2D class averages of SARS-CoV-2 $\mathrm{S}$ in complex with the S2H97 Fab embedded in vitreous ice. Scale bar: $400 \AA$ A Micrographs representative of 3138 micrographs. k, Gold-standard Fourier shell correlation curve for the S2H97bound SARS-CoV-2S trimer reconstruction. The 0.143 cut-off is indicated by a horizontal dashed line. I, Local resolution map calculated using cryoSPARC for the whole reconstruction with two orthogonal orientations.

$\mathbf{m}, \mathbf{n}$, Representative electron micrograph and 2D class averages of SARS-CoV-2 S in complex with the S2D106 Fab embedded in vitreous ice. Scale bar: $400 \AA$. Micrographs representative of 2166 micrographs o, Gold-standard Fourier shell correlation curves for the S2D106-bound SARS-CoV-2S trimer (black line) and locally refined RBD/S2D106 variable domains (grey line). The 0.143 cut-off is indicated by a horizontal dashed line. p, Local resolution map calculated using cryoSPARC for the whole reconstruction and the locally refined RBD/ S2D106 variable domain region. 


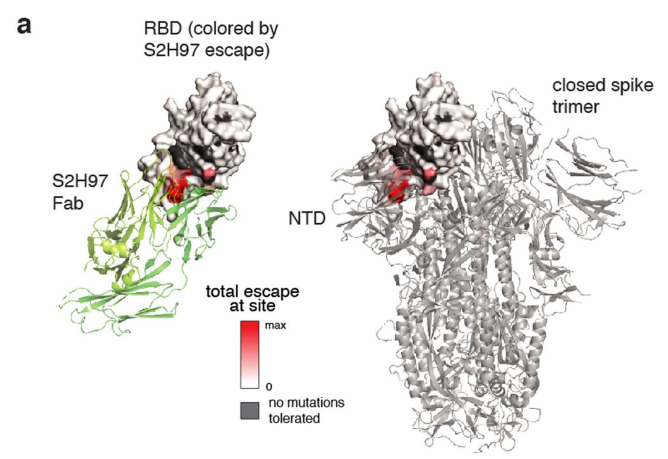

b

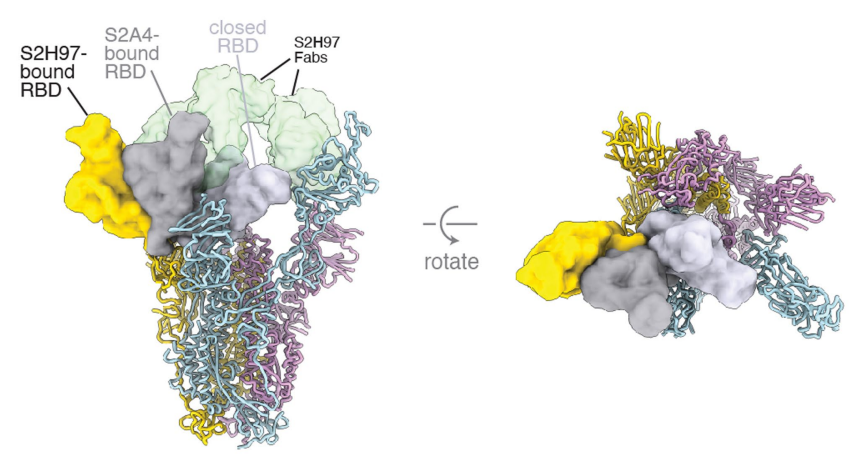

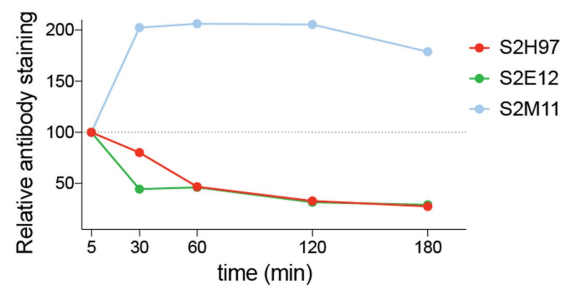

e

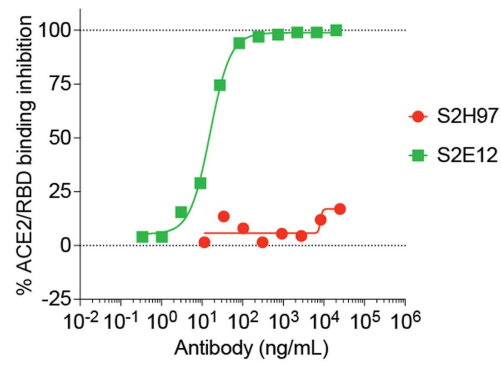

g

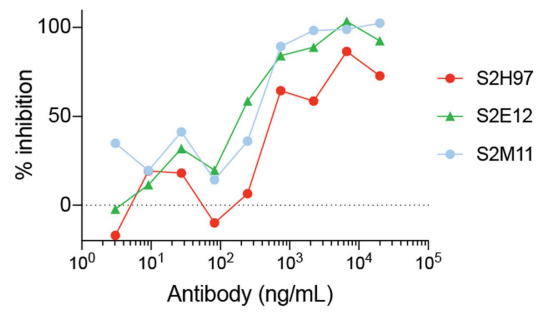

Extended Data Fig. 6 | Mechanism of action of S2H97 neutralization and protection.a, Quaternary context of the S2H97 epitope. Left, S2H97-bound RBD, with RBD sites coloured by S2H97 escape (scale bar, bottom). Right, RBD in the same angle as left, in the closed spike trimer. b, CryoEM structure of S2H97 Fabs (green surfaces) bound to SARS-CoV-2S indicating the extensive opening of the RBD (yellow surface) necessary to access the S2H97 epitope. Closed RBD (light purple surface, PDB 7K43) and site II Fab S2A4 bound open RBD (grey surface, PDB 7JVC) are shown for comparison. Spike protomers are shown in yellow, blue, and pink. c, Antibody-mediated S1 shedding from cell-surface expressed SARS-CoV-2S as determined by flow cytometry. d, Cell-cell fusion of $\mathrm{CHO}$ cells expressing SARS-CoV-2 S (CHO-S) incubated with variable concentrations of antibody. e, Antibody competition with RBD-ACE2 binding determined by ELISA. Points represent mean of technical duplicates.f, S2H97 neutralization of SARS-CoV-2 S pseudotyped VSV on d

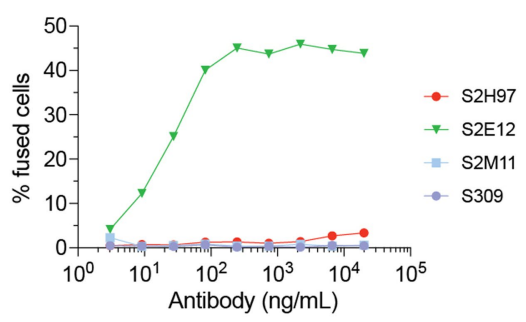

$\mathbf{f}$

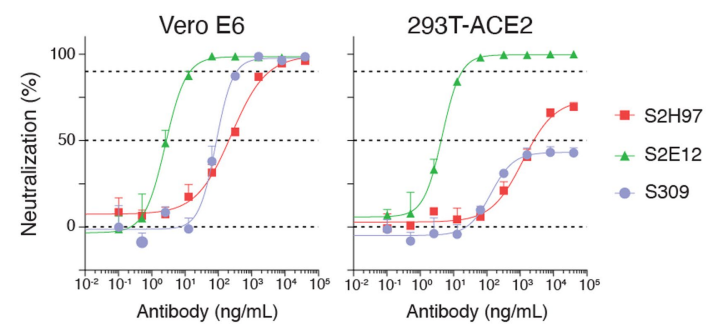

h

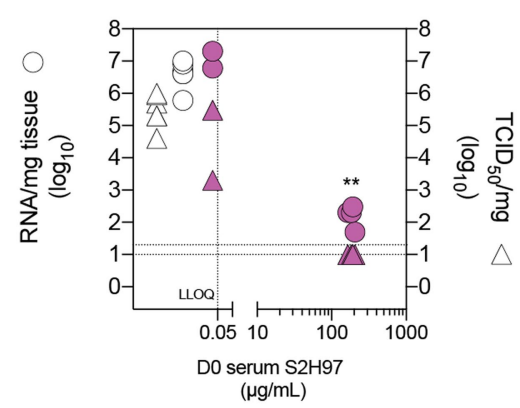

ACE2-overexpressing cells (293T-ACE2) compared to Vero E6 cells. Points reflect mean and error bars reflect standard deviation from triplicate measurements. Curves are representative of two biological replicates. g, Antibody inhibition of cell-to-cell fusion of Vero E6 cells transfected with SARS-CoV-2S. h, Influence of circulating S2H97 level on prophylactic efficacy in Syrian hamsters. Infectious virus titres (right $y$-axis, triangles) and RNA levels (left $y$-axis, circles) reflect the data represented in Fig. $2 \mathrm{f}$, measured in hamsters four days after SARS-CoV-2 challenge in animals prophylactically dosed with $25 \mathrm{mg} / \mathrm{kg} \mathrm{S} 2 \mathrm{H} 97$ (magenta symbols) or isotype control (white symbols). The levels of circulating S2H97 (D0, before infection, $\mu \mathrm{g} \mathrm{ml}^{-1}$ ) are shown on the $x$-axis (LLOQ, lower level of quantification). ${ }^{* *} P=0.0048$ (virus titre) and $P=0.0048$ (RNA) vs control isotype, two-sided Mann-Whitney test (the 2 animals shown with no detectable serum antibody were excluded from the comparison). 


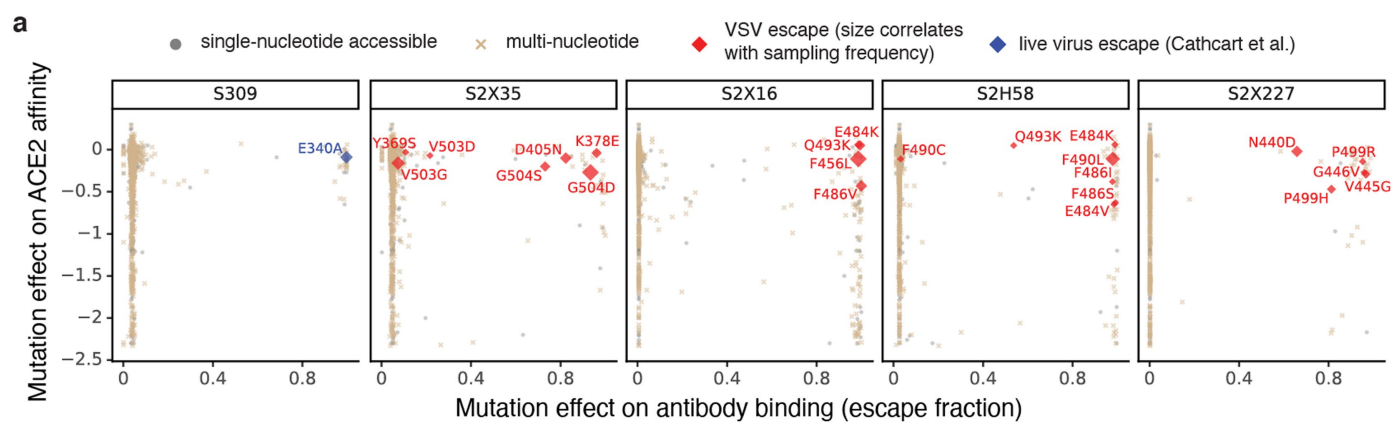

C

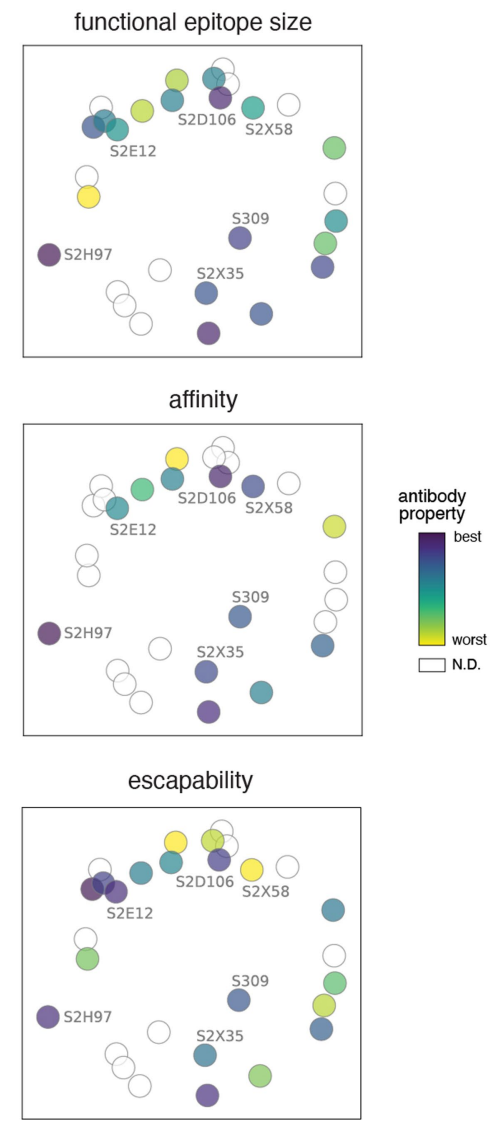

Extended Data Fig. 7 |Escapability and the relationships among antibody properties. a, Additional spike-VSV viral escape selections, as in Fig. 3a, and an illustration of the authentic SARS-CoV-2 escape data for VIR-7832 (derived from S309) reported in Cathcart et al. ${ }^{22}$.b. Correlation between the number of unique mutations selected across viral escape selection experiments and antibody escapability as tabulated in Fig. 1 b, c, plus S2X259 ${ }^{37}$. c, Projected epitope space from Fig. 4 a annotated by antibody properties as in Fig. $4 \mathrm{~b}-\mathrm{d}$. For each property, antibodies are coloured such that purple reflects the most desirable antibody (scale bar, right; N.D., not determined): narrowest $\begin{array}{lllllllllllllllllllllll}10^{4} & 10^{3} & 10^{2} & 10^{1} & 10^{2} & 10^{1} & 10^{\circ} & 10^{-1} & 0.2 & 0.4 & 0.6 & 0.8 & 1.0 & 0.6 & 0.2 & 1.0 & 0.6 & 0.2 & 10^{-1} & 10^{-2} & 10^{-3} & 10^{-4}\end{array}$
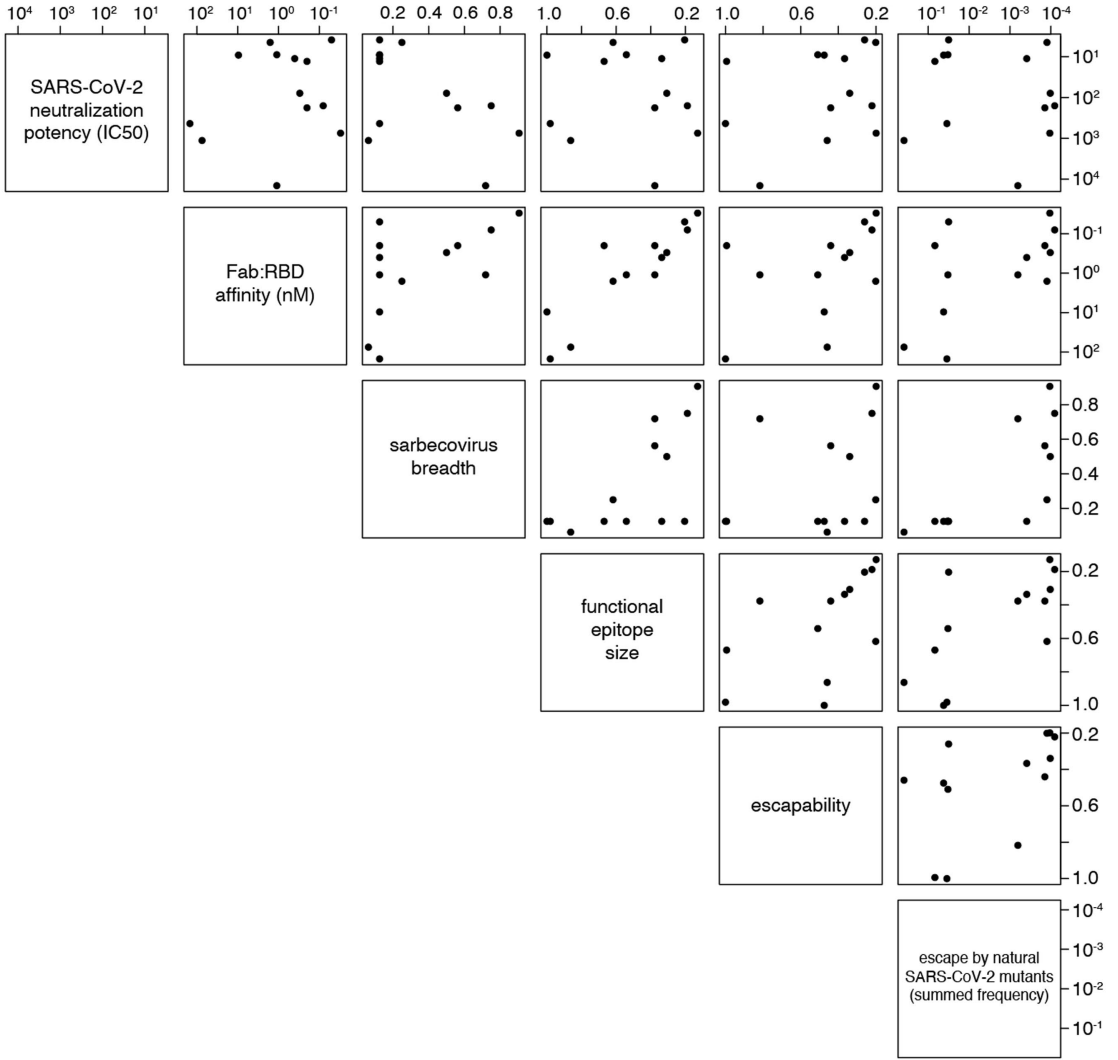

functional epitope, tightest binding affinity $\left(K_{\mathrm{D}}, \log 10\right.$ scale), lowest escapability. d, Pairwise scatter plots between all antibody properties discussed in the main text. Select scatter plots from this panel are shown in Figs. $4 \mathrm{e}-\mathrm{g}$. Details of each property described in Methods. All axes are oriented such that moving up on the $y$-axis and right on the $x$-axis corresponds to moving in the "preferred" direction for an antibody property (lower neutralization $\mathrm{IC}_{50}$, lower $K_{\mathrm{D}}$, higher breadth, narrower epitope size, lower escapability, lower total frequency of SARS-CoV-2 escape mutants among sequences on GISAID). 
Extended Data Table 1 | Characteristics of the antibodies described in this study

\begin{tabular}{|c|c|c|c|c|c|c|c|c|c|c|c|c|c|c|c|c|c|c|c|}
\hline \multirow[t]{2}{*}{$\begin{array}{l}\text { zo } \\
\text { w } \\
\bar{\sigma}\end{array}$} & \multirow[t]{2}{*}{$\stackrel{\mathscr{\varphi}}{\bar{\sigma}}$} & \multirow[t]{2}{*}{ 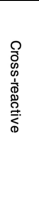 } & \multirow[t]{2}{*}{ 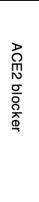 } & \multirow[t]{2}{*}{ 哇 } & \multirow[t]{2}{*}{ 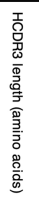 } & \multirow[t]{2}{*}{ 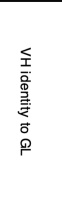 } & \multirow[t]{2}{*}{ 솛 } & $\begin{array}{l}\bar{\delta} \\
\text { 夏 } \\
\text { 害 }\end{array}$ & 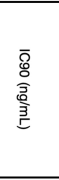 & 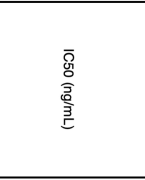 & 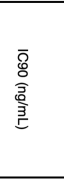 & 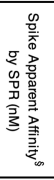 & \multicolumn{2}{|c|}{ 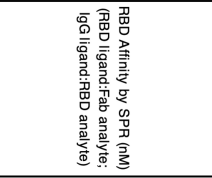 } & \multirow[t]{2}{*}{ 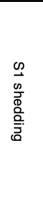 } & \multirow[t]{2}{*}{ 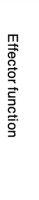 } & \multirow[t]{2}{*}{ 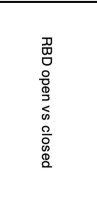 } & \multirow[t]{2}{*}{ 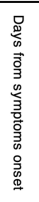 } & \multirow[t]{2}{*}{ 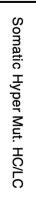 } \\
\hline & & & & & & & & \multicolumn{2}{|l|}{ VSV-pp } & \multicolumn{2}{|c|}{ SARS-CoV-2-Nluc } & Fab & Fab & $\lg G$ & & & & & \\
\hline S2E12 & la & No & Yes & VH1-58 & 16 & $97.2 \%$ & VK3-20 & $2.0(0.9-3.4)$ & 12.4 & $4.5(3-6)$ & 14.0 & $2.5^{\dagger}$ & $1.7(1.61-1.76)$ & 0.7 & Yes & No & open & 51 & $6 / 4$ \\
\hline S2X58 & Ib & No & Yes & VH1-46 & 12 & $99.0 \%$ & VK1-33 & $4.3(2.7-8.8)$ & 69.0 & $13.1(7-20)$ & 49.1 & 0.6 & $0.2(0.20-0.24)$ & 0.2 & Yes & No & open & 48 & $3 / 3$ \\
\hline $\mathrm{S} 2 \mathrm{H} 58$ & lb & No & Yes & $\mathrm{VH} 1-2$ & 15 & $97.9 \%$ & VK2-28 & $6.1(1.7-15.7)$ & 123.0 & $9.0(6-11)$ & 28.7 & 4.8 & $1.0(0.99-1.08)$ & 1.5 & No & Yes & ND & 45 & $6 / 3$ \\
\hline S2D106 & la & No & Yes & $\mathrm{VH} 1-69$ & 19 & $97.2 \%$ & VK1-39 & $4.3(3.5-6.5)$ & 25.6 & $6.8(5-9)$ & 23.6 & 0.17 & $0.05(0.046-0.049)$ & 0.04 & Yes & No & open & 98 & $5 / 3$ \\
\hline$S 2 \times 227$ & Ib & No & Yes & $\mathrm{VH} 1-46$ & 14 & $97.9 \%$ & VK4-1 & $5.3(4.4-6.2)$ & 26.9 & $11.2(9-13)$ & 36.0 & 0.11 & $0.4(0.41-0.46)$ & 0.1 & No & Yes & ND & 75 & $3 / 2$ \\
\hline $\mathrm{s} 2 \times 16$ & la & No & Yes & VH1-69 & 18 & $97.6 \%$ & VK2-28 & $8.1(2.6-14.9)$ & 62.1 & $9.2(4-16)$ & 39.7 & 11.1 & $9.2(8.83-9.59)$ & $6.1^{\prime \prime}$ & Yes & No & ND & 48 & $3 / 2$ \\
\hline S304 & Ilc & Yes & No & VH3-13 & 14 & $98.6 \%$ & VK1-39 & 9405 (8827-9795) & $\mathrm{n} / \mathrm{a}$ & 14412 (7573-29889) & $n / a$ & $8.7^{\star}$ & $1.0(0.93-1.17)$ & 0.4 & Yes & No & open & $10 y$ & $5 / 6$ \\
\hline s309 & IV & Yes & No & VH1-18 & 20 & $97.2 \%$ & VK3-20 & $31.1(23.0-58.7)$ & 160.9 & $78.9(52-121)$ & 300.3 & $\sim 0.2^{*}$ & $0.2(0.17-0.31)$ & $0.2^{\ddagger}$ & No & Yes & open/closed & $10 y$ & $8 / 6$ \\
\hline $\mathrm{s} 2 \times 35$ & $\mathrm{Ila}$ & Yes & Yes & VH1-18 & 21 & $98.6 \%$ & VL1-40 & $96.2(67.8-144.9)$ & 530.5 & $179.4(121-219)$ & 592.5 & $\sim 0.1^{*}$ & $0.2(0.18-0.22)$ & 0.06 & Yes & No & open & 48 & 4/1 \\
\hline S2H97 & $\mathrm{v}$ & Yes & No & VH5-51 & 13 & $98.3 \%$ & VL2-14 & $337.6(277-406)$ & 3656.1 & $748.7(540-1120)$ & 3290.7 & $\sim 2$ & $0.04(0.031-0.060)$ & 0.04 & Yes & No & open & 81 & $7 / 6$ \\
\hline $\mathrm{S} 2 \mathrm{H} 13$ & lb & No & Yes & VH3-7 & 13 & $97.9 \%$ & VL7-46 & $637.6(494-856)$ & 6745.9 & $435.3(331-656)$ & 1390.1 & $119^{*}$ & 123 (108-149) & $33^{\prime \prime}$ & No & Yes & open/closed & 17 & $4 / 1$ \\
\hline $\mathrm{S} 2 \mathrm{H} 14$ & la & No & Yes & $\mathrm{VH} 3-15$ & 17 & $100.0 \%$ & VL6-57 & $1274.0(1027-1436)$ & 8735.3 & $1130.3(522-2583)$ & 5121.8 & $90.1^{*}$ & $71(56-77)$ & $65^{\prime \prime}$ & Yes & No & open & 17 & $0 / 0$ \\
\hline
\end{tabular}

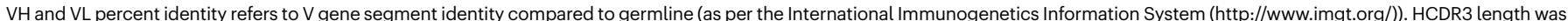
determined using IMGT. SARS-CoV-2 neutralization potency (authentic virus [ $n=3$ ] and spike-pseudotyped VSV particles [ $n=3$ to 8 ] on Vero E6 cells), Fab:RBD binding affinities measured by

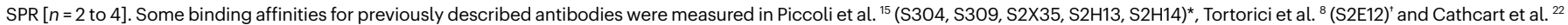
(VIR-7831, derived from S309). Values in brackets are minimum and maximum determined values. ${ }^{\S}$ Spike binding data are "apparent affinity" or $\mathrm{K}_{\mathrm{D}, \mathrm{app}}$, because RBD conformational dynamics

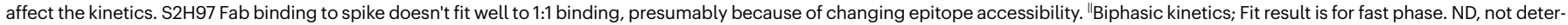
mined. 


\section{Article}

Extended Data Table 2 | Crystallographic data collection and refinement statistics

\begin{tabular}{|c|c|c|c|}
\hline & $\begin{array}{l}\text { RBD/S2X35/S309 } \\
\text { PDB 7R6W }\end{array}$ & $\begin{array}{l}\text { RBD/S2H97/S2X259 } \\
\text { PDB 7M7W }\end{array}$ & $\begin{array}{l}\text { RBD/S2E12/S309/S304 } \\
\text { PDB 7R6X }\end{array}$ \\
\hline \multicolumn{4}{|l|}{ Data collection } \\
\hline Space group & $\mathrm{C} 222$ & $\mathrm{P} 2_{1}$ & $\mathrm{I} 4{ }_{1} 22$ \\
\hline \multicolumn{4}{|l|}{ Cell dimensions } \\
\hline$a, b, c(\AA)$ & $106.27,239.37,129.81$ & $86.19,66.40,237.66$ & $245.87,245.87,237.31$ \\
\hline$\alpha, \beta, \gamma\left(^{\circ}\right)$ & $90.00,90.00,90.00$ & $90.00,94.34,90.00$ & $90.00,90.00,90.00$ \\
\hline Resolution $(\AA)$ & $39.52-1.83(1.86-1.83)$ & $63.94-2.65(2.70-2.65)$ & $49.00-2.93(2.99-2.93)$ \\
\hline$R_{\text {merge }}$ & $0.085(2.96)$ & $0.149 / 2.494$ & $0.295 / 7.868$ \\
\hline$I / \sigma I$ & $16.2(0.7)$ & $10.9(0.8)$ & $13.3(0.5)$ \\
\hline Completeness (\%) & $99.6(99.5)$ & $98.6(98.3)$ & $100.00(100.00)$ \\
\hline Redundancy & $6.7(7.0)$ & $6.9(6.8)$ & $28.9(27.2)$ \\
\hline \multicolumn{4}{|l|}{ Refinement } \\
\hline Resolution $(\AA)$ & 1.83 & 2.65 & 2.95 \\
\hline No. reflections & 135,667 & 73,189 & 71,532 \\
\hline$R_{\text {work }} / R_{\text {free }}$ & $0.211 / 0.232$ & $0.221 / 0.271$ & $0.232 / 0.262$ \\
\hline \multicolumn{4}{|l|}{ No. atoms } \\
\hline Protein & 8160 & 16,162 & 9,101 \\
\hline Ligand/ion & 172 & 28 & 16 \\
\hline Water & 584 & 95 & 3 \\
\hline \multicolumn{4}{|l|}{$B$-factors } \\
\hline Protein & 39.56 & 75.86 & 116.53 \\
\hline Ligand/ion & 75.00 & 84.00 & 122.00 \\
\hline Water & 42.94 & 50.09 & 65.90 \\
\hline \multicolumn{4}{|l|}{ R.m.s. deviations } \\
\hline Bond lengths $(\AA)$ & 0.004 & 0.002 & 0.003 \\
\hline Bond angles $\left(^{\circ}\right)$ & 1.259 & 0.817 & 0.936 \\
\hline
\end{tabular}

*Values in parentheses are for highest-resolution shell 
Extended Data Table 3 | CryoEM data collection, refinement, and validation statistics

\begin{tabular}{|c|c|c|c|}
\hline & $\begin{array}{l}\text { SARS-CoV-2 } \\
\text { S/S2D106 } \\
\text { (EMD-24300) }\end{array}$ & $\begin{array}{l}\text { SARS-CoV-2 } \\
\text { S/S2D106 } \\
\text { (local } \\
\text { refinement) } \\
\text { (EMD-24299) } \\
\text { (PDB 7R7N) } \\
\end{array}$ & $\begin{array}{l}\text { SARS-CoV-2 } \\
\text { S/S2H97 } \\
\text { (EMD-24301) }\end{array}$ \\
\hline \multicolumn{4}{|l|}{ Data collection and processing } \\
\hline Magnification & 130,000 & 130,000 & 36,000 \\
\hline Voltage (kV) & 300 & 300 & 200 \\
\hline Electron exposure $\left(\mathrm{e}-/ \AA^{2}\right)$ & 70 & 70 & 60 \\
\hline Defocus range $(\mu \mathrm{m})$ & $-0.5--2.5$ & $-0.5--2.5$ & $-0.5--3.0$ \\
\hline Pixel size $(\AA ̊)$ & 0.525 & 0.525 & 1.16 \\
\hline Symmetry imposed & $\mathrm{C} 1$ & $\mathrm{C} 1$ & $\mathrm{C} 1$ \\
\hline Initial particle images (no.) & 175,479 & 87,587 & 98,950 \\
\hline Map resolution $(\AA)$ & 3.7 & 4.0 & 3.6 \\
\hline FSC threshold & 0.143 & 0.143 & 0.143 \\
\hline Map sharpening $B$ factor $\left(\AA^{2}\right)$ & -61 & -17 & -70 \\
\hline \multicolumn{4}{|l|}{ Validation } \\
\hline MolProbity score & & 0.89 & \\
\hline Clashscore & & 0.58 & \\
\hline Poor rotamers $(\%)$ & & 0.45 & \\
\hline \multicolumn{4}{|l|}{ Ramachandran plot } \\
\hline Favored (\%) & & 96.78 & \\
\hline Allowed (\%) & & 3.22 & \\
\hline Disallowed (\%) & & 0 & \\
\hline
\end{tabular}




\section{Reporting Summary}

Nature Research wishes to improve the reproducibility of the work that we publish. This form provides structure for consistency and transparency in reporting. For further information on Nature Research policies, see our Editorial Policies and the Editorial Policy Checklist.

\section{Statistics}

For all statistical analyses, confirm that the following items are present in the figure legend, table legend, main text, or Methods section.

n/a Confirmed

$\square$ The exact sample size $(n)$ for each experimental group/condition, given as a discrete number and unit of measurement

$\square$ \ A statement on whether measurements were taken from distinct samples or whether the same sample was measured repeatedly

The statistical test(s) used AND whether they are one- or two-sided

$\square$ Only common tests should be described solely by name; describe more complex techniques in the Methods section.

Х $\square$ A description of all covariates tested

Х $\square$ A description of any assumptions or corrections, such as tests of normality and adjustment for multiple comparisons

$\searrow$ A full description of the statistical parameters including central tendency (e.g. means) or other basic estimates (e.g. regression coefficient)

$\bigotimes$ AND variation (e.g. standard deviation) or associated estimates of uncertainty (e.g. confidence intervals)

$\varnothing$ For null hypothesis testing, the test statistic (e.g. $F, t, r$ ) with confidence intervals, effect sizes, degrees of freedom and $P$ value noted Give $P$ values as exact values whenever suitable.

\ $\square$ For Bayesian analysis, information on the choice of priors and Markov chain Monte Carlo settings

Х $\square$ For hierarchical and complex designs, identification of the appropriate level for tests and full reporting of outcomes

Х $\square$ Estimates of effect sizes (e.g. Cohen's $d$, Pearson's $r$ ), indicating how they were calculated

Our web collection on statistics for biologists contains articles on many of the points above.

\section{Software and code}

Policy information about availability of computer code

\section{Data collection As described in Methods:}

* SPR binding data collected and analyzed using Biacore T200 Evaluation Software (v. 3.1) and Biacore Insight Evaluation Software (v. 2.0.15)

* FACSDiva software (version 6.1.3) for FACS data collection and cell sorting

* Leginon software (version 3.4) for electron microscopy data collection

* OpenMM (version 7.4.2) and Folding@home (core 22 0.0.13) for molecular dynamics simulation

* protein chromatography performed with UNICORN software (version 5.11)

* G*Power 3.1 for statistical power calculations

Data analysis * Deep mutational scanning data was analyzed using custom code that is available on GitHub, specifically: Repository containing all code, analysis, and summary notebooks for the analysis of the SARS-CoV-2 deep mutational scanning escape selections available on GitHub: https:// github.com/jbloomlab/SARS-CoV-2-RBD_MAP_Vir_mAbs. Repository containing code and analysis of the sarbecovirus RBD library binding experiments available on GitHub: https://github.com/jbloomlab/SARSr-CoV_RBD_MAP

* Neutralization assays, ELISA, cell biology, and serology assays analyzed using PRISM (versions 8.4.3 and 9.0.1) and Excel (version 16.45) as described in Methods.

* CryoEM data analysis performed with Warp (v. 1.09), Relion (v. 3.1) and CryoSPARC (version 3.2)

* Crystallography data analysis performed with XDS (VERSION Jan 31, 2020 BUILT=20200417), Coot (v. 0.9.5), ChimeraX (v. 1.1)/ISOLDE (v.

1.1), Refmac5 (v. 5.8.0267), and MOE (v. 2019.0102)

* Phylogenetic analysis performed with RAxML (version 8.2.12) and sequence alignment with MAFFT (version 7)

* MD simulation analyzed with custom code, available on GitHub: https://github.com/choderalab/rbd-ab-contact-analysis 
Policy information about availability of data

All manuscripts must include a data availability statement. This statement should provide the following information, where applicable:

- Accession codes, unique identifiers, or web links for publicly available datasets

- A list of figures that have associated raw data

- A description of any restrictions on data availability

* Raw Illumina sequencing data from deep mutational scanning experiments are available on NCBI SRA, BioSample SAMN18315604 (SARS-CoV-2 mutant selection data) and BioSample SAMN18316011 (sarbecovirus RBD selection data).

* PacBio sequencing data used to link N16 barcodes to sarbecovirus RBD variant are available on NCBI SRA, BioSample SAMN18316101.

* Complete table of deep mutational scanning antibody escape fractions is provided on GitHub: https://github.com/jbloomlab/SARS-CoV-2-RBD MAP Vir mAbs/ blob/main/results/supp_data/all_antibodies_raw_data.csv. This table includes both antibodies first described in this study (Fig. 1b,c), and all other antibody selections that were re-processed to generate Fig. 4a.

* The X-ray structure data and model has been deposited with accession code PDB 7R6W for RBD-S2X35-S309, PDB 7M7W for RBD-S2H97-S2X259 and PDB 7R6X for RBD-S2E12-S304-S309.

* CryoEM structure data and model are available with accession codes EMD-24300 for S/S2D106, EMD-24299 and PDB 7R7N for the S/S2D106 local refinement, and EMD-24301 for S/S2H97

* The raw and processed molecular dynamics trajectory data are available at the MoISSI COVID-19 Molecular Structure and Therapeutics Hub: https:// covid.molssi.org//simulations/\#foldinghome-simulations-of-the-sars-cov-2-spike-rbd-bound-to-monoclonal-antibody-s309 and https://covid.molssi.org// simulations/\#foldinghome-simulations-of-the-sars-cov-2-spike-rbd-bound-to-monoclonal-antibody-s2h97

* source data associated with hamster experiments in fig. $2 \mathrm{f}$ and extended data fig. $6 \mathrm{~g}$ are included as source data provided with the paper

\section{Field-specific reporting}

Please select the one below that is the best fit for your research. If you are not sure, read the appropriate sections before making your selection. \ Life sciences $\square$ Behavioural \& social sciences Ecological, evolutionary \& environmental sciences

For a reference copy of the document with all sections, see nature.com/documents/nr-reporting-summary-flat.pdf

\section{Life sciences study design}

All studies must disclose on these points even when the disclosure is negative.

Sample size For hamster prophylaxis experiments, sample size $=6$ was determined in order to have a significant difference of at least 1 log viral RNA (Effect Size $d=2.004$ ) between control and treated groups, by using a 2-tail T test with $80 \%$ power and an alpha error of 0.05 , calculated with G*Power 3.1 software

Data exclusions For S2H97 prophylaxis experiments in hamster, two hamsters in the $\mathrm{S} 2 \mathrm{H} 97$ arm were excluded from the statistical comparison. These hamsters had no detectable circulating antibody (LOD: $50 \mathrm{ng} / \mathrm{mL}$ ) at time of viral challenge (ELISA), which is a known technical failure in i.p. administration of hamster. The data justifying this exclusion in the statistical test is clearly shown in Extended Data Fig. 6h. No data were excluded from other experiments.

Replication Experimental assays were performed in biological duplicate or triplicate (or more) according to or exceeding standards in the field. Specifically, we perform deep mutational scans using two completely independently synthesized mutant libraries. We conducted all neutralization and antibody functional assays in biological duplicate, triplicate, or more, as indicated in relevant figure legends. In all cases, representative figure displays were appropriately replicated.

Randomization Hamsters were randomized to S2H97 and control treatment. For other experiments, randomization was not a relevant feature as we were applying a uniform set of techniques across a panel of candidate monoclonal antibodies

Blinding The hamster protection samples for RNA quantification and viral load titration were run by technicians who were blinded to treatment group of the analyzed samples

\section{Reporting for specific materials, systems and methods}

We require information from authors about some types of materials, experimental systems and methods used in many studies. Here, indicate whether each material, system or method listed is relevant to your study. If you are not sure if a list item applies to your research, read the appropriate section before selecting a response. 
Materials \& experimental systems

\begin{tabular}{l|l}
\hline$n / a$ & Involved in the study \\
$\square$ & $\bigotimes$ Antibodies \\
$\square$ Eukaryotic cell lines \\
$\square$ Palaeontology and archaeology \\
$\square$ & $\square$ Animals and other organisms \\
$\square$ & $\square$ Clinical data
\end{tabular}

Methods

$\mathrm{n} / \mathrm{a}$ Involved in the study

\ $\square$ chIP-seq

$\square \bigotimes$ Flow cytometry

\ $\square$ MRI-based neuroimaging

\section{Antibodies}

Antibodies used

* For antibody isolation, B cells were stained with CD19 PE-Cy7 (BD Biosciences 557835), anti-IgM (BioLegend 314508), anti-IgD (BD Bioscience 555779, anti-CD14 (BD Bioscience 562691), and anti-IgA (Southern Biotech 2050-09), and spike labeled with streptavidin Alexa-Fluor 647 (Life Technologies S21374)

* For FACS during deep mutational scanning, we used PE-conjugated goat anti-human-IgG (Jackson ImmunoResearch 109-115-098) and FITC-conjugated chicken anti-Myc (Immunology Consultants Lab, CYMC-45F).

* For VSV pseudotyped neutralization assays, media was supplemented with anti-VSV-G antibody (I1-mouse hybridoma supertant, ATCC CRL-2700)

* For SPR, immobilization was performed with anti-AviTag pAb (Genscript Cat \# A00674-40) and goat anti-human IgG Fc pAb, (Southern Biotech Cat \# 2014-01)

* For RBD ELISA, antibody binding was detected via goat anti-human IgG (Southern Biotech, 2040-04)

* For antibody blockade of ACE2 binding, RBD was detected via goat anti-mouse IgG (Southern Biotech 1030-04)

* For detection of circulating mAb levels in hamster experiments, an anti-human LS mutation antibody was custom-made by BioRad AbD Serotec, and anti-human $\mathrm{CH} 2$ antibody from BioRad cat\# MCA5748G clone R10Z8E9

* Monoclonal antibodies (S309, S2H97, S304, S2X35, S2E12, S2X16, S2H58, S2H13, S2D106, S2X58, S2H14, S2X227) were produced in-house using recombinant protein purification as described in the Methods. S2H97_GL and S2E12_GL were produced by ATUM.

Validation

We discovered and describe six new primary antibodies in this study. Target validation of the new antibodies was done with multiple binding assays (Extended Data Table 1), in addition to cryoEM and x-ray crystallography structures determined for two of the new antibodies. The other primary antibodies used in this study were reported previously in Pinto et al. Nature (doi: 10.1038/ s41586-020-2349-y.), Tortorici et al. Science (PMID doi: 10.1126/science.abe3354), and Piccoli et al. Cell (doi: 10.1016/ j.cell.2020.09.037).

Reactivity of primary antibodies listed above is based on the information on manufacturer's homepages.

\section{Eukaryotic cell lines}

Policy information about cell lines

Cell line source(s)

Cell lines were received from ATCC (Vero E6, Vero, BHK-21, CHO-K1), Takara (Lenti-X 293T) and Thermo Fisher Scientific (ExpiCHO-S, Expi293F and Freestyle 293-F). MA104 cells were a gift from Harry Greenberg. 293T-ACE2 cells are described in Ref 31 and Ref 43.

Authentication

No authentication was performed beyond manufacturer standards

Mycoplasma contamination

Vero and MA104 cell lines tested negative for mycoplasma contamination. Other cell lines were not tested.

Commonly misidentified lines (See ICLAC register)

No commonly misidentified cell lines were used in the study.

\section{Animals and other organisms}

Policy information about studies involving animals; ARRIVE guidelines recommended for reporting animal research

Laboratory animals

Experiments used Syrian hamsters (Mesocricetus auratus) from Janvier Laboratories. Female hamsters were used, at 6-10 week age

Wild animals

The study did not involve wild animals

Field-collected samples

The study did not involve field studies

Ethics oversight

Housing conditions and experimental procedures were approved by the ethical committee of animal experimentation of KU Leuven (license P065-2020). Experiments were performed in the high-containment A3 and BSL3+ facilities of the KU Leuven Rega Institute (3CAPS) under licenses AMV 30112018 SBB 21920180892 and AMV 23102017 SBB 21920170589 according to institutional guidelines.

Note that full information on the approval of the study protocol must also be provided in the manuscript. 
Policy information about studies involving human research participants

Population characteristics

Participants were identified by prior SARS-CoV-2 infection for PBMC isolation. Blood drawn from donor S2X was obtained at day 48 (S2X16, S2X35 and S2X58 mAbs) and 75 (S2X227) after symptoms onset. Blood from donor S2H was obtained at day 17 (S2H13 and S2H14), day 45 (S2H58) and day 81 (S2H97) after symptoms onset. Blood from donor S2D was obtained at day 98 (S2D106) after symptoms onset. Ages and sex for patients for mAb isolation: S2D: Age 70, male; S2H: Age 36, Male; S2X: Age 52, Male.

For vaccinated and convalescent cohorts used in serology assays, average age (and range) for was $52(25,78)$ and $49(28,69)$ for convalescent and vaccinated cohorts, respectively. Gender breakdown was $54.8 \%$ and $64.7 \%$ male for convalescent and vaccinated cohorts, respectively.

Recruitment

Patients were recruited on the basis of prior SARS-CoV-2 infection or vaccination in the hospital or outpatient setting. Patients were healthy volunteers who donated blood after being informed about the study. The only exclusion criteria used were HIV or other debilitating disease, but other information about diagnosis and treatment was not collected.

Ethics oversight

Study protocols for antibody isolation were approved by the local Institutional Review Board (Canton Ticino Ethics Committee, Switzerland), and all donors provided written informed consent for the use of blood and blood components. Study protocols for serological assays were approved by the local Institutional Review Boards relevant for each of three cohorts of samples (Canton Ticino Ethics Committee, Switzerland, the Ethical Committee of Luigi Sacco Hospital, Milan, Italy, and WCG North America, Princeton, NJ, USA). All donors provided written informed consent for the use of blood and blood components (such as PBMCs, sera or plasma) and were recruited at hospitals or as outpatients.

Note that full information on the approval of the study protocol must also be provided in the manuscript.

\section{Flow Cytometry}

Plots

Confirm that:

\The axis labels state the marker and fluorochrome used (e.g. CD4-FITC).

\The axis scales are clearly visible. Include numbers along axes only for bottom left plot of group (a 'group' is an analysis of identical markers).

\All plots are contour plots with outliers or pseudocolor plots.

\A numerical value for number of cells or percentage (with statistics) is provided.

\section{Methodology}

Sample preparation

Instrument

Software

Cell population abundance

Gating strategy

\ick this box to confirm that a figure exemplifying the gating strategy is provided in the Supplementary Information.
Yeast were induced for cell-surface expression, incubated with the primary antibody from the panel, and secondary labeled with anti-Myc and anti-Fc fluorescent conjugates as described in the Methods.

FACSAria II

FACSDiva Version 6.1.3

We work with homogenous yeast libraries in our deep mutational scanning experiments, therefore cell population abundance is not a detail of our approach.

Yeast expressing unmutated SARS-CoV-2 RBD are labeled at $1 x$ and $0.01 x$ the concentration used for library labeling. Gates are drawn on the basis of these controls to standardize selection stringency across expreriments. Gating schemes are shown in Extended Data Fig. 2 